\title{
THE NORTH AMERICAN AND PELICAN NEBULAE. II. MIPS OBSERVATIONS AND ANALYSIS
}

\author{
L. M. Rebull ${ }^{1}$, S. Guieu ${ }^{2}$, J. R. Stauffer ${ }^{1}$, L. A. Hillendrand ${ }^{3}$, A. Noriega-Crespo ${ }^{1}$, K. R. Stapelfeldt ${ }^{4}$, S. J. Carey ${ }^{1}$,

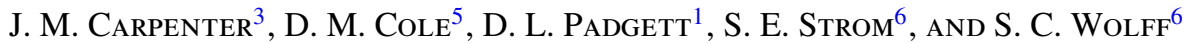 \\ ${ }^{1}$ Spitzer Science Center/Caltech, M/S 220-6, 1200 East California Boulevard, Pasadena, CA 91125, USA; luisa.rebull@jpl.nasa.gov \\ ${ }^{2}$ European Southern Observatory, Alonso de Cordova 3107, Vitacura, Santiago, Chile \\ ${ }^{3}$ Department of Astronomy, California Institute of Technology, Pasadena, CA 91125, USA \\ ${ }^{4}$ Jet Propulsion Laboratory, MS 183-900, California Institute of Technology, Pasadena, CA 91109, USA \\ ${ }^{5}$ Raytheon, Pasadena, CA, USA \\ ${ }^{6}$ NOAO, Tucson, AZ, USA \\ Received 2010 November 4; accepted 2011 February 1; published 2011 March 14
}

\begin{abstract}
We present observations of $\sim 7 \mathrm{deg}^{2}$ of the North American and Pelican Nebulae region at 24, 70, and $160 \mu \mathrm{m}$ with the Spitzer Space Telescope Multiband Imaging Photometer for Spitzer (MIPS). We incorporate the MIPS observations with earlier Spitzer Infrared Array Camera (IRAC) observations, as well as archival near-infrared (IR) and optical data. We use the MIPS data to identify 1286 young stellar object (YSO) candidates. IRAC data alone can identify 806 more YSO candidates, for a total of 2076 YSO candidates. Prior to the Spitzer observations, there were only 200 YSOs known in this region. Three subregions within the complex are highlighted as clusters: the Gulf of Mexico, the Pelican, and the Pelican's Hat. The Gulf of Mexico cluster is subject to the highest extinction $\left(A_{V}\right.$ at least $\left.\sim 30\right)$ and has the widest range of infrared colors of the three clusters, including the largest excesses and by far the most point-source detections at $70 \mu \mathrm{m}$. Just $3 \%$ of the cluster members were previously identified; we have redefined this cluster as about 10-100 times larger (in projected area) than was previously realized.
\end{abstract}

Key words: circumstellar matter - infrared: ISM - infrared: stars - ISM: clouds - ISM: individual objects (NGC 7000, IC 5070, LDN 935) - stars: formation - stars: pre-main sequence

Online-only material: color figures, machine-readable tables

\section{INTRODUCTION}

Much of our current knowledge regarding star-forming patterns and circumstellar disk evolution is derived from the study of molecular cloud complexes within a few hundred parsecs of the Sun. Among this group are a large number of lower-mass clouds such as Taurus (140 pc; Torres et al. 2007), populated with $\sim 300$ low-mass $\left(<2 M_{\odot}\right.$ ) young stars (see, e.g., Kenyon et al. 2008). Also among them but more infrequent are dense clusters like the Orion Nebula Cluster (ONC; 400 pc; see, e.g., Menten et al. 2007 or Muench et al. 2008), the prototypical high-mass and high-density star-forming region. The nearby cloud complexes (e.g., Rebull et al. 2010; Megeath et al. 2005) have served as our primary empirical guide to understanding the formation and early evolution of stars. However, they provide snapshots of only certain kinds of star formation. Because environment appears to play a significant role in defining stellar properties such as stellar rotation rates (e.g., Clarke \& Bouvier 2000; Briggs et al. 2007), disk evolution timescales (e.g., Robberto et al. 2004), and multiplicity (e.g., Kraus \& Hillenbrand 2007), for a comprehensive understanding of star formation, it is important that we study more than just the nearest examples of star formation.

The North American (NGC 7000) and Pelican (IC 5070) Nebulae (the entire complex is referred to hereafter as "NAN") is a complicated mixed-mode star formation region. NGC 7000 and IC 5070 appear to lie on either side of Lynds Dark Nebula (LDN) 935, but all three (NGC 7000, IC 5070, L935) are generally regarded as part of the same large $\mathrm{H}$ II complex (see, e.g., Wendker et al. 1983). We take the distance to the NAN to be 520 pc based on Laugalys et al. (2006a) and Straižys et al. (1989), though Laugalys \& Straižys (2002) claim a distance of $600 \mathrm{pc}$; see distance discussion in Guieu et al. (2009). Our results here are not strongly dependent on the distance assumed to the complex.

The NAN has a mass in molecular gas of order $10^{5} M_{\odot}$ (Feldt $\&$ Wendker 1993). There are at least 50 Herbig AeBe stars scattered through the complex (e.g., Herbig 1958), at least nine embedded or partially embedded young clusters (Cambrésy et al. 2002), and a variety of other signposts of current star formation, such as $\mathrm{HH}$ objects, jets, FU Ori stars, and strong $\mathrm{H} \alpha$ emission line stars (e.g., Bally \& Reipurth 2003; Ogura et al. 2002; Comerón \& Pasquali 2005; Marcy 1980; see Reipurth \& Schneider 2008 for a review). The few hundred largely unobscured young stellar objects (YSOs) that have been previously identified as members of the complex likely range in age from $<1 \mathrm{Myr}$ to several Myr. If one extrapolates using a typical initial mass function from the already-identified high-mass NAN population, the predicted mass in stars (to $0.2 M_{\odot}$ ) should be at least of order $10^{4} M_{\odot}$ and, given the age of the region, there should be thousands of low-mass classical T Tauri and weak-lined T Tauri members of the complex. In addition to the unobscured population, an embedded population was suspected (e.g., Herbig 1958; Osterbrock 1957). Using more than a million Two Micron All-Sky Survey (2MASS) sources in a $2.5 \times 2.5$ region, Cambrésy et al. (2002) mapped extinction and reddening toward the NAN complex, finding visual extinction up to $\sim 30 \mathrm{mag}$, as well as embedded clusters. Thus, star formation is ongoing.

The line of sight to the NAN $(l, b=84.8,-0.53)$ is heavily populated with foreground and background stars; the contamination is estimated at $1900 \pm 500 \mathrm{stars}^{\mathrm{deg}} \mathrm{g}^{-2}$ to the depth of the 2MASS survey (Cambrésy et al. 2002). One way of identifying members of the NAN as distinct from the Galaxy is by looking for those that have the prominent infrared (IR) signatures indicative of circumstellar disks. This 
Table 1

Summary of MIPS Observations (from Programs 20015 and 462)

\begin{tabular}{|c|c|c|c|c|}
\hline Field & $\begin{array}{c}\text { Map Center } \\
\text { (R.A., decl. in sexagesimal) }\end{array}$ & $\begin{array}{c}\text { Map Center } \\
\text { (R.A., decl. in degrees) }\end{array}$ & $\begin{array}{c}\text { Map Offset } \\
\text { (cross-scan, arcsec) }\end{array}$ & AORKEY \\
\hline Map 1 & $20: 54: 18,+44: 05: 00$ & $313.575,44.0833$ & -3300 & 16793600 \\
\hline Map 2 & $20: 54: 18,+44: 05: 00$ & $313.575,44.0833$ & -2200 & 16793856 \\
\hline Map 3 & $20: 54: 18,+44: 05: 00$ & $313.575,44.0833$ & -1100 & 16794112 \\
\hline Map 4 & $20: 54: 18,+44: 05: 00$ & $313.575,44.0833$ & 0 & 16794368 \\
\hline Map 5 & $20: 54: 18,+44: 05: 00$ & $313.575,44.0833$ & +1100 & 16794624 \\
\hline Map 6 & $20: 54: 18,+44: 05: 00$ & $313.575,44.0833$ & +2200 & 16794880 \\
\hline Map 7 & $20: 54: 18,+44: 05: 00$ & $313.575,44.0833$ & +3300 & 16795136 \\
\hline Patch 1 & $21: 00: 52,+44: 32: 33$ & $313.217,44.5425$ & $\ldots$ & 24251136 \\
\hline Patch 2 & $21: 01: 56,+45: 00: 06$ & $315.483,45.0017$ & $\ldots$ & 24253440 \\
\hline
\end{tabular}

region of sky was in the few percent missed by the allsky survey conducted in 1983 by the Infrared Astronomical Satellite (IRAS), but it has been observed by both the Midcourse Space Experiment (MSX) and AKARI. Given the high source density and high contamination rate, both relatively high spatial resolution and relatively deep observations are crucial for extracting candidate cluster members. We have obtained deep Spitzer Space Telescope (Werner et al. 2004b) Infrared Array Camera (IRAC; Fazio et al. 2004) and Multiband Imaging Photometer for Spitzer (MIPS; Rieke et al. 2004) maps of a $\sim 7 \mathrm{deg}^{2}$ region covering most of the complex. This paper is the second in our series on the NAN complex; Guieu et al. (2009; hereafter G09) have presented the IRAC observations, and this paper presents the MIPS observations and analysis.

MIPS observations at 24,70 , and $160 \mu \mathrm{m}$ can elucidate many aspects of the ongoing star formation in the NAN. Although the emission from stellar photospheres is falling rapidly at $24 \mu \mathrm{m}$, emission from circumstellar material makes many of the young cluster members quite bright at $24 \mu \mathrm{m}$, so MIPS easily distinguishes the young stars from most of the background Galactic population. Diffuse emission from the cloud itself gives us the location of much of the dust and molecular material. This paper presents the NAN MIPS observations, calling out properties unique to the MIPS bands, and using data from other wavelengths as required in order to obtain a more complete list of YSO members. MIPS reveals complex extended emission throughout the NAN region at all three of its wavelengths, in addition to a rich stellar population.

In order to obtain a complete YSO membership list, including confirmation of Spitzer-selected objects and identification of those members that have lost their disks, one needs additional observations, such as optical photometry. As it pertains to the present discussion, IRAC and optical data from G09 are included below. A forthcoming paper will include optical and/or nearIR spectroscopy of many of these candidates to confirm or refute their YSO status. Several investigators (e.g., the Palomar Transient Factory, PTF, see, e.g., Covey et al. 2011 or Miller et al. 2011) are monitoring this region for variability, which can also be used to confirm (or refute) a given object as a YSO.

We first present the observational details for the MIPS observations of the NAN complex (Section 2), with special attention to the extended emission (Section 2.3) and inclusion of ancillary data (Section 2.5). Then we discuss the overall properties of the point-source catalog, including issues of contamination (Section 3). We select YSO candidates in Section 4, primarily using MIPS, but including ancillary data where possible. We describe the ensemble of YSO candidates in Section 5 and define three new clusters in Section 6. We have a few brief words on the source(s) potentially powering the nebula in Section 7. Finally, we summarize our main points in Section 8. We compare our results to those obtained from other mid-IR large survey missions (for point sources and extended emission) in the Appendices.

\section{OBSERVATIONS, DATA REDUCTION, AND ANCILLARY DATA}

\subsection{Observations}

The MIPS observations of the NAN complex were conducted in two epochs. The first (and largest) component took place on 2006 June $10-11$ and covered $\sim 6 \mathrm{deg}^{2}$ in the region of highest extinction (see additional discussion in G09). These observations were part of Spitzer program id 20015 (PI: L. Rebull); the seven AORKEYs ${ }^{7}$ appear in Table 1. Additional observations were obtained on 2008 June 24 as part of program 462 (PI: L. Rebull) in an effort to increase the legacy value of this data set. They were designed to cover the corner of the map to the same integration time as the main map and added about a square degree to the total coverage. These two additional AORKEYs also appear in Table 1 and are referred to as the "patch data."

The center of the final $\sim 7 \mathrm{deg}^{2}$ map is (J2000) right ascension (R.A.; $\alpha$ ), declination (decl.; $\delta$ ) = 20:54:18, +44:05:00 $(313.575,44.0833)$, or galactic coordinates $l, b=84.8,-0.53$, or ecliptic coordinates (J2000) $337.9,+57.7$. Since this region is at a high ecliptic latitude, the field of view rotates by about a degree per day. In order to make AOR coverage as independent as possible without constraints, map offsets (see Table 1) were used along with a group-within constraint to ensure even coverage and minimal overlap between tiles. Also as a result of the high ecliptic latitude, asteroids are not a significant concern; we have just one epoch of observations per field. Figure 1 shows the region of three-band coverage with MIPS, and the four-band coverage with IRAC (discussed in G09). There are about $6 \mathrm{deg}^{2}$ with both MIPS and IRAC coverages.

Medium-rate MIPS scan maps were obtained with the spacing between adjacent scan legs set at $160^{\prime \prime}, 50 \%$ of the $24 \mu \mathrm{m}$ detector width. The total integration times were $84 \mathrm{~s}, 42 \mathrm{~s}$, and $\sim 4 \mathrm{~s}$ at each point in the map, respectively, for 24,70 , and $160 \mu \mathrm{m}$. These observations therefore can detect photospheres at $24 \mu \mathrm{m}$ down to about $3 M_{\odot}$ at the distance of the NAN complex. Much lower-mass protostars with infrared excesses due to a circumstellar disk can also be detected; typically, we can detect down to $\sim 0.3 M_{\odot}$ (mid- $M$ ) stars with IR excesses. IRAS data, though incomplete in this region (see Appendix A), show $100 \mu \mathrm{m}$ emission peaks up to $1800 \mathrm{MJy} \mathrm{sr}^{-1}$, so we did

\footnotetext{
7 AORKEYs are the unique eight-digit identifier for the Astronomical Observation Request (AOR), which can be used to retrieve these data from the Spitzer Archive.
} 


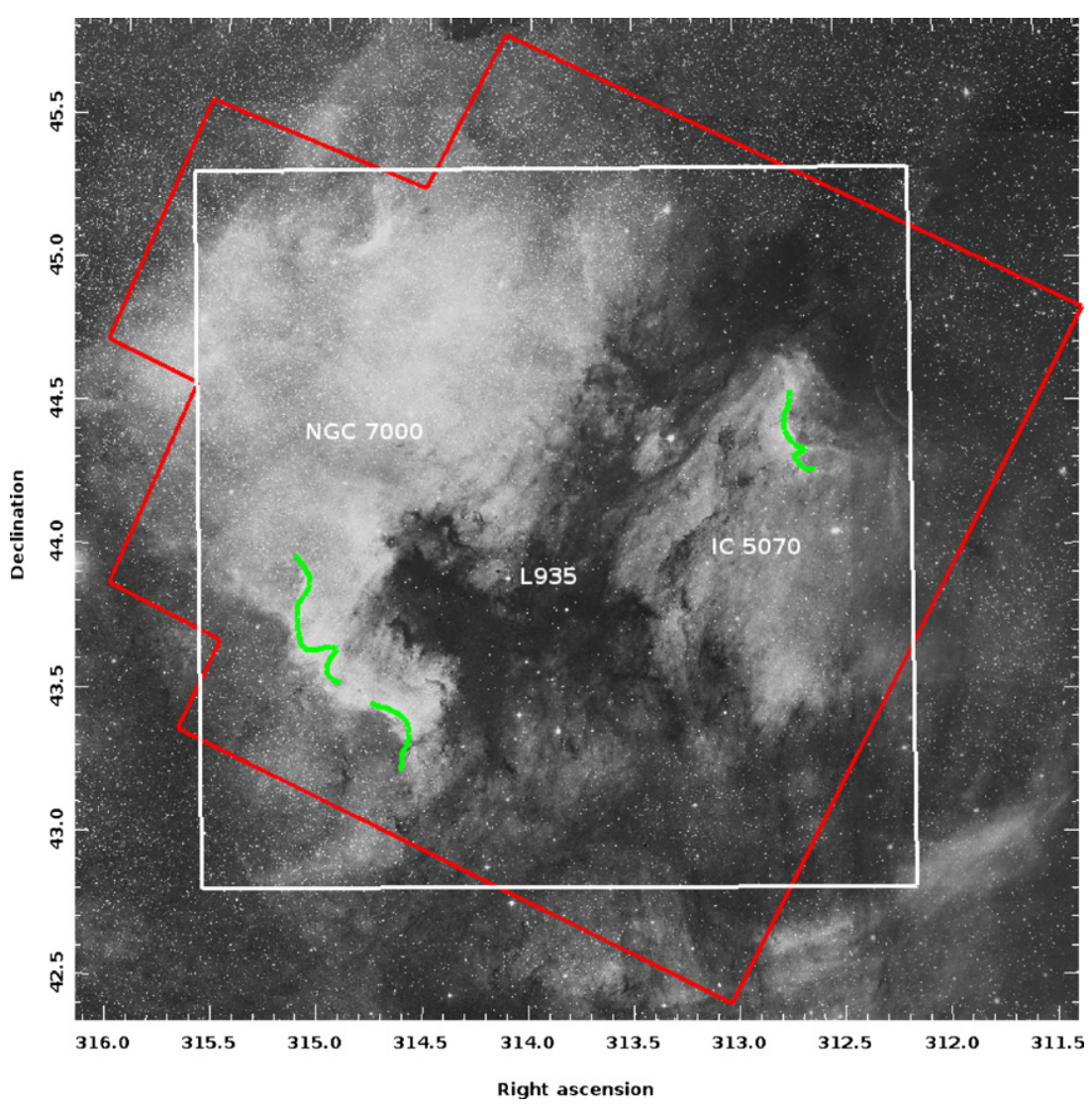

Figure 1. Approximate location of MIPS (red, irregular polygon) and IRAC (white rectangle) coverage, superimposed on a Palomar Observatory Sky Survey (POSS) red image. Three regions are indicated: NGC 7000, IC 5070, and L935, as well as some of the brightest edges (upper right and lower left, irregular contours) seen in the infrared images below (Figures 2-5) to guide the eye. The coordinates are J2000 decimal degrees.

not expect to retrieve valid (unsaturated) $160 \mu \mathrm{m}$ data, and thus did not plan on complete coverage in that band. However, these data (both the original and the secondary "patch" data) were taken in cold MIPS campaigns and, although undersampled and saturated in places, the smoothed $160 \mu \mathrm{m}$ data are still valid representations of the emission, see below.

The IRAC observations and data reduction are discussed in G09. We note that since the publication of that paper, we identified a minor error in our analysis where some objects were erroneously identified as saturated at $8 \mu \mathrm{m}$, and the total size of the map was overestimated. In the discussion below, when the G09 selection methodology is included, it is meant to be the G09 selection methodology as imposed on the corrected catalog.

We note for completeness that the four channels of IRAC are 3.6, 4.5, 5.8, and $8 \mu \mathrm{m}$, and that the three channels of MIPS are 24,70 , and $160 \mu \mathrm{m}$. These bands can be referred to equivalently by their channel number or wavelength; the bracket notation, e.g., [24], denotes the measurement in magnitudes rather than flux density units. Further discussion of the bandpasses can be found in, e.g., the Instrument Handbooks, available from the Spitzer Science Center (SSC) Web site.

\subsection{Data Reduction}

Figures 2-4 show the individual mosaics by channel, and Figure 5 shows a three-color image with all three MIPS channels included. To guide the eye, the labels for IC 5070, L935, and NGC 7000 appear in the same location in Figures 1 and 2 (optical and $24 \mu \mathrm{m}$ ); the bright emission contours on the upper

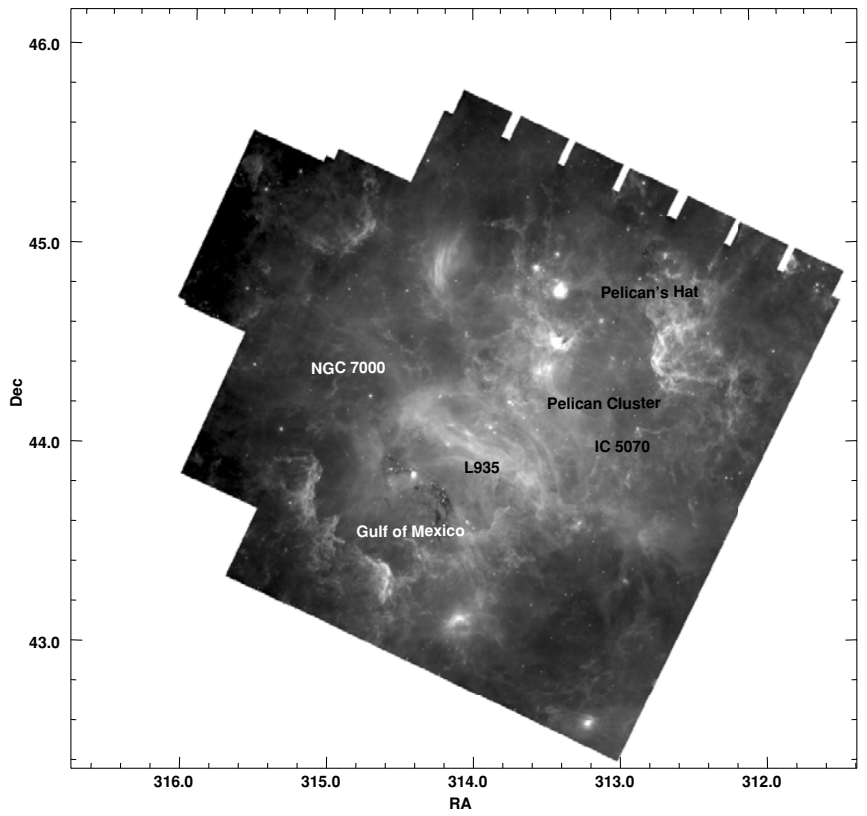

Figure 2. Mosaic of the NAN at $24 \mu \mathrm{m}$. The gray-scale colors correspond to a histogram-equalized stretch of surface brightnesses. The labels identifying IC 5070, L935, and NGC 7000 are in the same locations as Figure 1. Three of the clusters which will be discussed below are indicated as well: the Gulf of Mexico, the Pelican's Hat, and the Pelican Cluster.

left and lower right seen in all three MIPS bands are indicated in Figure 1. There is substantial extended emission in all three MIPS channels throughout the MIPS maps; see Section 2.3. 


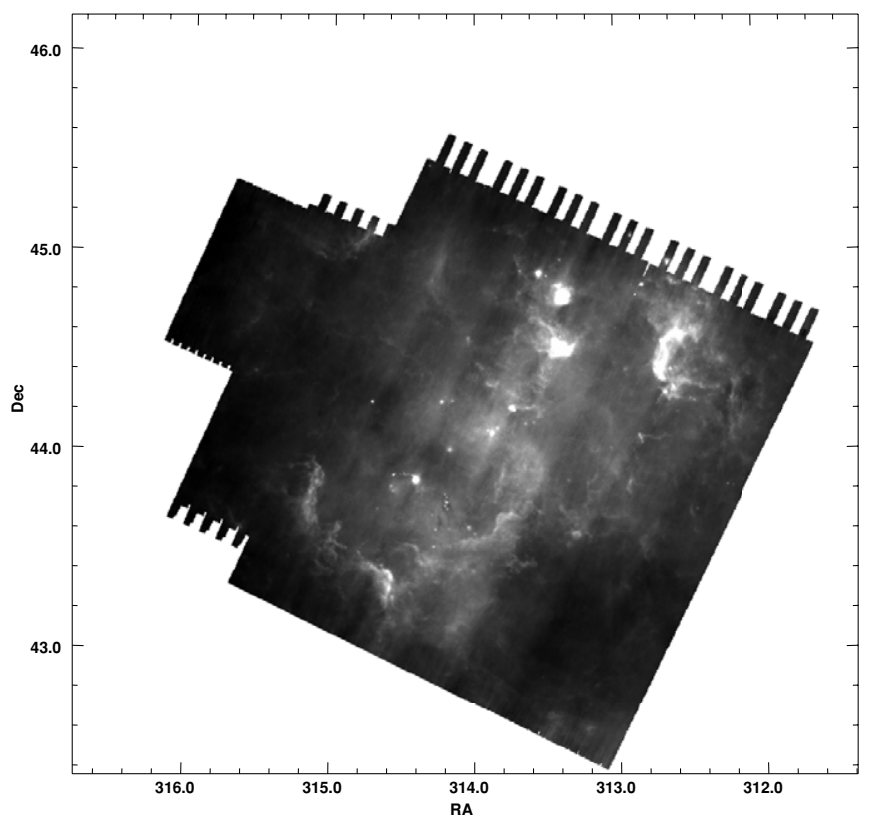

Figure 3. Mosaic of the NAN at $70 \mu \mathrm{m}$. The gray-scale colors correspond to a histogram-equalized stretch of surface brightnesses.

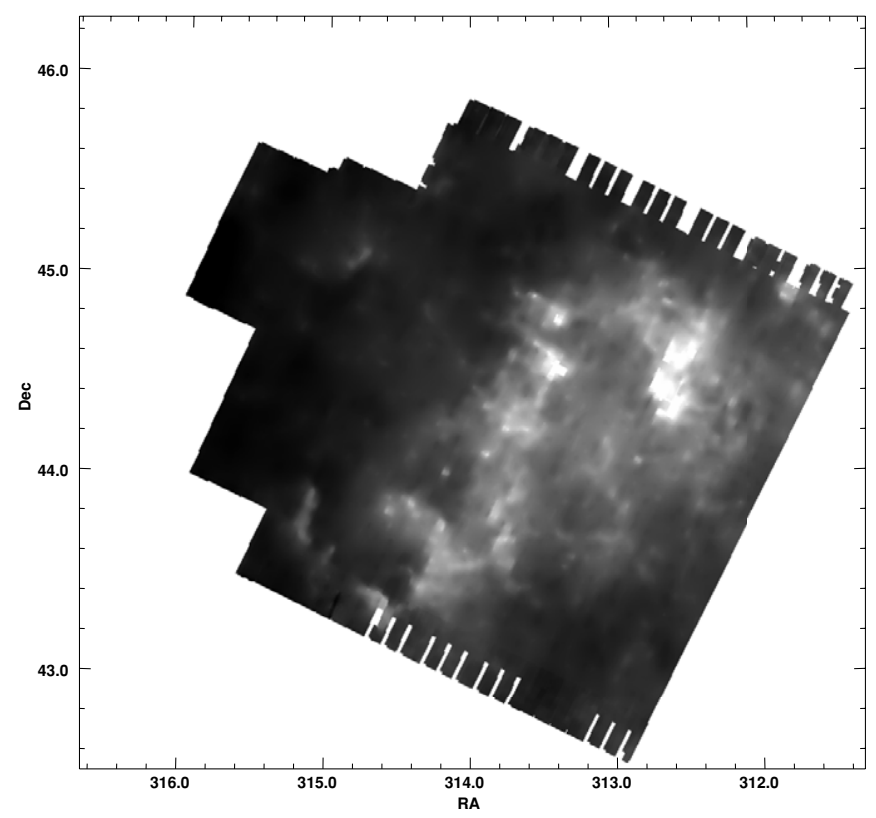

Figure 4. Mosaic of the NAN at $160 \mu \mathrm{m}$. The gray-scale colors correspond to a histogram-equalized stretch of surface brightnesses. Note that this image is highly smoothed; see the text.

\subsubsection{4 um Mosaics and Point-source Photometry}

We started with the SSC-pipeline-produced basic calibrated data (BCDs); for the main portion of the map, the data were processed with pipeline version S16.1, and the additional BCDs from the "patch" AORs were processed with pipeline version S18.0.2. Functionally, there is no significant difference between these pipeline versions. For a description of the MIPS pipeline, see Gordon et al. (2005). We constructed a mosaic from the BCDs and then performed point-source extraction on the mosaics using the SSC mosaicking and point-source extraction (MOPEX) software (Makovoz \& Marleau 2005), specifically the APEX 1-Frame routines.

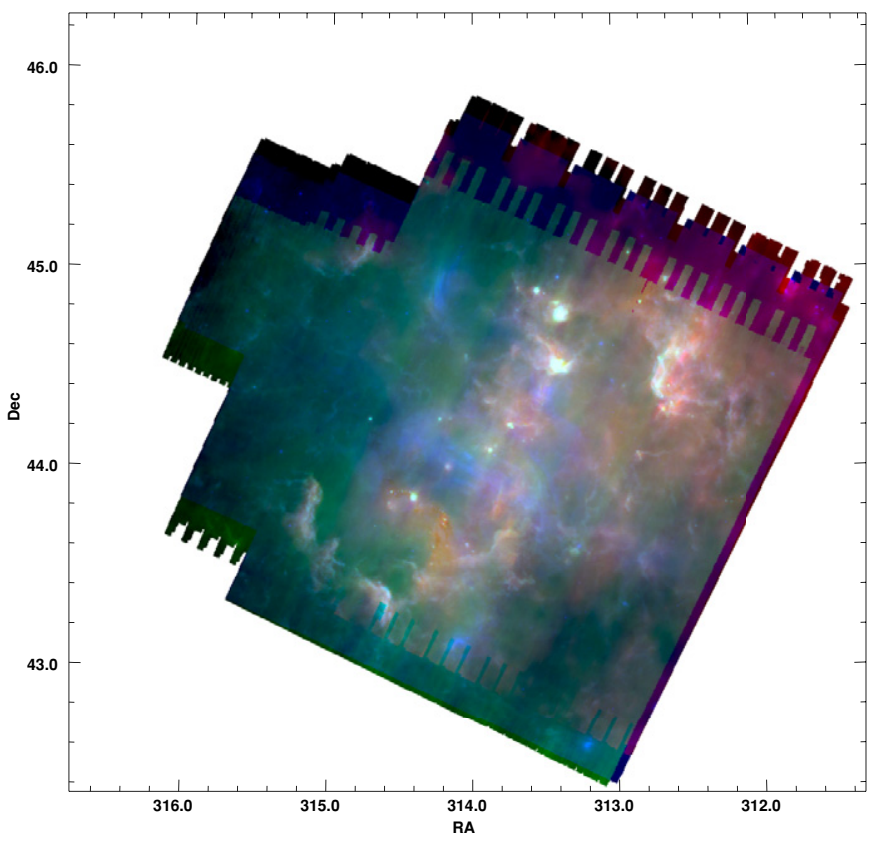

Figure 5. Three-color mosaic of the NAN including all three MIPS bands.

Constructing the mosaics at $24 \mu \mathrm{m}$ was straightforward. We used MOPEX's point-response function (PRF) fitting to obtain photometry for all of the point sources in the map in a twostep process in order to optimize parameters in regions of high nebulosity and/or source density, e.g., the Gulf of Mexico. Since the structure in the nebulosity is very complex, often of width comparable to the PRF, it can fool the point-source detection algorithm into finding false "chains" of sources along the nebulosity. As for the MIPSGAL project (Carey et al. 2009), we used a very small median filter box over which to extract the sources (APEX parameter "extract_medfilter" set to 11 pixels, where the pixels are 2 .'5, very close to the native pixel scale) to limit the influence of the nebulosity. While this does indeed limit the noise from the nebulosity, it requires a $3 \%$ correction to the derived flux densities to match flux densities obtained with a PRF fitting using a larger median filter box. We visually inspected each of the sources, dropping those along nebulous filaments that were clearly not true point sources. Imposing a signal-to-noise ratio $(\mathrm{S} / \mathrm{N})$ cutoff $\sim 10$ removes most such sources while leaving the true sources, but additional hand inspection is required to remove false sources and add real sources with a calculated $\mathrm{S} / \mathrm{N}$ comparable to but below 10 . In very crowded regions such as the Gulf of Mexico, a larger median filter box (and therefore no flux density correction) is required. We reprocessed the mosaic using these different parameters, visually inspected the detected sources, and merged the source list from this reduction with that from above.

We estimate internal (statistical) $24 \mu \mathrm{m}$ flux density errors from the MOPEX-reported $\mathrm{S} / \mathrm{N}$ divided into the measured flux density. The systematic uncertainty on the absolute flux densities at $24 \mu \mathrm{m}$ is estimated to be $4 \%$ (Engelbracht et al. 2007); we added this error in quadrature.

There were $\sim 4270$ total real point sources automatically detected at $24 \mu \mathrm{m}$, ranging from $1.4 \mathrm{mJy}$ to $4 \mathrm{Jy}$ (beyond the generally quoted saturation limit; see below). In addition to those sources automatically detected by MOPEX using the multi-step process described above, we manually added several sources to the list. For the brightest end (brighter than $\sim 1 \mathrm{Jy}$ ), MOPEX 
has a tendency to detect multiple sources when in reality there is only one very bright source. For those, we did aperture photometry and allowed recentering on the true peak of the object, taking those flux densities over the PRF-fitted flux densities. At the faint end, in the regions of the Gulf of Mexico and the Pelican cluster (see below for cluster definitions), there were several sources that were obvious by eye, but were not automatically extracted by MOPEX due to local brightness variations or source density. Flux density measurements for those objects were also manually obtained and added to the source list.

There are 4334 sources in the complete $24 \mu \mathrm{m}$ point-source catalog. The $24 \mu \mathrm{m}$ source surface density is about 600 sources $\mathrm{deg}^{-2}$. The zero point ${ }^{8}$ used to convert these flux densities to magnitudes was $7.14 \mathrm{Jy}$, based on the extrapolation from the Vega spectrum as published in the MIPS Data Handbook. We note for completeness that there are also several hundred detections that are apparently resolved and/or confused with bright localized nebulosity (knots), and thus do not appear in our point-source catalog. A detailed analysis of these objects and an assessment of whether or not they are true point sources embedded in nebulous material (and, if so, whether they are associated with the NAN complex) is beyond the scope of this paper.

The faint limit of the catalog of $24 \mu \mathrm{m}$ sources is a function of the nebular brightness, which varies across the field. Similarly, the saturation limit for point sources with MIPS-24 is also a function of location in the cloud because the total flux density registered by the detector is that due to the point source itself plus any surrounding extended nebular emission. For objects that are very bright, the MIPS pipeline attempts to reconstruct the brightest portions of the mosaic from the initial $1.0 \mathrm{~s}$ of exposure time, and therefore very bright sources can have reasonably accurate flux densities (although less accurate than fainter objects). The extended emission at $24 \mu \mathrm{m}$ varies from $\sim 74 \mathrm{MJy} \mathrm{sr}^{-1}$ in the brightest part of the Pelican Nebula to 52 $\mathrm{MJy} \mathrm{sr}^{-1}$ in the "streaks" in the center of the image (see Figure 2) to $\lesssim 26 \mathrm{MJy} \mathrm{sr}^{-1}$ in the darker parts of the cloud. An additional issue when considering completeness is the resolution; the resolution of MIPS-24 ( $\sim 7^{\prime \prime}, 2^{\prime \prime}$.5 pixel size) is poorer than IRAC or 2MASS $\left(\sim 2^{\prime \prime}\right)$. Source multiplicity and confusion will also affect the completeness of the catalog, particularly in dense regions.

\subsection{2. $70 \mu \mathrm{m}$ Mosaics and Point-source Photometry}

We started with the SSC-pipeline-produced BCDs processed with pipeline version S14.4 or later; as for $24 \mu \mathrm{m}$, functionally, there is no significant difference among these pipeline versions.

For the germanium channels (both 70 and $160 \mu \mathrm{m}$ ), the data must be treated carefully due to the time-dependent response of the arrays, the stimulator latencies, and bright source stimulator artifacts. The steps to improve the Ge:Ga data are described in the MIPS Data Handbook, available at the SSC Web site.

Some additional steps have been applied to the $70 \mu \mathrm{m}$ data that have been used and tested in the processing of the MIPSGAL Legacy data (R. Paladini et al. 2011, in preparation). These steps include (1) a "delta flat" between stimulator flashes to correct time-dependent gain variations, (2) a stim outlier

\footnotetext{
8 Zero points are used in the following standard formula to convert between flux densities and magnitudes: $m=2.5 \times \log \frac{F_{\text {zeropoint }}}{F}$ where $m$ is magnitude and $F$ is flux density.
}

rejection to correct corrupted stims during the calibration process, and (3) de-striping using a Fourier algorithm using "ridgelets" to remove the stripes left by "rowdy pixels" during the mapping procedure. (For more information on ridgelets or rowdy pixels, as well as the reduction procedure, see R. Paladini et al. 2011, in preparation.) The pixel scale of this mosaic is the native pixel scale of 9". pixel $^{-1}$. This cleaned mosaic is shown in Figure 3.

We also used MOPEX to construct mosaics from the filtered and unfiltered BCDs generated by the SSC pipeline. We resampled these pixels to be $4^{\prime \prime}$ square, which can better enable source extraction. We used a PRF defined from clean and bright point sources selected from other identically produced mosaics of similar regions, and performed point-source detection and extraction using MOPEX (specifically APEX 1-Frame) on the mosaic. The initially produced source list was cleaned for instrumental artifacts via manual inspection of the $70 \mu \mathrm{m}$ image and comparison to the $24 \mu \mathrm{m}$ image; e.g., if there was some question as to whether a faint object seen at $70 \mu \mathrm{m}$ was real or an instrumental artifact, and comparison to the $24 \mu \mathrm{m}$ image revealed a $24 \mu \mathrm{m}$ source, then the $70 \mu \mathrm{m}$ object was retained as a real source. This catalog has the same limitations as was found at $24 \mu \mathrm{m}$; the brightness of the nebulosity can drown out the faintest objects and contribute to saturation of the brightest point-source objects. The resolution at $70 \mu \mathrm{m}\left(\sim 20^{\prime \prime}\right)$ is coarser than at $24 \mu \mathrm{m}\left(\sim 7^{\prime \prime}\right)$, which complicates source matching to shorter bands and source extraction in confused regions. For all of these reasons, the $70 \mu \mathrm{m}$ catalog is neither complete nor unbiased, particularly in the regions of bright interstellar medium (ISM) or the faintest end. The estimated uncertainty on the $70 \mu \mathrm{m}$ point-source flux densities is $20 \%$. No color corrections were applied.

There were 97 total point sources detected at $70 \mu \mathrm{m}$, ranging from $\sim 0.15 \mathrm{Jy}$ to $\sim 90 \mathrm{Jy}$ (beyond saturation); most $(95 \%)$ of the sources detected are $\sim 9$ Jy or less, far below saturation. The surface density is about 15 sources $\mathrm{deg}^{-2}$. All of the $70 \mu \mathrm{m}$ objects had identifiable counterparts at $24 \mu \mathrm{m}$. (Four additional objects were flagged as potential point sources in the $70 \mu \mathrm{m}$ map but were resolved into highly nebulous bright clumps at $24 \mu \mathrm{m}$, and thus were dropped from the final catalog.) The zero point used to convert the flux densities to magnitudes was $0.775 \mathrm{Jy}$, based on the extrapolation from the Vega spectrum as published in the MIPS Data Handbook.

\subsection{3. $160 \mu \mathrm{m}$ Mosaics}

For the $160 \mu \mathrm{m}$ data, we used the same MIPSGAL techniques described above (from R. Paladini et al. 2011, in preparation) to create a clean mosaic. An initial mosaic was created with $16^{\prime \prime}$ pixels from the S18.12 data, then it was de-striped and wavelet-de-noised. As described above, the $160 \mu \mathrm{m}$ map is actually unfilled; $28 \%$ of the map is missing ("NaN" pixels) due to incomplete coverage, the missing readout in the array (see MIPS Instrument Handbook), or saturation. In order to "fill in" these gaps in the map, we used a two-dimensional $7 \times 7$ (native) pixel boxcar median to interpolate across missing ("NaN") pixels. Although this methodology substantially redistributes the flux density such that point-source photometry is no longer possible, morphological information is preserved. Some regions appear as if they might have point sources, but it is impossible to tell whether or not they are resolved given that the image is smoothed. Note that several regions are saturated. 


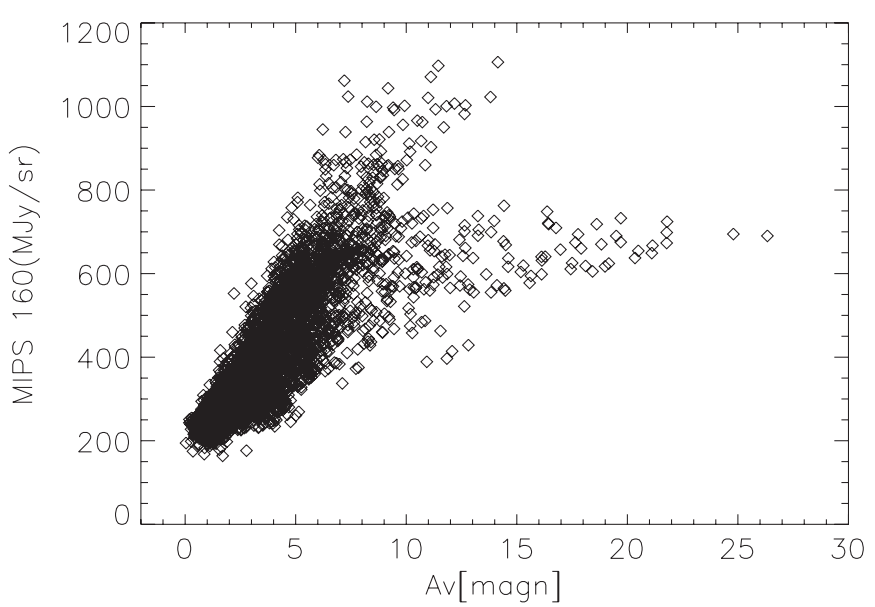

Figure 6. Plot of $A_{V}$ (from Cambrésy et al. 2002) against $160 \mu \mathrm{m}$ surface brightness, in bins of 20 pixels. As in other star-forming regions, the one-to-one correlation holds to $A_{V} \sim 5 \mathrm{mag}$. The $160 \mu \mathrm{m}$ emission closely follows the $A_{V}$ in space as well.

\subsection{Large-scale Extended Emission and Texture in the Maps}

\subsubsection{Morphology}

Bright, complex, extended emission is present at all three wavelengths throughout the MIPS NAN mosaics (Figures 2-5).

At $24 \mu \mathrm{m}$, the diversity of textures found in the ISM is stunning. There are dark lanes in the Gulf of Mexico and north of the brightest part of the Pelican (see Section 6); there are broad arcs near 314.25, $44.93(20: 57: 00+44: 55: 48)$ that somewhat resemble the ISM in the Pleiades; there are striations near the center of the map that seem to indicate large arcs centered on the Gulf of Mexico; there are highly localized nebulous very bright blobs; and, finally, there is overall turbulent ISM structure that appears to be illuminated by a source near the center of the map. The brightest portions so illuminated are to the northwest (forming the neck of the Pelican), and the southeast (forming the west coast of Mexico, the "Mexican Riviera"); these edges are indicated in Figure 1 for comparison.

Much of the brightest structure seen at $24 \mu \mathrm{m}$ is also seen at $70 \mu \mathrm{m}$. Several of the highly localized nebulous very bright blobs are still resolved at $70 \mu \mathrm{m}$. The brightest portions of the ISM at $70 \mu \mathrm{m}$ are not only the Pelican's neck and the Mexican Riviera, but also the region near several "Carribbean islands." The $24 \mu \mathrm{m}$ striations seen in the middle of the map and to the northeast are not apparent in the $70 \mu \mathrm{m}$ map.

At $160 \mu \mathrm{m}$, the brightest parts of this map are the Pelican's neck and two of the highly localized nebulous very bright blobs. The Mexican Riviera and the Carribbean islands are still discernible, even in the highly smoothed map. As in other star-forming regions, the $160 \mu \mathrm{m}$ emission closely follows the $A_{V}$ extinction contours, as derived by Cambrésy et al. (2002). Plotting $A_{V}$ values against $160 \mu \mathrm{m}$ surface brightness, Figure 6, yields a result similar to other star-forming regions. As in molecular clouds such as Taurus (Flagey et al. 2009), the oneto-one correlation holds to $A_{V} \sim 5 \mathrm{mag}$, perhaps higher. For large $A_{V}$, we see the "saturation effect" of the near-IR measurements, i.e., the near-IR does not accurately portray the dusty/cold ISM beyond $A_{V} \sim 10$.

In all three MIPS maps, the darkest regions are to the southwest and far east, where little ISM and few point sources at $24 \mu \mathrm{m}$ (none at the other MIPS bands) are apparent. To the southwest, since the star counts at IRAC bands are not dramatically higher in this region, we suspect that we are not seeing the edge of the cloud, but perhaps a part of the cloud that has not yet begun star formation in earnest. The farthest east, darkest region seen in MIPS does not have IRAC coverage, though we suspect that the map extends off the edge of the cloud here.

\subsubsection{Comparison to NGC 7023}

We can compare the colors of the entire NAN region with those of the classical reflection nebula/photo-dissociation region (PDR) NGC 7023 (e.g., Werner et al. 2004a).

We assume $\sim 5 \%$ uncertainties in the measurements themselves, dominated by the absolute calibration. The overall $8: 24 \mu \mathrm{m}$ ratio is quite similar for the NAN (0.414) and NGC 7023 (0.444), as is the $24: 70 \mu \mathrm{m}$ ratio (NAN, 0.280; NGC 7023, 0.234 ). We interpret this as that the relative number of polycyclic aromatic hydrocarbons (PAHs) to "Very Small Grains" (VSG; $\sim 1.2-15 \mathrm{~nm})^{9}$ is similar in the two regions and that the NAN's properties are similar to that of a PDR (consistent with the image morphology).

The 70:160 $\mu \mathrm{m}$ ratio, however, is much different -0.320 for the NAN and 0.546 for NGC 7023. Even if the uncertainties are $\sim 20 \%$, these values are still significantly different. This suggests that colder dust (inferred from the stronger $160 \mu \mathrm{m}$ continuum) arising from "Big Grains" (BG) in the NAN brings down the ratio. This seems to be consistent with the "voids" of emission in the images of the region, which could indicate even colder dust, particularly on the southwest corner of the image. This kind of behavior can be seen in Herschel images (e.g., Paradis et al. 2010).

Figure 7 compares the IRAC $8 \mu \mathrm{m}$ data with the MIPS 24 and $70 \mu \mathrm{m}$ data. In this composite, similar structures are seen at 8 and $24 \mu \mathrm{m}$, as in a PDR. One of the most remarkable things of this composite image is the "veil" or wispy structure at $24 \mu \mathrm{m}$ in the middle of the image without a counterpart from the dust tracers. Perhaps this is due to fine structure emission lines (e.g., [Fe II] at $26 \mu \mathrm{m})$. This idea is reinforced by the small bowshock at R.A. $\sim 314.25$ and decl. $\sim 43.1(20: 57,+43: 06)$.

\subsubsection{FCRAO Data}

Co-authors J.M.C. and L.A.H. obtained Five College Radio Astronomical Observatory (FCRAO) data of this region in 1998. A complete discussion of these data is beyond the scope of the present paper; Figure 8 shows that the $160 \mu \mathrm{m}$ emission and the ${ }^{13} \mathrm{CO}$ emission are well correlated.

\subsection{Bandmerging and Multi-wavelength Point-source Catalogs}

The IRAC map (G09) covers an area comparable to that covered by MIPS (see Figure 1). G09 discusses the IRAC source extractions and bandmerging with data from the 2MASS (at $1.25,1.65$, and $2.17 \mu \mathrm{m}$; Skrutskie et al. 2006). In summary, the IRAC and 2MASS point sources were matched by position with a matching radius of $2^{\prime \prime}$. The zero points we used for conversion between magnitudes and flux densities are $1594 \mathrm{Jy}, 1024 \mathrm{Jy}$,

\footnotetext{
9 The nomenclature describing the contribution of the different grain sizes to the mid/far-infrared emission is widely used and originates from the early models applied to explain the spectral energy distribution and extinction properties of the galactic ISM (Désert et al. 1990), using empirical dust models. Models and observations have grown in sophistication since then, but the overall picture holds, with a strong contribution from PAH broad emission features between 3 and $12 \mu \mathrm{m}$ (see, e.g., Tielens 2008), Very Small Grains (VSG; sizes $\sim 1.2$ to $15 \mathrm{~nm}$ ) at mid-IR wavelengths and Big Grains (BG), mostly silicates (sizes $\sim 15$ to $100 \mathrm{~nm}$ ), at the longer wavelengths.
} 


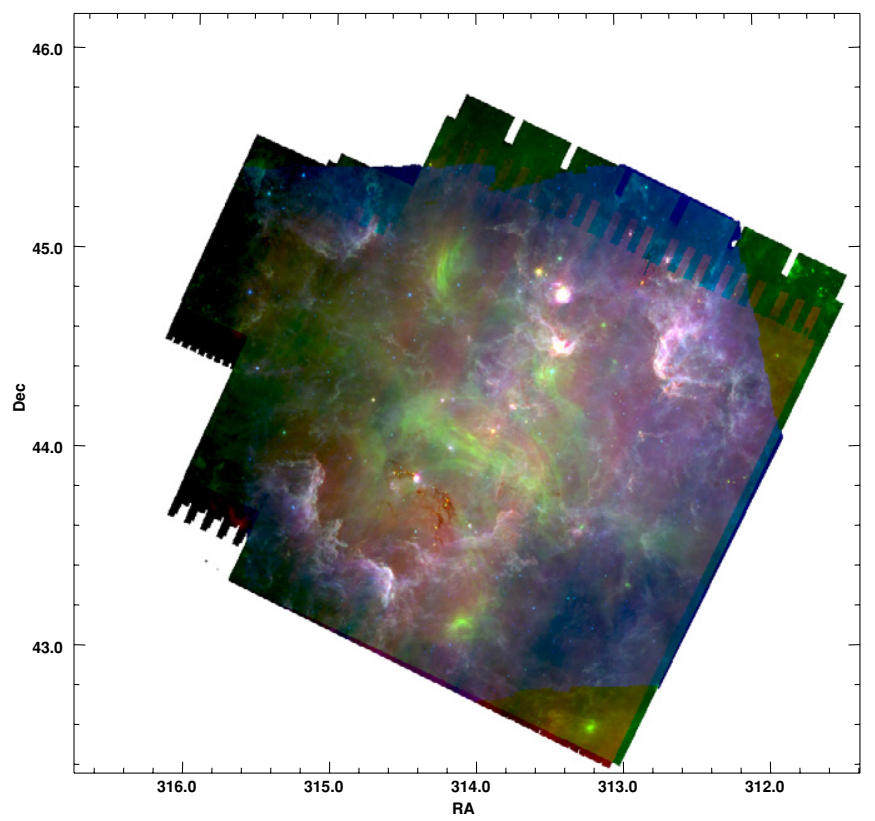

Figure 7. Mosaic of IRAC-4 (8 $\mu \mathrm{m})$, MIPS-24, and MIPS-70 data. Labels are as in earlier similar figures. Note that the 8 and $24 \mu \mathrm{m}$ emission are very similar in the large-scale PDR-like features.

and 666.7 Jy for $J H K_{s}$, respectively; for IRAC's four channels in order, they are, 280.9, 179.7, 115.0, and 64.13 Jy.

G09 also describes matching to our $B V I_{c}$ optical catalog, which is based on CCD imaging for the $\sim 2^{\circ} \times 2^{\circ}$ region in the heart of the NAN complex. There is extracted photometry for about 19,000 stars. This $B V I_{c}$ photometry is included in the spectral energy distributions (SEDs) and discussion here. The zero points we used were 4000.87, 3597.28, and $2432.84 \mathrm{Jy}$ for $B, V$, and $I$, respectively.

To match the MIPS data to the IRAC data, we have first bandmerged the $70 \mu \mathrm{m}$ detections to the $24 \mu \mathrm{m}$ detections using a $10^{\prime \prime}$ merging tolerance. By individual inspection, all the $70 \mu \mathrm{m}$ sources have $24 \mu \mathrm{m}$ counterparts. We assigned the MOPEXderived $24 \mu \mathrm{m}$ coordinates to each of the sources. Then, we merged the $24+70 \mu \mathrm{m}$ catalog to the IRAC+2MASS catalog using a tolerance of $2^{\prime \prime}$, a value determined via histograms of nearest multi-band matches and experience with other starforming regions (see, e.g., Rebull et al. 2010). (We note that the absolute pointing accuracy within a single $24 \mu \mathrm{m}$ observation is $\sim 1$ ". 4 , and the internal positional accuracy within a $24 \mu \mathrm{m}$ observation can be much smaller than that, but we have empirically found that, once multiple observations are combined and compared to other bands, $2^{\prime \prime}$ is the best value to use.)

Since G09, we have also included data from the Initial Data Release from the INT Photometric $\mathrm{H} \alpha$ Survey of the Northern Galactic Plane (IPHAS). The IPHAS survey was carried out in narrowband $\mathrm{H} \alpha$ and Sloan $r^{\prime}$ and $i^{\prime}$ filters, down to red magnitudes fainter than 20 (see Drew et al. 2005 for a general survey description). For the purposes of adding these points to our SEDs, we took the effective wavelengths for $\mathrm{H} \alpha, r^{\prime}$, and $i^{\prime}$ to be $0.656,0.624$, and $0.774 \mu \mathrm{m}$, and the zero points to be 2974.4, 3173.3, and 2515.7 Jy, respectively.

There are IRAS data over parts of out map (though it is in one of the missing "wedges"), and both MSX and AKARI data over the entire region. We considered including these data for analysis of YSO candidates, but the source density is high, and these prior missions have low enough resolution and are shallow,

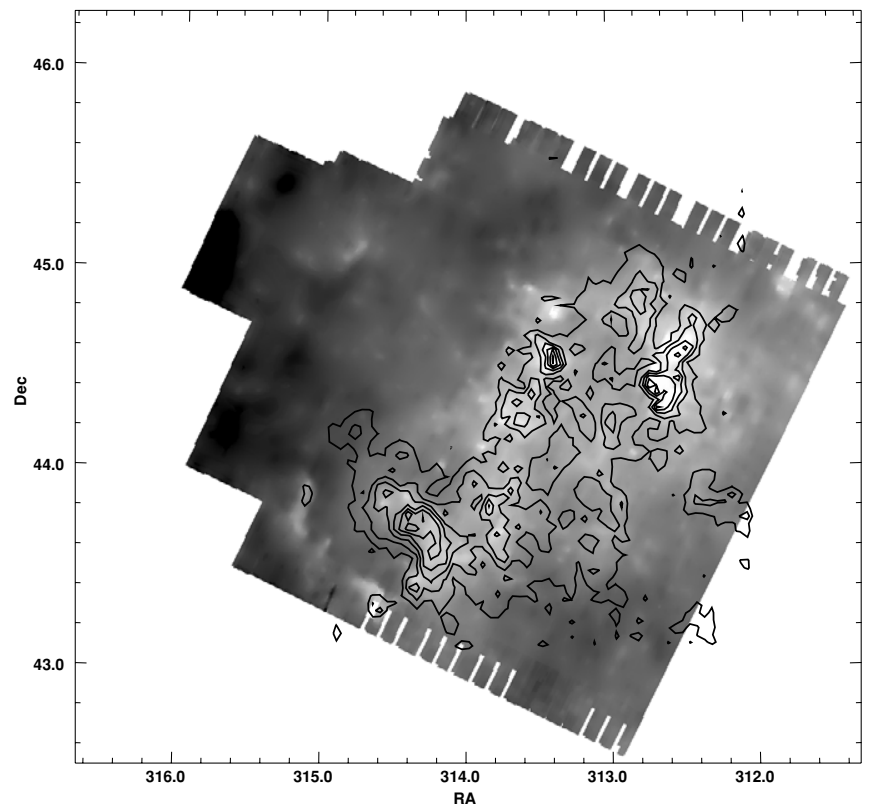

Figure 8. MIPS-160 image (gray scale) and the FCRAO ${ }^{13} \mathrm{CO}$ radio data (contours, levels 2-16). The $160 \mu \mathrm{m}$ emission is closely correlated with the ${ }^{13} \mathrm{CO}$ emission.

so we chose not to include these data in, e.g., SEDs. However, we did compare our results to those from these missions; see Appendix A.

We also included literature data from smaller (e.g., not all-sky or Galactic Plane) surveys; see the next section.

\subsection{Literature Ancillary Data}

There are several surveys of the NAN in the literature, and we compare our catalog to the literature to (1) include additional optical and near-IR data where possible, (2) identify known YSOs, and (3) identify known non-YSO (contaminant) objects. We then used the population of known YSOs and non-YSOs to inform our color-based selection of new YSOs. We now enumerate the literature surveys we included on a case-by-case basis. Where necessary (usually the very old surveys), we used original published finding charts and 2MASS to obtain updated coordinates. Some objects cannot be updated in this fashion, as there are multiple close-by objects of comparable brightness. In a few cases, we believe that certain sources from the literature do not really exist, as detailed below. (Note that prior identifications appear in Tables 3 and 9, discussed below.)

As part of a large Mount Wilson survey for Be stars, Merrill \& Burwell $(1949,1950)$ identified four Be stars in this region based on bright $\mathrm{H} \alpha$ lines as seen on objective-prism photographs. Three of the four stars are in our maps, and all three are very bright at Spitzer bands. Just one (MWC $1032=$ HD 198931) is not saturated at all four IRAC bands.

Among the first to specifically and systematically study the NAN complex itself, Herbig (1958) surveyed $6 \mathrm{deg}^{2}$ and identified 68 stars with $\mathrm{H} \alpha$ in emission, which he took to be indicative of youth. Many of these objects subsequently have become famous objects, including well-studied Herbig AeBe (HAeBe) and FU Orionis stars. Herbig's paper contains several prescient observations: (1) that the part of the complex identified as NGC 7000 is just a segment of a much larger reflection and emission nebula, (2) the exciting star(s) is (are) difficult to identify, (3) the Gulf of Mexico region may contain the exciting 
star of the $\mathrm{H}$ II region, and contains several young and very red stars. With respect to (1), the entire complex with the nebulae is now usually regarded as part of the same molecular cloud (see, e.g., Feldt \& Wendker 1993; Feldt 1993). For (2), Herbig (1958) considers and rejects several stars as the exciting source of the nebula. He concludes, agreeing with Osterbrock (1957), that the source of excitation is likely behind the Gulf of Mexico. In regards to (3), five of the stars in the Gulf of Mexico region were bright enough for Herbig (1958) to identify, and he suggested that these objects may be "deep in, or behind, the dark cloud." Additionally, as described above, Herbig (1958) identified a cluster of young objects near IC 5070, leading him to conclude that this region was the most active region of star formation in the complex. We have used 2MASS and the finding chart in Herbig (1958) to obtain updated coordinates for these objects, and they are listed in our Tables 3 and 9 as "IC 5070 cluster $N$." Other objects identified in that paper usually appear in Tables 3 and 9 as " $\mathrm{LkH} \alpha$ " numbers, as per the convention. We note here that $\mathrm{LkH} \alpha 190$ may be extended at Spitzer bands.

The next large, systematic survey of the NAN complex is Welin (1973), who, like Herbig (1958), looked for stars bright in $\mathrm{H} \alpha$. Welin (1973) surveyed a larger region than Herbig (1958), finding 141 objects down to $V \sim 16$, just 35 of which were previously known. Spectral types for several of these objects were also reported. These objects appear in Tables 3 and 9 as "UHa" numbers when no more common identification is known.

In the ground-breaking paper by Cohen \& Kuhi (1979), among observations for many other clusters, some observations are reported for objects in the NAN complex. They identify two new infrared objects (IRS 1 and IRS 2) in the NAN complex, along with two loose groupings of stars bright in the infrared: a group near LkH $\alpha 188$ (in the northern Gulf of Mexico region), and IRS 3, 4, 5, and 6 (near LkH $\alpha$ 185). These observations were difficult, obtained with a single-pixel detector drift scanning across the region. By comparison to the Palomar Observatory Sky Survey (POSS), 2MASS, and our Spitzer images, we can recover the stars identified as $\mathrm{LkH} \alpha$ 188/G1-5 (G2 and G5 are multiples), though the stars are close enough together that extracting photometry for each object individually using our current pipeline is not always possible due to resolution concerns at $8 \mu \mathrm{m}$ and longer. Of the remaining objects identified by Cohen \& Kuhi (1979), we cannot recover IRS 1-6. While of course it is possible that YSOs can vary substantially in the IR, our observations are significantly more sensitive than these early observations, and a dimming of many orders of magnitude would be possible before we would not recover them. We conclude that these objects do not really exist. The objects that do exist appear in Table 3 (and Table 9) using the same notation as Cohen \& Kuhi (1979).

In the process of obtaining $\mathrm{CO}$ observations of an expanding molecular shell in this region, Bally \& Scoville (1980) reported on 11 bright infrared sources. Using 2MASS and our data, we obtained updated positions for these objects and included them in our database, comparing them to subsequent literature as well. We note that these objects are all very bright in IRAC, often saturated. Objects 1,7 , and 8 have been identified by Comerón \& Pasqali (2005; see below) as asymptotic giant branch (AGB) stars, and thus are not YSO candidates. Bally and Scoville's object 2 is in the neck of the Pelican, and resolves with MIPS into bright nebulosity and multiple IR sources; these are likely young objects. Object 5 is a binary (one component of which is identified as an F3 by Straižys et al. 1993). Finally, object 9 appears to be the same as Object 1.
Ogura et al. (2002) reported on 32 stars bright in $\mathrm{H} \alpha$ near bright-rimmed cloud 31, which is near the Pelican Nebula. Some of these objects $(7,8,10,15,18,19,20,22,28)$ are in regions that are very bright in the Spitzer bands, and therefore may not be recoverable at some or all Spitzer bands. Some $(9,19)$ are not recovered with Spitzer. One (25) may be linked to more than one IRAC source.

Villanova et al. (2004) studied NGC 6997, which is not at the distance of the NAN complex, but is covered by our map; see Appendix B.1 below. (Note that this cluster has been commonly confused with NGC 6996; see Laugalys et al. 2006b and Corwin 2004.) Villanova et al. published $U B V R I$ photometry for 2700 stars in this region, which we have matched to objects in our catalog as optical supporting data, in addition to whether or not the objects are members of NGC 6997. The Villanova et al. (2004) optical survey reaches fainter objects than our survey does; 1200 objects from this survey do not have counterparts in our survey.

Comerón \& Pasquali (2005), in their quest to find the exciting star of the complex, studied 19 objects, six of which they determined to be AGB stars, and five more of which they determined to be carbon stars; the remaining objects were candidates for the exciting star, e.g., young high-mass stars. Both the young high-mass stars and the objects confirmed not to be young high-mass stars are useful to include in our catalog. We note here that their Object 14 appears to resolve into at least three Spitzer sources. Comerón \& Pasqali (2005) identify Bally and Scoville's Object 10 as the exciting source for the nebula.

We included in our database the spectral types for several NAN complex objects that were reported as part of large multicluster studies; Shevchenko et al. (1993) monitored HAeBes in many clusters, and Terranegra et al. (1994) obtained Strömgren photometry of Orion-population stars. Here, and in general for this database, spectral types of objects obtained via spectroscopy were allowed to supercede spectral type estimates obtained via photometry.

The group at Vilnius Observatory in Lithuania has embarked on a multi-decade campaign to obtain multiband photometry (from which they derive estimates of reddening and spectral types) for thousands of stars in star-forming regions, including hundreds of stars in the NAN complex. The Vilnius bands of observation are $U(0.345 \mu \mathrm{m}), P(0.374 \mu \mathrm{m}), X(0.405 \mu \mathrm{m})$, $Y(0.466 \mu \mathrm{m}), Z(0.516 \mu \mathrm{m}), V(0.544 \mu \mathrm{m})$, and $S(0.656 \mu \mathrm{m})$. Photometrically derived spectral types (and CCD photometry where available) from all of these papers were included in our collection of known objects: Straižys et al. (1989, 1993, 1999, 2008), Zdanavičius \& Straižys (1990), Laugalys \& Straižys (2002), Laugalys et al. (2006a, 2006b, 2007), Straižys \& Laugalys (2008a, 2008b, 2009), and Corbally et al. (2009). Note that, in addition to photometry and spectral types, Laugalys et al. (2006a) and Corbally et al. (2009) specifically identified several YSO candidates as having strong $\mathrm{H} \alpha$ emission. The adopted zero points, kindly provided by V. Straižys (2010, private communication) are, in the order UPXYZVS, 1325, 2112, 4106, 4169, 3949, 3748, and 3141 Jy.

The IPHAS team, in Witham et al. (2008), identified bright $\mathrm{H} \alpha$ sources in the first $80 \%$ of their survey, which includes the NAN region. The IPHAS team did not attempt to identify the nature of the sources, simply that they were bright in $\mathrm{H} \alpha$. Since YSOs are expected to be bright in $\mathrm{H} \alpha$, the $\sim 80$ stars from that catalog in this region were also matched to our catalog, and the information that they are bright in $\mathrm{H} \alpha$ is folded in to our assessment of YSO candidacy below. We note that some stars 
Table 2

Statistics of MIPS Point-source Detections

\begin{tabular}{lrcc}
\hline \multicolumn{1}{c}{ Item } & Number & $\begin{array}{c}\text { Fraction of } \\
24 \mu \mathrm{m} \text { Sample }\end{array}$ & Notes \\
\hline $24 \mu \mathrm{m}$ & 4334 & 1.00 & 3694 in region covered by all four IRAC bands \\
$70 \mu \mathrm{m}$ & 97 & 0.02 & \\
$24 \mu \mathrm{m} \& 70 \mu \mathrm{m}$ & 95 & 0.02 & 3100 in region covered by all four IRAC bands (84\%) \\
$24 \mu \mathrm{m} \&$ ANY IRAC band & 3243 & 0.75 & $89 \%$ of $70 \mu \mathrm{m}$ sample \\
$70 \mu \mathrm{m} \&$ ANY IRAC band & 85 & 0.02 & 2577 in region covered by all four IRAC bands (70\%) \\
$24 \mu \mathrm{m} \&$ ALL IRAC bands & 2582 & 0.60 & $61 \%$ of $70 \mu \mathrm{m}$ sample \\
$70 \mu \mathrm{m} \&$ ALL IRAC bands & 59 & 0.01 & \\
$24 \mu \mathrm{m} \&$ ANY $2 \mathrm{MASS}$ band & 2972 & 0.69 & \\
$24 \mu \mathrm{m} \&$ ANY IPHAS band & 1824 & 0.42 & \\
$24 \mu \mathrm{m} \& V$ or $I_{c}$ or $r^{\prime}$ or $i^{\prime}$ & 1931 & 0.45 & \\
\hline
\end{tabular}

with $\mathrm{H} \alpha$ in emission could be active stars or classical Be stars with gaseous disks, not necessarily young stars.

Finally, G09 identified 1657 YSO candidates based on IRAC data. Since then, we have continued to improve our catalog quality (most notably identifying some objects incorrectly marked as saturated at $8 \mu \mathrm{m}$ ), and, using the same algorithm as described in G09, we can now identify 1750 YSO candidates based just on the IRAC NAN data. Objects identified using the G09 methodology but not rediscovered using the MIPS-based search here are listed explicitly in Appendix C.

In summary, we merged our Spitzer-based catalog (from Section 2.4) to these existing data from the literature. There is literature information for about 3600 stars scattered throughout the map, including photometry and spectral types or other classification (such as if they were oxygen- or carbon-rich AGB stars, or red clump giant stars), or even just prior identifications (such as "GSC N" or "TYC N," listed in the tables below). Thus, our final multi-wavelength catalog includes MIPS, IRAC, 2MASS, IPHAS, and other optical data, both from the literature and that which we ourselves have obtained. After including all of this ancillary data, there are spectral types for more than 1200 objects in our catalog, ranging from O5 to M8, with $\sim 400$ additional generic AGB, carbon star, and red clump giant identifications. These types are largely photometric (as opposed to spectroscopically obtained), and largely for types $\mathrm{K}$ and earlier.

Just 142 previously identified objects of any sort are missing entirely from our Spitzer-based catalog (aside from those faint ones from Villanova et al. 2004); the overwhelming majority of those are saturated at all Spitzer bands (and thus do not appear in our catalog) or resolve into multiple pieces when viewed with Spitzer (and thus are not formally matched to a single Spitzer source).

Of the previously identified YSOs in the literature (identified in the tables below), it is interesting to consider what fraction are present in our database. About 200 objects in our catalog are identified specifically as previously known YSOs, though this is likely to consist of a mixture of true members and interlopers. Just $\sim 50 \%$ of them have measured flux densities in any MIPS band, and $95 \%$ of them are measured at any IRAC band. Most of the "missing" ones are saturated or in very crowded regions in the Spitzer images, which is consistent with the previous surveys being shallower and/or lower spatial resolution. (Note that Table 5 in the discussion below includes statistics on previously known objects recovered or not using G09 and the primarily MIPS-based method presented here.)

\section{ENSEMBLE STATISTICS, SOURCE COUNTS, AND CONTAMINATION}

\subsection{Catalog Statistics}

Table 2 presents MIPS-focused statistics on the ensemble catalog spanning $0.35 \mu \mathrm{m}$ through $70 \mu \mathrm{m}$. About $75 \%$ of all the MIPS-24 sources have an IRAC counterpart. However, the IRAC and MIPS maps do not cover exactly the same area. Considering just the region of the MIPS map where there are also IRAC data from all four bands, $\sim 85 \%$ of the $24 \mu \mathrm{m}$ sources have a measured counterpart at at least one IRAC band (where the $15 \%$ without an IRAC counterpart are overwhelmingly fainter than $\sim 8$ th mag, and thus clearly on the very faint tail of the distribution; see Figure 9 below); $60 \%$ of the $24 \mu \mathrm{m}$ sources have a measured IRAC flux density at all four IRAC bands. There are near-IR $J H K_{\mathrm{s}}$ data covering this entire region from 2MASS, but 2MASS is relatively shallow; $69 \%$ of the MIPS-24 sources have a $2 \mathrm{MASS}$ counterpart. (We note that this region has also been covered by the UKIRT Infrared Deep Sky Survey (UKIDSS) to much deeper limits than 2MASS; we will include these data in a forthcoming paper.) The optical data are highly affected by reddening; $45 \%$ of all the MIPS-24 sources have an optical counterpart of any sort (from our data, IPHAS, or the Vilnius photometry).

\subsection{Source Counts and Contamination}

MIPS sources in general are a combination of cloud members, foreground/background stars in the Galaxy, and the extragalactic background. Since the NAN complex is essentially in the Galactic plane $(b \sim-0.6)$ and is viewed along a spiral arm, we expect to see mostly Galactic sources, with few extragalactic objects, and we expect an excess over the Galactic background attributable to cloud members. Figure 9 shows the observed NAN 24 and $70 \mu \mathrm{m}$ differential source counts in comparison to observed source counts from the $6.1 \mathrm{deg}^{2}$ Spitzer Wide-area Infrared Extragalactic Survey (SWIRE; Lonsdale et al. 2003) ELAIS (European Large Area ISO Survey) N1 extragalactic field $^{10}$ (the cores-to-disks (c2d; Evans et al. 2003, 2009) reduction is used here, as in Rebull et al. 2007, and the distribution is scaled for relative areas but not additionally reddened). The sources that we see in the NAN do not resemble the source count distribution for extragalactic sources. For $70 \mu \mathrm{m}$, scaled source counts from the $\sim 8 \mathrm{deg}^{2}$ Vulpecula OB association (Vul OB1; Billot et al. 2010) from MIPSGAL (Carey et al. 2009) are also shown for comparison. Like the NAN, Vul OB1 is in the

$\overline{10}$ VizieR Online Data Catalog, II/255 (Surace et al. 2004). 


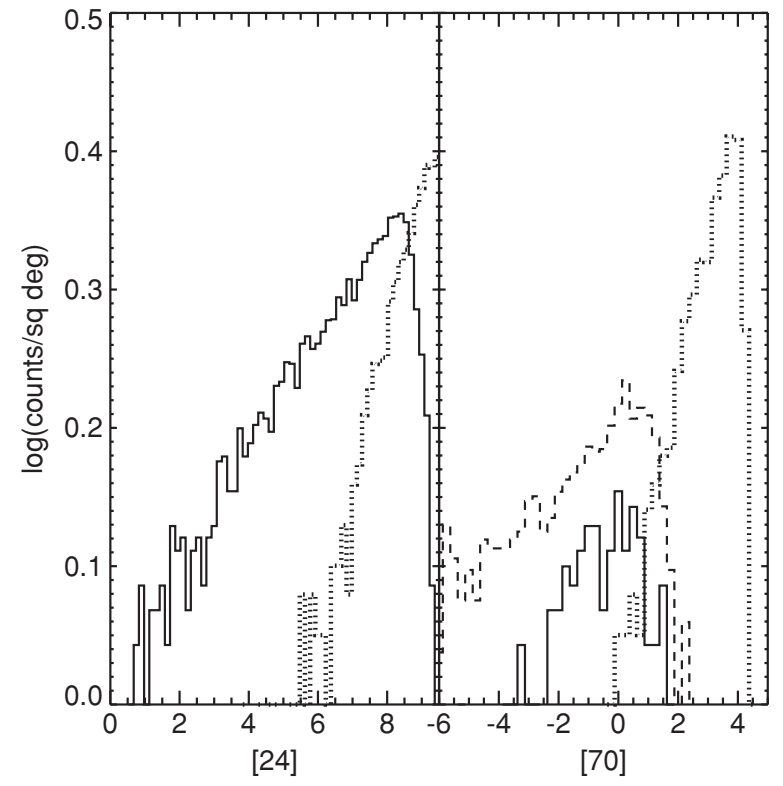

Figure 9. Differential number counts at 24 and $70 \mu \mathrm{m}$ in the NAN complex. Extragalactic background source counts from the $6.1 \mathrm{deg}^{2}$ SWIRE ELAIS N1 field are shown (scaled) for comparison (thick short-dashed line). For $70 \mu \mathrm{m}$, source counts from the $\sim 8 \mathrm{deg}^{2}$ Vul OB1 field from MIPSGAL are also shown (scaled) for comparison (long-dashed line). The sources we see in the NAN are more likely to be in our Galaxy than extragalactic sources.

Galactic plane $(l \sim 60.5, b \sim 0$ ), the sky background levels are comparable to those in the NAN, and the Spitzer observation covers an appreciable area such that there are enough sources to effect a comparison. The source counts in the NAN much more closely resemble that from a star-forming region like Vul OB1 than they do an extragalactic region like SWIRE. While the shapes of the differential source count distribution are similar between the NAN and Vul OB1, there are more sources overall, and more bright sources, in Vul OB1. In both cases, we have comparable data and data reduction strategies, and we are looking along a spiral arm. In the case of Vul OB1, the starforming region is $2.3 \mathrm{kpc}$ away, which is considerably further away than the NAN. As such, the Vul OB1 region encompasses more foreground volume, and many more foreground sources are included in the sample. (More evidence for this can be found in the fact that most of the NAN $70 \mu \mathrm{m}$ sources are in the Gulf of Mexico region, as opposed to more evenly distributed over the field.)

In order to begin to place constraints on whether we see a higher count rate of MIPS sources over the generic Galactic background (e.g., cloud members), we can compare the source counts within our own maps. The portion of the map to the northeast can be seen to be very likely to be "off-cloud" by inspection of the cloud morphology at all available bands (optical through $160 \mu \mathrm{m}$ ). The general sky brightness is lower at all bands in this region; see Figures 2-5. There are just five $70 \mu \mathrm{m}$ point sources in this very roughly $1 \mathrm{deg}^{2}$ region; extrapolating this contamination rate to the rest of the map implies that $\sim 35$ of the NAN $70 \mu \mathrm{m}$ sources may be generic Galactic background, e.g., contamination. At $24 \mu \mathrm{m}$, in the same region, there are $\sim 400$ sources, implying that as many as $\sim 2500$ of the NAN $24 \mu \mathrm{m}$ sources may be Galactic contamination, or roughly half of all of the detected point sources. However, this is likely to be a strong function both of source brightness and location in the map, and moreover sources that have infrared excesses are more likely to be cloud members than background, as discussed below.

Galactic stars contribute substantially to the MIPS source counts in NAN. This is different than many of the other nearby star-forming regions studied with Spitzer (see, e.g., the series of papers from the c2d Legacy team, such as Rebull et al. 2007 for MIPS observations in Perseus; also see Rebull et al. 2010 for Spitzer observations in Taurus) which are out of the Galactic plane. The contaminants of greatest concern to us are the ones that appear to have YSO-like colors. These include some AGB stars (oxygen- and carbon-rich); although intrinsically very bright, such that they would be saturated even at large distances, once they are reddened by the NAN cloud, they can appear fainter and have thus have colors and overall brightnesses suggestive of YSOs (see, e.g., Robitaille et al. 2008).

Some AGBs (including specifically carbon stars) are already known in the direction of the NAN; see Section 2.5 above and Figure 11 below. Although this is far from a complete sample, we can examine the number counts of these objects in our map in an effort to quantify how many AGB contaminants we might have. Twenty-five of these objects are seen in $24 \mu \mathrm{m}$, and two are seen at $70 \mu \mathrm{m}$ as well. Their colors suggest that they could indeed be confused with bright YSO candidates. All of these objects are relatively bright, e.g., have [3.6] between 6.6 and $10.7 \mathrm{mag}$, but this is likely a bias in that the previous studies tended to focus on the brighter objects.

We can also attempt to constrain the AGB contamination by comparison to other regions studied with Spitzer. Harvey et al. (2006) argue that any AGB star in our Galaxy is likely to be saturated in most IRAC observations (except for cases of very large $A_{V}$ ). Rebull et al. (2007), using data from Blum et al. (2006) obtained in the Large Magellanic Cloud as an aid to quantifying the AGB contamination in Perseus, find that a typical AGB star (in the direction of Perseus) would appear so bright as to be saturated in the 2MASS survey, but not with MIPS. Harvey et al. (2007a) argue that, in their $0.58 \mathrm{deg}^{2}$ field in Serpens (at $b \sim 5 \mathrm{deg}$ ), they expect up to six AGB stars and estimate that they actually find three. Scaling from those values to our $\sim 6 \mathrm{deg}^{2}$ field, we might expect $\sim 30-60$ AGB contaminants. However, in spectroscopic follow-up of half of the Spitzer-selected YSO candidates from Serpens, Oliveira et al. (2009) found a much higher AGB contaminant rate among the Spitzer-selected YSO candidates-25\%. Unlike Perseus, where we are looking out of the Galaxy and so there is an "edge" to the distribution of AGBs, both the NAN and Serpens have sightlines in the Galactic plane, where extinction cuts off the AGBs before the Galactic population really falls off. Extinction makes the AGBs look even more like YSO candidates, because it can make an object selected from a single color-magnitude diagram (CMD) appear to have an IR excess, and more generally because it makes the AGBs look fainter, comparable to YSOs in the cloud. Thus, a high contamination rate among the YSO candidates from AGB stars is expected, probably anywhere from the $\sim 5 \%$ estimated originally by a scaled Harvey et al. (2007a) to the $\sim 25 \%$ based on the Oliveira et al. (2009) spectroscopic follow-up of half the Spitzer-selected Serpens YSO candidates.

While of course any individual YSO candidate we find will require spectroscopic follow-up to confirm its YSO status, we conclude that even in the worst contamination case, the majority of objects selected as having red MIPS colors that we report here as YSO candidates are indeed likely to be young stars associated with the NAN cloud complex. Statistically, other 
properties (e.g., position in multiple CMDs, clustering) make it less likely that a given object is a contaminant, and more likely to be a YSO, as we discuss in the next section.

\section{SELECTION OF YSO CANDIDATES}

There is no single Spitzer color selection criterion (or set of criteria) that is $100 \%$ reliable in separating members from non-member contaminants. Many have been considered in the literature (e.g., Allen et al. 2004; Rebull et al. 2007; Harvey et al. 2007a; Gutermuth et al. 2008, 2009; Rebull et al. 2010). Some make use of just MIPS bands, some make use of just IRAC bands, most use a series of color criteria, and where possible, they make use of (sometimes substantial) ancillary data. In our case of the NAN, because it is in the Galactic plane and along a spiral arm, as discussed above, the contaminants having YSO-like colors are largely expected to be AGB stars and not galaxies; we can use that information to our advantage. We have shorter-wavelength ancillary data for many of the objects in our catalog (see Section 2.5) that can be used to constrain reddening due to extinction and separate out things resembling reddened photospheres. It is still most effective to separate the members from the diskless stars and galaxies (if not the less common AGBs) by using infrared colors as a discriminant, so we are still primarily Spitzer-driven in our source selection. Our inventory of the no or very low IR excess objects (Class III or its high-mass equivalent) is incomplete since we are using red colors (interpreted as an IR excess due to a circumstellar disk) to select members.

We imposed an IRAC-only YSO selection in G09. Now, we include MIPS data, investigating candidates found via an MIPS-based selection and comparing this to the IRAC-only YSO selection from G09. In order to construct the best possible list of YSO candidates, we start with a simple color selection, then incorporate information from the ancillary data we have amassed. A complete list of the YSO candidates selected here is included in the text below; objects selected using the IRAC-only YSO selection from G09 but not recovered here are reported separately in the Appendix.

\subsection{The [3.6] versus [3.6] - [24] Diagram}

Of the MIPS bands, $24 \mu \mathrm{m}$ data are available for the largest number of sources. In order to identify sources with excesses at $24 \mu \mathrm{m}$, we compare $24 \mu \mathrm{m}$ flux densities to those from a shorter wavelength band.

In recent papers such as Rebull et al. (2007) or Rebull et al. (2010), the $K_{s}$ versus $K_{s}-$ [24] CMD was used to find YSO candidates. In cases of nearby star-forming regions, or regions where there are no IRAC data, this can be effective. However, there are some good reasons to use [3.6] versus [3.6]-[24] rather than $K_{s}$ versus $K_{s}-$ [24]. There is an intrinsic spread in $K_{s}-$ [24] photospheric colors that is not present in [3.6]-[24] because late-type stars are not colorless at $K_{s}-$ [24] (Gautier et al. 2007). The effects of reddening are stronger at $K_{\mathrm{s}}$ than at $3.6 \mu \mathrm{m}$. And, if 2MASS is the only source of $K_{\mathrm{s}}$, even short $3.6 \mu \mathrm{m}$ integrations can reach fainter sources than $2 \mathrm{MASS}$ does. In the specific case of the NAN complex, the area covered by MIPS is well matched to that by IRAC, we have substantial reddening, and 2MASS is shallow with respect to the brightnesses expected for late-type young stars at the distance of the NAN (and with respect to the depth of our IRAC data; see Table 2). For the above reasons, we use [3.6] versus [3.6]-[24] as our primary mechanism for selecting YSO candidates.

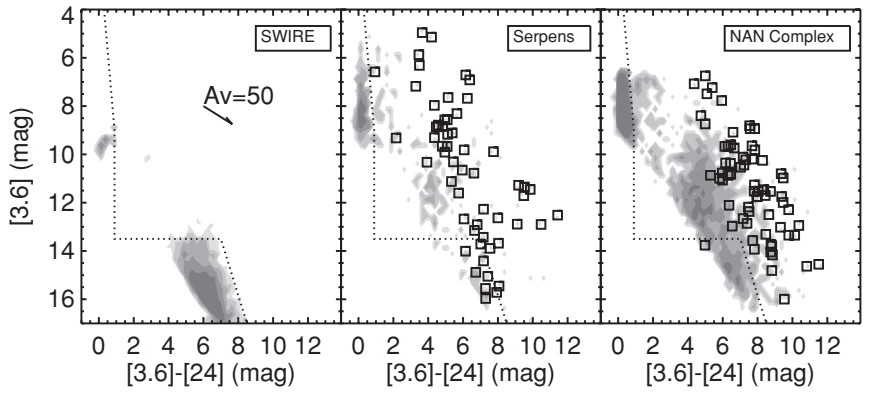

Figure 10. [3.6] vs. [3.6] - [24] contour plots for objects in SWIRE (left; $l=85^{\circ}, b=+45^{\circ}, 6.1 \mathrm{deg}^{2}$ ), Serpens (middle; $l=32^{\circ}, b=+5^{\circ}, 0.85 \mathrm{deg}^{2}$ ), and the entire NAN complex (right; $l=85^{\circ}, b=-0.6 ; \sim 7 \mathrm{deg}^{2}$ ). Objects in SWIRE are expected to be mostly galaxies (objects with [3.6] $\gtrsim 14$ ) or stellar photospheres (objects with [3.6] - [24] $\lesssim 1$ ). A box denotes that the underlying object was also detected in $70 \mu \mathrm{m}$. Objects that are the most obvious candidate young objects have colors unlike those objects found in SWIRE, e.g., [3.6] $\lesssim 14$ and [3.6] $-[24] \gtrsim 1$, or very red colors if [3.6] $\gtrsim 14$. The dotted line denotes the dividing line between the region clearly occupied by SWIRE-type contaminants (galaxies and diskless stars) and YSOs.

Figure 10 shows the [3.6] versus [3.6]-[24] plot for the NAN sample in comparison to the $6.1 \mathrm{deg}^{2}$ SWIRE (Lonsdale et al. 2003) ELAIS N-1 and $0.85 \mathrm{deg}^{2}$ c2d Serpens (Harvey et al. 2007b) samples. Ordinary stellar photospheres (likely foreground or background stars) have [3.6] - [24] $\sim 0$, and the galaxies as seen in SWIRE make up the large, elongated source concentration near [3.6]-[24] 6, [3.6] 15. Objects not in this region, e.g., the brighter and/or redder objects (those to the right of the dotted line in Figure 10), are less likely to be part of the Galactic or extragalactic backgrounds, and more likely to be YSOs with a $24 \mu \mathrm{m}$ excess. Both the Serpens and NAN clouds have substantial populations of objects meeting these criteria. The distribution of objects found in the NAN is much more similar to that in Serpens than in ELAIS N-1.

Reddening can move higher mass objects from the photospheric locus into the region where lower mass YSOs are found. The reddening is far from uniform, with regions of $A_{V}$ up to $\sim 30$ mag, according to the Cambrésy et al. (2002) extinction map. The extinction is predominantly generated by the clouds in the NAN. As seen in Figure 10, even $A_{V} \sim 50$ does not move objects far enough from the photospheric locus into the region where most of the objects are found in the "red" region of the diagram (to the right of the dotted line). However, the objects that are more moderately red, those immediately to the right of the dotted line, could appear there because of reddening, as opposed to due to an excess due to a circumstellar disk. We can attempt to remove such simply reddened photospheres by incorporating the ancillary data we have collected, see below.

Given the distance to the NAN, and given the background counts analysis above, there are likely to be many legitimate NAN cloud members overlapping the region populated by the SWIRE galaxies, but it is difficult to distinguish the populations given the data we have. For this work, we limit ourselves to the more obvious candidates, e.g., primarily the objects brighter and/or redder than the dotted line in Figure 10, with the caveat that the selection is further modified, here and below. The first modification to this selection is that we look at the significance of the excess. If the excess is seen only at $24 \mu \mathrm{m}$, and the measured flux density is less than $5 \sigma$ above the expected photosphere as extrapolated from a Rayleigh-Jeans slope tied to $3.6 \mu \mathrm{m}$, it is less likely to be a real excess source. Conversely, for the objects closest to the photosphere locus (e.g., [3.6]<10), we accept as candidates those objects where the $24 \mu \mathrm{m}$ excess is more 


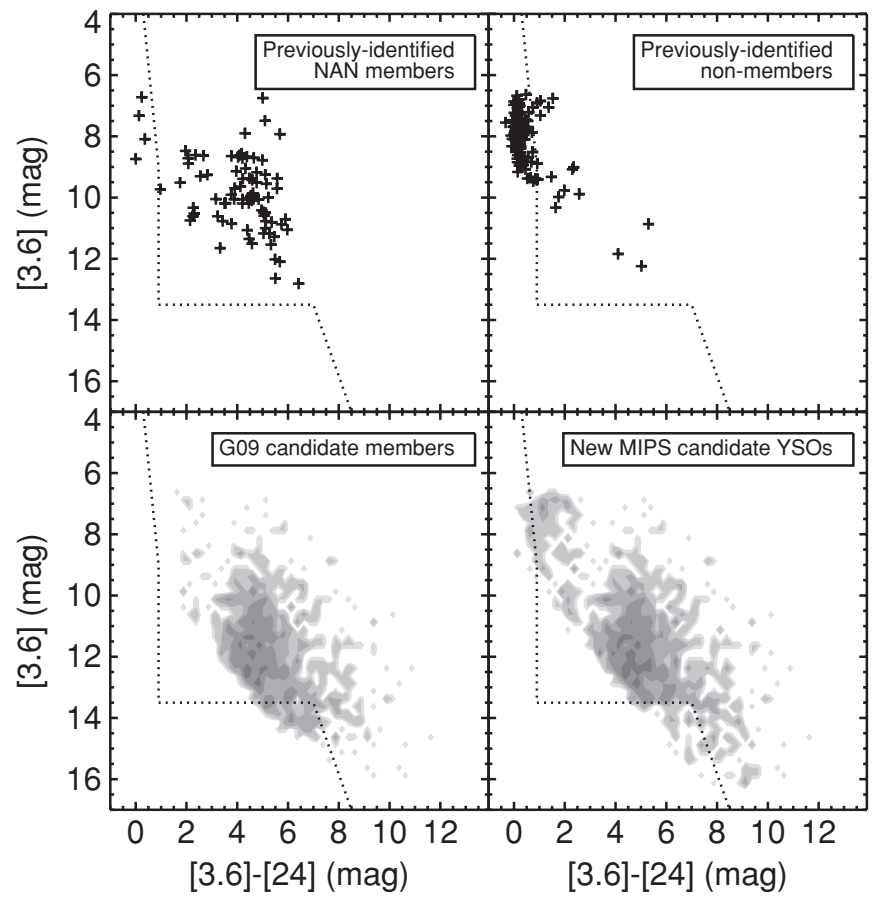

Figure 11. [3.6] vs. [3.6] - [24] plots for subsets of the NAN sample; see Figure 10 for comparison. As before, dotted line denotes the dividing line between the region clearly occupied by SWIRE-type contaminants (galaxies and diskless stars) and YSOs. Here the panels are previously identified YSOs, previously identified contaminant objects, YSO candidates from G09, and the YSO candidates identified in this work, some of which rediscover those from G09 (see Section 4.4). The distributions of YSO candidates look different than the previously identified non-members and more like the previously identified members.

than $8 \sigma-10 \sigma$ above the extrapolated photosphere, depending on whether the excess is seen at any other bands (e.g., if there is a small excess at $24 \mu \mathrm{m}$ and a clear excess at IRAC bands, this is more believably an object with a true IR excess than if there is no excess at IRAC bands, just a small excess at $24 \mu \mathrm{m}$, and no detection at $70 \mu \mathrm{m}$ ). This has the effect of omitting some sources that technically meet the color criteria and including some sources that are just blueward of the dotted line in Figure 10. There are $\sim 1200$ sources identified from 3.6 and $24 \mu \mathrm{m}$ data in this fashion.

Figure 11 compares the distribution of various subsets of the NAN data in the [3.6] versus [3.6]-[24] parameter space. Based on our collection of literature data above (Section 2.5), we have a pre-assembled set of NAN members and non-members, which are shown in Figure 11. By and large, these objects fall where we expect them to based on Figure 10-most of the NAN members appear as red in this diagram, which we interpret to mean that they have infrared excesses, and most of the non-members do not. However, it is notable that there are outliers in both cases-some non-members appear to have excesses (largely the AGB contaminants noted above), and there are some members without excesses (which were most likely detected using other mechanisms besides IR colors, such as $\mathrm{H} \alpha$, although we note that intrinsic stellar variability could also be a contributing factor). Figure 11 also shows the distribution of the YSO candidates selected in G09 as well as the distribution of YSO candidates from our final selection below (Section 4.4). Note that the candidates selected below often recover sources from G09; see Table 5 below and associated discussion. In both cases, the distribution of YSO candidate sources is similar,

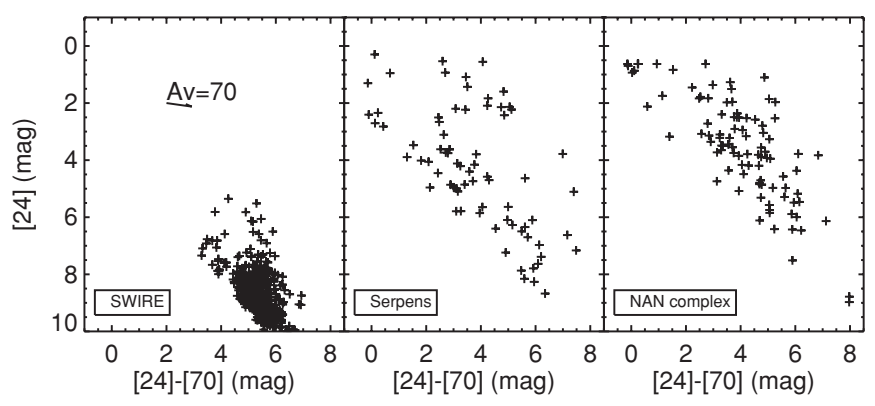

Figure 12. [24] vs. [24] - [70] plots for objects in SWIRE (left), Serpens (middle), and the entire NAN complex (right). Again, the distribution of objects in the NAN more closely resembles that from Serpens than SWIRE, e.g., are more likely to be young stars than galaxies.

with the highest density of sources found near [3.6]-[24] 5, [3.6] 12.

Figure 12 shows [24] versus [24]-[70] for the same three samples as Figure 10, namely, SWIRE ELAIS N-1, c2d Serpens, and the NAN sample. The distribution of sources found in the NAN again is a much closer match to that from Serpens than from SWIRE. Few of the objects seen at $70 \mu \mathrm{m}$ in the NAN are likely galaxies; several are very red.

\subsection{Incorporating Ancillary Data}

As discussed above (Section 2.5), we have included in our database a wide variety of ancillary data. These data are not always well matched to our Spitzer data in depth or area. But they can contribute to the assessment of any one object as to its likelihood of being a YSO.

Rebull et al. (2010), working in Taurus, had a similar problem to overcome in that there were $\sim 44 \mathrm{deg}^{2}$ of Spitzer data, considerable foreground and background contamination, but also considerable ancillary data. The approach taken in that study was to incorporate, in addition to the easily quantifiable Spitzer magnitude and color criteria, qualitative judgments based on what data were available. These included but were not limited to: relative brightness at all available bands, amplitude of excess, shape of SED, apparent (projected) proximity to other previously identified cluster members or non-members, previous identifications, and star counts.

For the NAN data, though the complete catalog is comparable in size to that from Taurus, we have many more objects with YSO-like colors to consider, and different ancillary data. However, our fundamental approach is similar to that in Taurus. We started with the objects selected in the [3.6] versus [3.6]-[24] $\mathrm{CMD}$, and we folded in several considerations to assess the likelihood of youth and therefore membership in the NAN. In each case, the assessment can be described as an educated statistical guess (e.g., an object selected in the [3.6] versus [3.6]-[24] CMD is statistically more likely than an object not selected in that diagram to be a YSO), but that does not ensure that all YSOs will be selected, nor that all objects that are selected are YSOs. Objects that had no available data for any particular one of these items were unaffected by the lack of such data. (Note that the description here includes forward references to columns in Table 4, which tabulates whether a given YSO candidate met the listed criterion.)

1. If the object appears to the right of the dotted line in Figure 10, e.g., if it is in the space occupied by most YSO candidates in the [3.6] versus [3.6]-[24] CMD, then it is a likely YSO (Column 5). 
Table 3

Complete List of YSOs I. Spitzer Measurements

\begin{tabular}{|c|c|c|c|c|c|c|c|c|c|c|c|c|}
\hline Name & R.A. (J2000) & Decl. (J2000) & 2MASS Name & Alt. Name & Sp. Ty. & Sp. Ty. Src & [3.6] (mag) & [4.5] (mag) & [5.8] (mag) & [8.0] (mag) & [24] (mag) & [70] (mag) \\
\hline $204721.51+441025.1$ & 311.839630 & 44.173649 & $20472150+4410253$ & .. $>$ & $\cdots$ & . & $13.04 \pm 0.06$ & & $12.16 \pm 0.06$ & & $7.52 \pm 0.05$ & . \\
\hline $204736.09+442929.9$ & 311.900391 & 44.491657 & $20473611+4429302$ & & $\ldots$ & $\ldots$ & $10.10 \pm 0.05$ & & $9.88 \pm 0.06$ & & $7.99 \pm 0.05$ & \\
\hline $204744.30+442538.0$ & 311.934601 & 44.427231 & $20474431+4425382$ & . & $\ldots$ & $\ldots$ & $12.51 \pm 0.05$ & & $12.33 \pm 0.06$ & & $7.82 \pm 0.05$ & \\
\hline $204749.74+441128.7$ & 311.957275 & 44.191322 & $20474972+4411295$ & . & .. & $\ldots$ & $13.51 \pm 0.06$ & $12.96 \pm 0.06$ & $12.18 \pm 0.07$ & $10.34 \pm 0.06$ & $6.23 \pm 0.04$ & .. \\
\hline $204753.04+444756.3$ & 311.971039 & 44.798981 & $20475304+4447563$ & ... & $\ldots$ & $\ldots$ & $9.22 \pm 0.05$ & 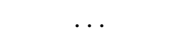 & $8.72 \pm 0.05$ & $\ldots$ & $6.93 \pm 0.05$ & $\cdots$ \\
\hline $204758.30+442059.9$ & 311.992950 & 44.349998 & $20475831+4421000$ & $\ldots$ & $\ldots$ & $\ldots$ & $10.24 \pm 0.05$ & $9.85 \pm 0.05$ & $9.48 \pm 0.05$ & $9.18 \pm 0.06$ & $7.81 \pm 0.13$ & $\cdots$ \\
\hline $204803.57+440529.2$ & 312.014893 & 44.091465 & $20480358+4405295$ & $\cdots$ & $\ldots$ & $\ldots$ & $12.86 \pm 0.06$ & $12.67 \pm 0.06$ & $12.49 \pm 0.07$ & .. & $6.94 \pm 0.06$ & $\cdots$ \\
\hline $204807.74+440003.2$ & 312.032288 & 44.000915 & $20480776+4400032$ & $\cdots$ & $\ldots$ & $\ldots$ & $7.50 \pm 0.05$ & $7.44 \pm 0.05$ & $7.22 \pm 0.05$ & $6.94 \pm 0.05$ & $6.54 \pm 0.04$ & $\cdots$ \\
\hline $204821.48+444114.9$ & 312.089539 & 44.687485 & $20482150+4441150$ & .. & $\ldots$ & $\ldots$ & $12.55 \pm 0.06$ & $12.19 \pm 0.06$ & $11.71 \pm 0.06$ & $11.04 \pm 0.06$ & $6.51 \pm 0.04$ & $\ldots$ \\
\hline $204825.38+442306.5$ & 312.105774 & 44.385151 & $20482536+4423069$ & & $\ldots$ & $\ldots$ & $12.48 \pm 0.06$ & $12.19 \pm 0.06$ & $11.68 \pm 0.06$ & $10.95 \pm 0.07$ & $8.02 \pm 0.13$ & \\
\hline
\end{tabular}

(This table is available in its entirety in a machine-readable form in the online journal. A portion is shown here for guidance regarding its form and content.) 
Table 4

Complete List of YSOs II. YSO Criteria ${ }^{a}$

\begin{tabular}{|c|c|c|c|c|c|c|c|c|c|c|c|c|c|c|c|}
\hline $\begin{array}{c}\text { Name } \\
\text { (1) }\end{array}$ & $\begin{array}{l}\text { Cluster } \\
\text { Name } \\
(2)\end{array}$ & $\begin{array}{c}\text { YSO } \\
\text { Quality } \\
(3)\end{array}$ & $\begin{array}{c}\text { YSO } \\
\text { Class } \\
(4)\end{array}$ & $\begin{array}{c}\text { Sel. } \\
3324 \\
(5)\end{array}$ & $\begin{array}{c}\text { Not Too } \\
\text { Faint } 3324 \\
\text { (6) }\end{array}$ & $\begin{array}{c}\text { Sel. } \\
\text { KK24 } \\
(7)\end{array}$ & $\begin{array}{c}\text { Det@70 } \\
\text { (8) }\end{array}$ & $\begin{array}{c}\text { Picked } \\
\text { G09 } \\
(9)\end{array}$ & $\begin{array}{l}\text { Dist. } \\
\text { (10) }\end{array}$ & $\begin{array}{l}\text { Clust. } \\
\text { (11) }\end{array}$ & $\begin{array}{c}\text { Known } \\
\text { YSO } \\
(12)\end{array}$ & $\begin{array}{l}\text { Sel. } \\
\text { IVI } \\
(13)\end{array}$ & $\begin{array}{c}\text { Sel. } \\
\text { rri } \\
(14)\end{array}$ & $\begin{array}{c}\text { Bright } \\
\mathrm{H} \alpha \\
(15)\end{array}$ & $\begin{array}{l}\text { Sel. } \\
\text { rH } \alpha \text { ri } \\
(16)\end{array}$ \\
\hline $204721.51+441025.1$ & $\ldots$ & $\mathrm{C}$ & Flat & Yes & Yes & No & $\ldots$ & $\cdots$ & No & No & ${ }^{\circ}$ & & & & $\cdots$ \\
\hline $204736.09+442929.9$ & $\ldots$ & $\mathrm{C}$ & III & Yes & Yes & Yes & $\ldots$ & $\ldots$ & No & No & $\ldots$ & $\ldots$ & Yes & $\ldots$ & No \\
\hline $204744.30+442538.0$ & $\ldots$ & $\mathrm{C}$ & II & Yes & Yes & Yes & $\ldots$ & $\ldots$ & No & No & $\ldots$ & $\ldots$ & $\ldots$ & $\ldots$ & $\ldots$ \\
\hline $204749.74+441128.7$ & $\ldots$ & $\mathrm{B}$ & I & Yes & Yes & No & $\ldots$ & Yes & No & No & $\ldots$ & $\ldots$ & $\ldots$ & $\ldots$ & $\ldots$ \\
\hline $204753.04+444756.3$ & $\cdots$ & $\mathrm{C}$ & III & Yes & Yes & Yes & $\cdots$ & $\cdots$ & No & No & $\cdots$ & $\ldots$ & $\ldots$ & $\cdots$ & $\cdots$ \\
\hline $204758.30+442059.9$ & $\ldots$ & B & II & Yes & Yes & Yes & $\ldots$ & Yes & No & No & $\ldots$ & $\ldots$ & $\ldots$ & $\ldots$ & $\ldots$ \\
\hline $204803.57+440529.2$ & $\ldots$ & A & II & Yes & Yes & Yes & $\ldots$ & $\ldots$ & No & No & $\ldots$ & $\ldots$ & Yes & $\ldots$ & Yes \\
\hline $204807.74+440003.2$ & $\ldots$ & A & III & Yes & Yes & Yes & $\ldots$ & No & No & No & $\ldots$ & $\ldots$ & Yes & $\ldots$ & No \\
\hline $204821.48+444114.9$ & $\ldots$ & A & II & Yes & Yes & Yes & $\ldots$ & Yes & No & No & $\ldots$ & $\ldots$ & No & $\ldots$ & No \\
\hline $204825.38+442306.5$ & $\ldots$ & B & II & Yes & Yes & Yes & $\ldots$ & Yes & No & No & $\ldots$ & $\ldots$ & $\ldots$ & $\ldots$ & $\cdots$ \\
\hline
\end{tabular}

Notes.

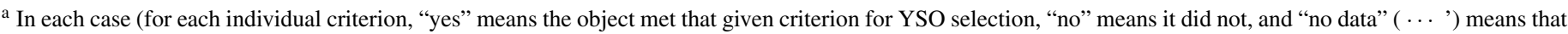

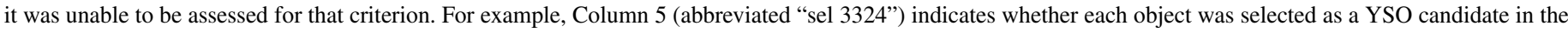

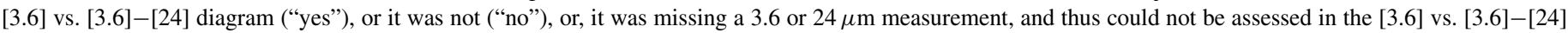
diagram $(\cdots)$. The column definitions are listed in Section 4.2 above.

(This table is available in its entirety in a machine-readable form in the online journal. A portion is shown here for guidance regarding its form and content.)

2. The fainter the object is in [3.6], the more likely that it is a galaxy even if it is red (Column 6).

3. If the object is also selected as bright and red (e.g., a likely YSO) in the $K_{\mathrm{s}}$ versus $K_{\mathrm{s}}-$ [24] CMD, then it is more likely to be a YSO, but if those data $\left(K_{\mathrm{s}}\right.$ and [24]) are available for the target and it is not in the bright and red regime, then it is less likely to be a YSO (Column 7).

4. If an object is detected at $70 \mu \mathrm{m}$, it is more likely to be a YSO, because the $70 \mu \mathrm{m}$ survey is effectively less sensitive than the $24 \mu \mathrm{m}$ survey (e.g., reaches only the brighter excess objects in the NAN-see Figures 10 and 12) (Column 8).

5. If the object was found via the IRAC-based method used in G09, then it is more likely to be a YSO; if it could have been found using that method (e.g., observations exist at all IRAC bands) but was rejected, then it is less likely to be a YSO (Column 9).

6. Because the complete map we have extends past the limits of the bright nebulosity and presumably past the edge of the molecular cloud, if the object was closer to the middle of the nebula, e.g., within $\sim 1^{\circ}$ radius of the approximate center $(\alpha, \delta=313.752,44.231$ or $20: 55: 00.5,+44: 13: 52)$, then it is more likely to be a YSO than if it is further away (Column 10).

7. Because YSOs are more likely to be clustered, if the candidate was in a cluster (see discussion in Section 6.1 below), it is more likely to be a YSO than if it is not in a cluster (Column 11).

8. If an object appeared in the literature as a previously identified YSO using any wavelength, it is substantially more likely to be a legitimate YSO. Similarly, if it was previously identified as a likely contaminant, it is substantially less likely to be a legitimate YSO (Column 12).

9. In the $I_{\mathrm{C}}$ versus $V-I_{\mathrm{C}} \mathrm{CMD}$, if the object appears substantially above the zero-age main sequence (ZAMS), close to where most of the previously identified YSOs are found, then it is more likely to be a YSO; if the object appears below this (or below the ZAMS itself) it is less likely to be a YSO, at least one belonging to the NAN, unless dominated by scattered light at optical wavelengths. (column 13)

10. Similarly, in the IPHAS $r^{\prime}$ versus $r^{\prime}-i^{\prime} \mathrm{CMD}$, if the object appears substantially above the ZAMS (assuming no reddening), near where most of the previously identified YSOs are found, then it is more likely to be a YSO; if it appears below this (or below the ZAMS itself), it is less likely to be a YSO (Column 14).

11. If the object was already identified as a bright $\mathrm{H} \alpha$ source in IPHAS (Witham et al. 2008; Column 15), or appears in the $r^{\prime}-H \alpha$ versus $r^{\prime}-i^{\prime}$ diagram as substantially above the unreddened main-sequence locus (column 16), it is more likely to be a YSO; if the object is below this, it is less likely to be a YSO.

Each object was individually initially assessed resulting in a distribution of confidence levels, which we arbitrarily bin into three groups $-\sim 700$ in grade A, $\sim 500$ in grade B, and $\sim 200$ in grade $\mathrm{C}$. However, there is still more information that can be included.

\subsection{Further Considerations}

Even with all of the Spitzer+ancillary data above, there are still sources left in the pool of possible candidates that are unlikely to be true YSOs. The SED for each YSO was examined by hand, along with each object's status for each item from the prior semi-automatic step. Objects that were too bright (likely foreground and/or AGB stars) or were clearly reddened photospheres (likely background giants) or had SEDs resembling known extragalactic objects or had very low significance excesses or very sparse data (e.g., not many measurements beyond 3.6 and $24 \mu \mathrm{m})$ were removed from the list ( 300 objects) or downgraded in confidence level ( $\sim 100$ objects). (See, e.g., Rebull et al. 2010 for discussion of similar extragalactic and very bright sources.)

Similarly, there are sources that need to be added to the list of candidates. The color cut in the [3.6] versus [3.6]-[24] diagram above summarily rejected sources in the region of this diagram occupied by SWIRE galaxies, but it is known that YSOs can appear in this portion of the diagram. As discussed above (Section 3), the extragalactic contamination is likely to be low in the NAN region, and moreover, where there is very high $A_{V}$, such as in the Gulf of Mexico cluster (see Section 6.2 below), it is unlikely that sources of such red color are extragalactic contaminants. Similarly, there are sources where there is a 


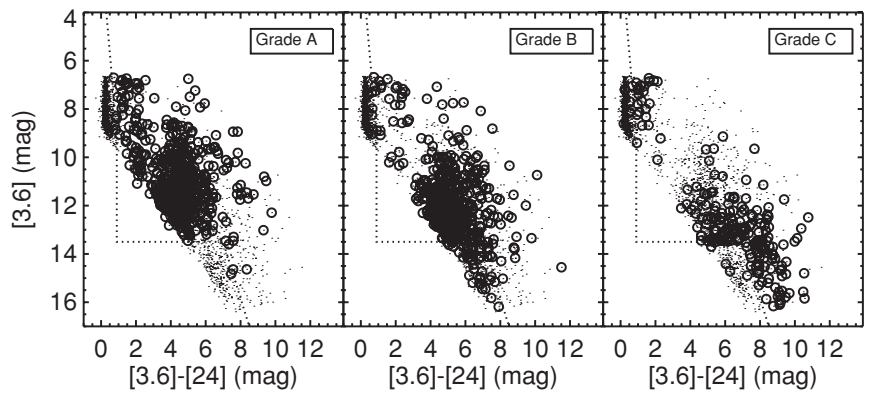

Figure 13. [3.6] vs. [3.6] - [24] plot, with final grade A, B, and C YSO candidates indicated. There are 511 grade $\mathrm{A}, 523$ grade $\mathrm{B}$, and 252 grade $\mathrm{C}$ sources. The grade is listed in Table 4 for each object.

$24 \mu \mathrm{m}$ measurement, but the $3.6 \mu \mathrm{m}$ measurement is missing due to source confusion or reddening or other similar issues; these objects could not be selected in the [3.6] versus [3.6]-[24] diagram but are unlikely to be background galaxies. There are several $(\sim 60)$ sources like this in the NAN; we have added these sources as grade B or C confidence YSO candidates and indicated them as such in the tables below.

\subsection{Complete List of MIPS-selected YSO Candidates}

The complete list of 1286 MIPS-selected YSO candidates appears in Table 3, along with Spitzer measurements and, if available, prior identifications and spectral types. Table 4 repeats the entire list, along with their properties as derived here, including the assigned cluster membership, if applicable, the final YSO quality flag (grades A/B/C), the YSO class (see Section 5.2), and which of the above YSO criteria a given object met. In each case (for each individual criterion), "yes" means the object met that given criterion for YSO selection, "no" means it did not, and "no data" (" ... ") means that it was unable to be assessed for that criterion. For example, Column 5 (abbreviated "sel 3324") indicates whether each object was selected as a YSO candidate in the [3.6] versus [3.6]-[24] diagram ("yes"), or it was not ("no"), or, it was missing a 3.6 or $24 \mu \mathrm{m}$ measurement, and thus could not be assessed in the [3.6] versus [3.6]-[24] diagram (" ... "). The column definitions are listed in Section 4.2 above.

As in Taurus, based on the criteria above (Sections 4.2 and 4.3), we have assigned grades to each source to reflect the confidence in its identification as a YSO. There are in the end 511 grade $A, 523$ grade $B$, and 252 grade $C$ (with a subset of 23 of those being marked "C-"). The highest confidence YSO candidates generally have at least $\sim 5$ or 6 of the criteria listed above in their favor (yeses in Table 4), and the lowest confidence have $\sim 3$. The cumulative YSO candidate list is plotted in Figure 13 separated by grade. The faintest ones are overall more likely to be contaminants, the clump of bright grade $\mathrm{C}$ candidates is some of the ones that were manually downgraded (in Section 4.3) due to less significant excesses, and the ones in the region occupied by SWIRE galaxies are the ones manually promoted in Section 4.3. For the analysis in the rest of this paper, all of the candidates are considered as one large set.

Table 5 summarizes a variety of statistics on the YSO sample selected here, that selected by G09 methodology, and the initial sample of YSOs from the literature. Note that, because our MIPS survey is shallower than our IRAC survey for relatively blue objects, $\sim 40 \%$ of the objects selected using the G09 IRAC methodology do not have MIPS counterparts. The objects

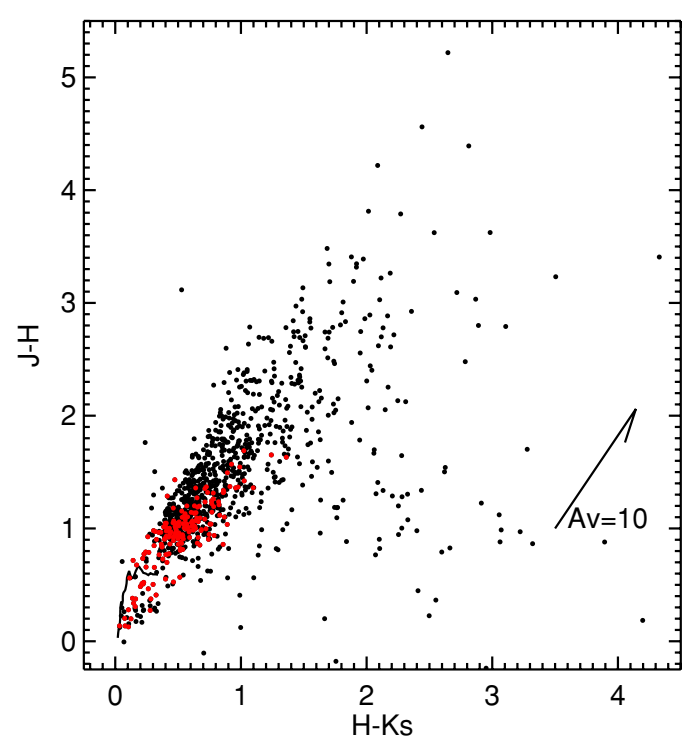

Figure 14. $J-H$ vs. $H-K_{\mathrm{s}}$ for the $80 \%$ of our YSO candidates with such data available. Red points indicate that the objects are also detected at $V I_{C}$; these are generally the less-embedded objects. A reddening vector is indicated, as is the main-sequence locus (line in lower left).

selected by G09/IRAC but not recovered by our MIPS-based selection are listed in Appendix C. The objects found by our MIPS-based selection but not by G09/IRAC, in most cases, are those with small or no excesses at IRAC bands but clear excesses at $24 \mu \mathrm{m}$ (and sometimes longer wavelengths as well). Previously identified YSOs from the literature that were not recovered (via IRAC or MIPS selection) do not have an infrared excess that we could measure, and they appear in Appendix $\mathrm{C}$ as well. These objects may or may not be legitimate NAN members; we cannot determine this from our data.

In certain contexts below, the objects that were IRAC-selected but not recovered by MIPS, and those previously identified YSOs not recovered by either IRAC or MIPS, may be included in the discussion with the MIPS-selected objects. These instances are all clearly noted.

\section{PROPERTIES OF THE ENSEMBLE OF YSO CANDIDATES}

\subsection{Optical and Near-IR Properties}

We have $V I_{\mathrm{C}}$ data for $14 \%$ and $J H K_{s}$ for $\sim 80 \%$ of our YSO candidates. The $V I_{\mathrm{C}}$ objects tend to be the brighter and/or less embedded of the YSO candidate sample.

Figure 14 shows the $J-H$ versus $H-K_{\mathrm{s}}$ color-color diagram for the candidates. The subset of objects with $V I_{\mathrm{C}}$ data are indicated. As suggested by earlier figures, there is generally high $A_{V}$ in the direction of the NAN. $A_{V} \sim 10-20$ are common. Near-infrared evidence for the disks selected by the Spitzer color criteria is apparent.

\subsection{YSO Classes}

In the spirit of Wilking et al. (2001), we define the near- to mid-IR slope of the SED, $\alpha=d \log \lambda F_{\lambda} / d \log \lambda$, where $\alpha>0.3$ for a Class I, 0.3 to -0.3 for a flat-spectrum source, -0.3 to -1.6 for a Class II, and $<-1.6$ for a Class III. For each of the YSO candidate objects in our sample, we performed a simple ordinary least-squares linear fit to all available photometry (just detections, not including upper or lower limits) between 2 and 
Table 5

Statistics of YSO Candidate Sample

\begin{tabular}{|c|c|c|}
\hline Item & Number & Notes \\
\hline Number of previously known YSOs in database (hereafter "prev. known YSOs") & 201 & \\
\hline Number prev. known YSOs with any IRAC measurement & 197 & \\
\hline Number of prev. known YSOs with IRAC measurements in all four bands & 151 & \\
\hline Number of prev. known YSOs with [24] measurement & 108 & $54 \%$ of prev. known YSOs \\
\hline Number of YSO candidates identified by G09 method (hereafter "G09 YSOs") & 1750 & \\
\hline Number of G09 YSOs with [24] measurement & 1012 & $58 \%$ of G09 YSOs \\
\hline Number of YSO candidates identified here & 1286 & \\
\hline Number of prev. known YSOs recovered as YSOs by G09 & 77 & \\
\hline Number of prev. known YSOs recovered as YSOs here & 80 & \\
\hline Number of G09 YSOs recovered as YSOs here & 954 & $94 \%$ of G09 YSOs with M24 \\
\hline Number of prev. known YSOs recovered as YSOs by G09 and here & 70 & \\
\hline Number of prev. known YSOs not recovered as YSOs by G09 or here & 114 & Possible non-members? \\
\hline Number of G09 YSOs not recovered as YSOs here & 796 & \\
\hline Number of G09 YSOs with [24] measurements not recovered as YSOs here & 58 & \\
\hline
\end{tabular}

$24 \mu \mathrm{m}$, inclusive. Note that errors on the infrared points are so small as to not affect the fitted SED slope. The precise definition of $\alpha$ can vary, resulting in different classifications for certain objects. Classification via this method is provided specifically to enable comparison within this paper via internally consistent means. Note that the formal classification puts no lower limit on the colors of Class III objects (thereby including those with SEDs resembling bare stellar photospheres, and allowing for other criteria to define youth). By searching for IR excesses, we are incomplete in our sample of Class III objects.

Figure 15 shows a histogram of the SED slopes, calculated as described, for the MIPS-selected sample, the IRAC-selected sample not recovered by the MIPS-based search, and the complete Spitzer-selected sample (IRAC-selected plus MIPSselected). The objects not recovered by MIPS in general have more negative slopes, because the objects that are not detected by MIPS are the ones that are the faintest in the MIPS bands, e.g., the ones whose SEDs are falling through the IRAC bands. The IRAC-selected sources with steeply rising SEDs (suggesting that they should have been detected at $24 \mu \mathrm{m}$ ) that are not recovered by our MIPS selection are either off the edge of the MIPS map or saturated at MIPS bands.

We repeated the spectral index analysis with slopes fit to just available points between 2 and $8 \mu \mathrm{m}$ (e.g., ignoring any points at $24 \mu \mathrm{m}$ and longer). The peaks of the histograms of the MIPSand IRAC-selected samples move closer together, but the MIPSselected peak is still to the right of the IRAC-selected peak, and the relative fractions of sources in the Class bins do not change.

The MIPS-selected sample is nearly half Class IIs (48\%). Including the IRAC-selected sample as well, the fraction of Class IIs among the selected IR-excess objects rises to $56 \%$. For either sample, there are about $8 \%$ Class IIIs, and the remaining objects are evenly split between Class Is and flat objects (with an additional $2 \%$ where there are too few points to calculate a slope). The relative numbers are quite comparable to the fraction of such objects found in Serpens by Harvey et al. (2007a) or Perseus (Rebull et al. 2007), suggesting that these regions are all comparable in the status of the circumstellar material and hence possibly their age.

\subsection{Projected Location of the YSO Candidates}

Figure 16 shows the projected locations of all of the YSO candidate sources selected using our MIPS-based selection. Several clusters of objects can be seen by eye here; we will consider clustering formally in the next section. There is also

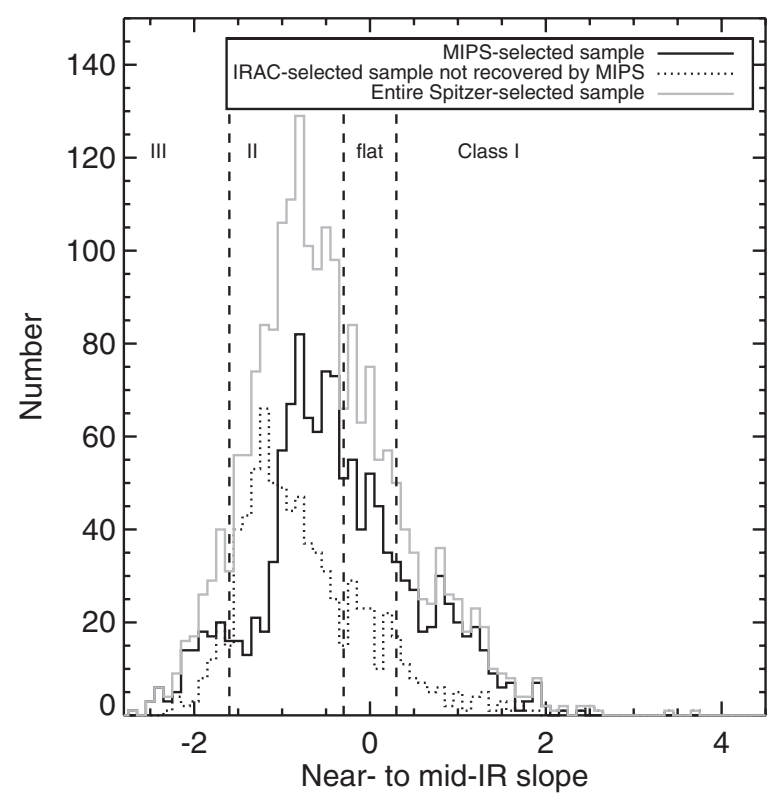

Figure 15. Histogram of SED slopes, calculated as described in the text, for the MIPS-selected sample (solid line) and the IRAC-selected sample not recovered by MIPS (dotted lines). The entire Spitzer selected sample (MIPS and IRAC selected) is the gray solid line, e.g., the sum of the other two histograms. Dashed lines denote the adopted divisions between Class I, flat, Class II, and Class III. Note that since we are identifying YSOs by their IR excess, our Class III sample is defined to be incomplete. The bulk of our Spitzer-selected sample of YSO candidates have SED slopes consistent with those of Class II YSOs.

a distributed population. The fact that there is a substantial distributed population here is consistent with results from many other Spitzer studies of star-forming regions, where substantial numbers of YSO candidates are found far from the canonical centers of star formation.

In Figure 2, we saw large spatial variation in the $24 \mu \mathrm{m}$ surface brightness due to the ISM, which renders the limiting flux density a function of position in the image. Similar structures can be seen at the IRAC bands (see G09). It is difficult to assess if this has affected the apparent distribution of the selected YSO candidates. The distribution of YSOs is not uniform, and it is expected based on physical arguments that the distribution of YSOs will be coupled to the ISM, especially at these young ages. We examined the distribution of YSO candidates (and all $24 \mu \mathrm{m}$ sources) by plotting them on top of the $24 \mu \mathrm{m}$ image. We do not see a very strong effect of the ISM brightness on our YSO 


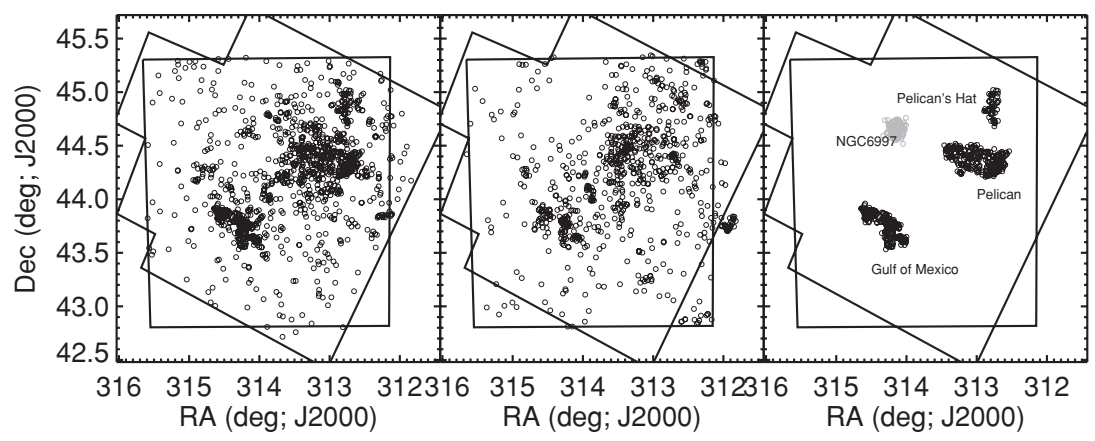

Figure 16. Projected locations of (left) entire set of MIPS-selected YSO candidates, (middle) set of IRAC-selected YSOs not recovered by the MIPS-based selection, and (right) clusters as discovered in the MIPS-based distribution discussed further below. In all three panels, the polygons from Figure 1 are reproduced, indicating the approximate IRAC and MIPS coverage; note also that in all three panels, only hollow circles are used as symbols-black portions come from overlapping symbols. In the right-most panel, NGC 6997 is represented in gray because it is actually a background (or possibly foreground) object, it is defined in the literature (as opposed to defined on the basis of YSO candidates here), and primarily consists of non-IR excess sources. It is included here for reference; see discussion in Appendix B.1.

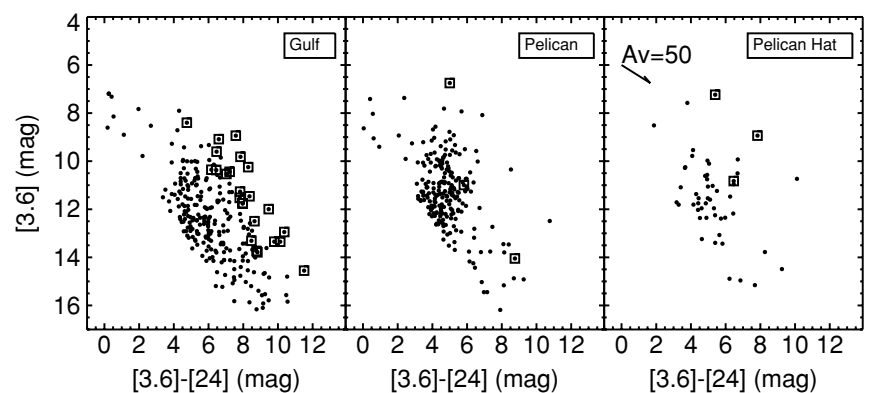

Figure 17. [3.6] vs. [3.6] - [24] plots for YSO candidates in three clusters located in the NAN complex; compare to Figure 10 or 11 . An additional box around a point indicates detection at $70 \mu \mathrm{m}$. The Pelican's Hat cluster is the least populous. There is a wide range of colors found in each of these clusters. The distribution of objects in the Pelican cluster is more clumped than that from the Gulf of Mexico, perhaps due to the more substantial reddening in the Gulf.

candidate distribution. Qualitatively, we note that the bright "streaks" in the center of the map have few YSO candidates, and also fewer $24 \mu \mathrm{m}$ sources in general; the even brighter ISM in the region of the Pelican cluster hosts many YSO candidates. We conclude that the ISM surface brightness is not a dominant effect in the structure seen in the YSO candidate distribution. However, many of the YSO candidates are projected against the regions of highest extinction (which correlates well with the MIPS surface brightness), with concentrations near the Gulf of Mexico and the greater Pelican region.

As discussed above, there are a significant number of IRACselected YSO candidates that are not recovered by our MIPSbased selection, and these appear in the second panel of Figure 16. Clustering similar to the left panel can be seen by eye, even in this residual IRAC-only selection.

\section{CLUSTERS OF YSO CANDIDATES WITHIN THE NAN COMPLEX}

\subsection{Defining the Clusters}

G09 discussed in some detail a formal definition of clustering, using a Kernel method to estimate the density of YSO candidates (note that not the density of sources in the entire catalog) as a function of $\alpha, \delta$. Using the same formalism, we computed a YSO density map of our final set of MIPS-selected YSO candidates. There are three main clusters that can be identified out of this sample. These three clusters appear in the rightmost panel of Figure 16. (A background cluster is also indicated in that figure for reference; see discussion in Appendix B.1.)

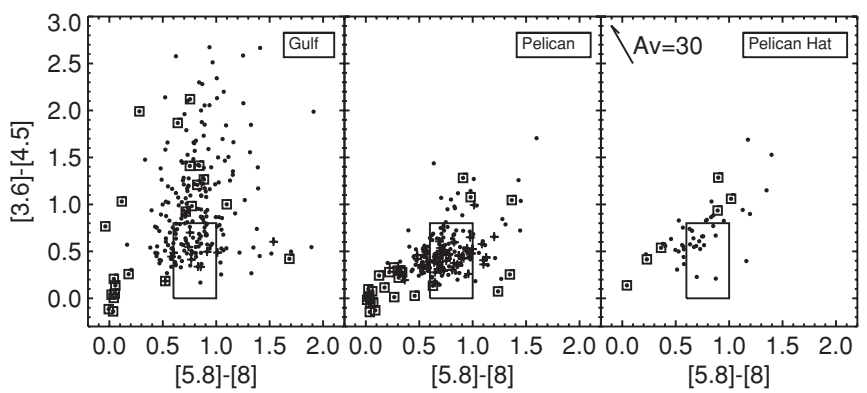

Figure 18. IRAC color-color diagram for MIPS-selected YSO candidates in three clusters located in the NAN complex. Objects previously identified as YSOs in the literature and recovered here are indicated by a + sign; an additional box around a point denotes objects identified here but not in G09. Note that YSO candidates identified in G09 but not here do not appear in these plots. The large box comes from Allen et al. (2004) and indicates the unreddened location of likely Class II objects. As in Figure 17, there is a wide range of colors of YSO candidates, and the Gulf of Mexico cluster seems to be the most reddened.

We name these three clusters based on their position with respect to the nebular features-the Gulf of Mexico cluster is located in the Gulf of Mexico region of the North American Nebula; the Pelican cluster overlaps with the Pelican's neck, head, and beak; and the third cluster appears above the Pelican's head, hence "Pelican's Hat." We discuss the properties of these clusters in turn below. Cluster membership is indicated for individual objects in Table 4. Ensemble properties are summarized in Table 6; plots of [3.6] versus [3.6]-[24] are in Figure 17 and plots of [3.6]-[4.5] versus [5.8]-[8] are in Figure 18.

Because clustering is considered in the selection of YSO candidates above (Section 4.2), and because some sources were added by hand in regions of high YSO density (see Section 4.3), identifying and defining clusters are somewhat of a recursive problem, with the potential for biased results. We also ran the cluster identification algorithm on the initial and least biased sample of [3.6] versus [3.6]-[24] selected objects (Section 4.1). The three clusters were unambiguously recovered even in this sample, so we conclude that they are independent of the details of our YSO selection algorithm. (We note that heavily reddened $\mathrm{K}$ and $\mathrm{M}$ giants located behind a very dense dust cloud could appear to be clustered YSOs; see, e.g., Straižys \& Laugalys 2008a.)

In the left and middle panels of Figure 16, there are additional smaller clumpings of sources to which one's eye is drawn, most notably a clump on the far west of the distribution. It has a 


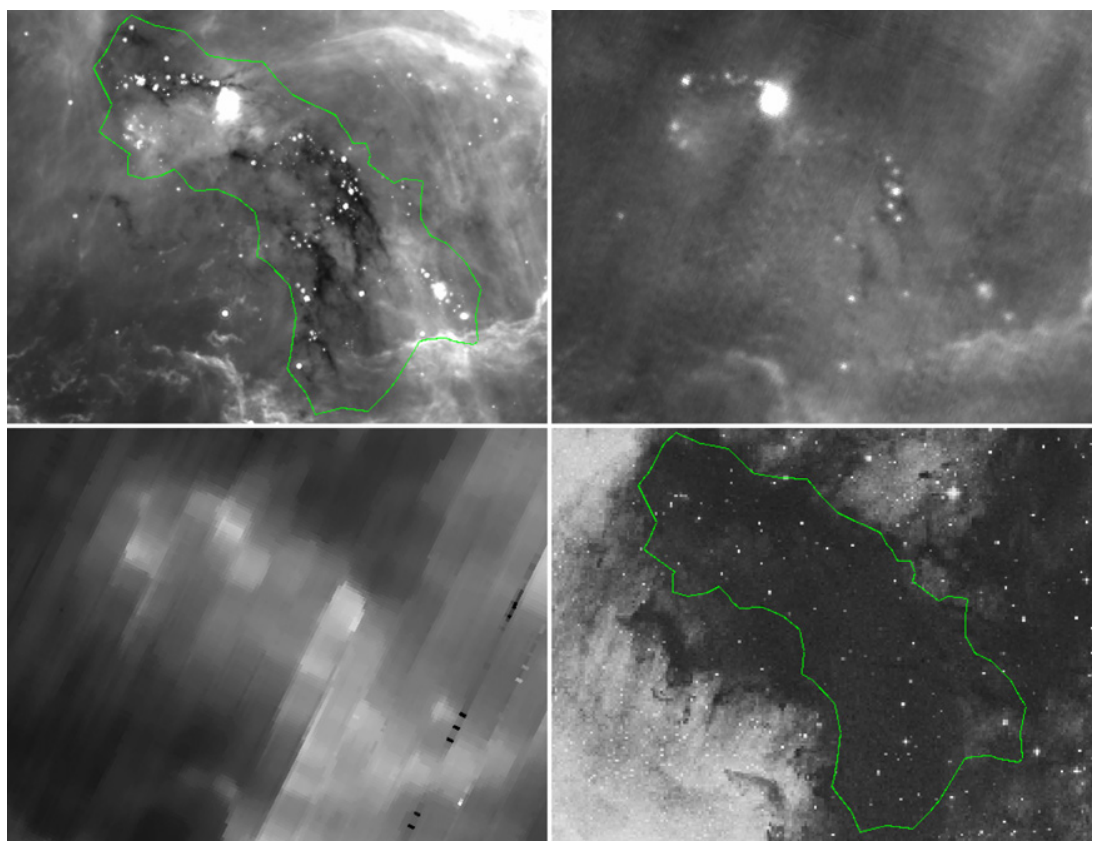

Figure 19. Mosaic of Gulf of Mexico at $24 \mu \mathrm{m}$ (top left), $70 \mu \mathrm{m}$ (top right), $160 \mu \mathrm{m}$ (lower left), and, for comparison, POSS-red (lower right). The gray-scale colors correspond to a histogram-equalized stretch of surface brightnesses in each case. The contour (plus YSO candidacy) defines cluster membership and is essentially the $A_{V}=10$ contour from the Cambrésy et al. (2002) extinction map.

(A color version of this figure is available in the online journal.)

Table 6

Statistics of MIPS-selected YSO Candidate Detections in Clusters

\begin{tabular}{lccc}
\hline \hline Item & Gulf of Mexico & Pelican & Pelican's Hat \\
\hline $24 \mu \mathrm{m}$ & 283 & 247 & 51 \\
$70 \mu \mathrm{m}$ & 29 & 3 & 3 \\
$24 \mu \mathrm{m} \& 70 \mu \mathrm{m}$ & 29 & 3 & 3 \\
$24 \mu \mathrm{m} \&$ ANY IRAC band & 269 & 233 & 51 \\
$70 \mu \mathrm{m}$ \& ANY IRAC band & 26 & 3 & 3 \\
$24 \mu \mathrm{m}$ \& ALL IRAC bands & 240 & 216 & 48 \\
$70 \mu \mathrm{m}$ \& ALL IRAC bands & 22 & 2 & 3 \\
$24 \mu \mathrm{m}$ \& ANY 2MASS band & 162 & 210 & 43 \\
$24 \mu \mathrm{m}$ \& ANY IPHAS band & 36 & 143 & 5 \\
$24 \mu \mathrm{m}$ \& $V$ or $I_{c}$ or $r^{\prime}$ or $i^{\prime}$ & 36 & 148 & 5 \\
Approximate area (deg $\left.{ }^{2}\right)$ & 0.105 & 0.135 & 0.051 \\
\hline Class I sources & $109(39 \%)$ & $31(13 \%)$ & $15(29 \%)$ \\
Flat sources & $91(32 \%)$ & $33(13 \%)$ & $20(39 \%)$ \\
Class II sources & $68(24 \%)$ & $157(64 \%)$ & $16(31 \%)$ \\
Class III sources (incomplete) & $4(1 \%)$ & $12(5 \%)$ & $0(0 \%)$ \\
Insufficient data for class & $11(4 \%)$ & $14(6 \%)$ & $0(0 \%)$ \\
\hline
\end{tabular}

linear string of YSOs in the MIPS-selected sample and a slightly offset clump of sources on the edge of the IRAC map from the IRAC-selected YSOs. Neither of these groupings appear in the clustering analysis above.

There are legitimate YSOs here outside of the clusters, notably the outbursting Class I object PTF10nvg (Covey et al. 2011), which is south of the Pelican cluster.

Parts of two of the clusters described here (the Gulf of Mexico and the Pelican) have been previously identified in the literature (based primarily but not exclusively on $\mathrm{H} \alpha$ ); our data suggest expanded spatial definitions (and far more members) for these clusters. The Pelican's Hat cluster is an entirely new discovery based on the MIPS data. In Appendix B, we discuss additional clusters from the literature that are sufficiently unremarkable in the MIPS maps that they are mentioned here solely for completeness.
The SEDs presented below are all in $\log \lambda F_{\lambda}$ in cgs units (erg s${ }^{-1} \mathrm{~cm}^{-2}$ ), against $\log \lambda$ in $\mu \mathrm{m}$.

\subsection{Gulf of Mexico}

The "Gulf of Mexico" region is the area of highest extinction in the NAN complex; the Cambrésy et al. (2002) extinction map peaks at $A_{V} \sim 30$, and is likely a lower estimate to the true extinction due to the fact that it was calculated using near-IR data and is lower spatial resolution than our MIPS data. Herbig (1958) identified several stars bright in $\mathrm{H} \alpha$ at the northernmost part of the cluster we describe below. Cambrésy et al. (2002) find three subclusters within this cluster using 2MASS star counts (in their notation, 1, 2, and 3a/b); G09 also finds three subclusters within this cluster using IRAC.

As can be seen in Figure 19, the image of this region is striking at $24 \mu \mathrm{m}$ (and $8 \mu \mathrm{m}$; not shown), with infrared emission and a filamentary dark cloud. Parts of the cloud are dark at $24 \mu \mathrm{m}$ and even at $70 \mu \mathrm{m}$; this region has the highest surface density of $70 \mu \mathrm{m}$ point sources in the complex. Counterparts at $160 \mu \mathrm{m}$ can be seen as well, though no viable photometry can be extracted from our $160 \mu \mathrm{m}$ data. Many jets are visible at the IRAC bands, especially at the northern end of the cluster.

The cluster membership is defined as discussed above, and is represented as a contour in Figure 19; that contour is very close to the $A_{V}=10$ contour from the Cambrésy et al. (2002) extinction map. Using the G09 definition of candidate (IRAC) YSOs and clustering metrics, this contour is similar to that for 1500 (IRAC-selected) YSO candidates per square degree. It is about $30^{\prime}$ across at its widest part, corresponding to about 4.5 $\mathrm{pc}$ at a distance of $520 \mathrm{pc}$. There are 283 objects seen at $24 \mu \mathrm{m}$ that we define as being part of the cluster (see Table 6), which is $\sim 2500 \mathrm{deg}^{-2}$. Note that some objects not explicitly selected via the color mechanisms in Section 4 above (such as those objects seen only at $24 \mu \mathrm{m}$ ) have been added to the YSO candidate list on the basis of their projected location in this cluster (and 
$24 \mu \mathrm{m}$ flux density). At $70 \mu \mathrm{m}, 10 \%$ of the $24 \mu \mathrm{m}$ sample have counterparts. Most of the objects, $85 \%$, are seen at all four IRAC bands, but $5 \%$ are so embedded that they lack any IRAC counterparts at all.

Figures 17 and 18 show the [3.6] versus [3.6]-[24] and the IRAC color-color diagram for this cluster in context with the other clusters discussed here. The Gulf cluster has by far the most $70 \mu \mathrm{m}$ detections (see Table 6), and the widest range of colors of YSO candidates in both these diagrams. While several objects in the Gulf have extreme IR colors, a major contributing factor to the dispersion in colors is the high $A_{V}$.

Our primary YSO candidate selection mechanism above requires MIPS, and we defined clusters based on the location of those MIPS-selected YSO candidates. But, for relatively blue sources, the IRAC survey is more sensitive than the MIPS survey, and there are most likely legitimate Gulf cluster members not detected at MIPS bands. There are $~ 4100$ IRAC- 1 and IRAC-2 sources without MIPS counterparts within the $A_{V}=10$ contour $\left(\sim 40,000 \mathrm{deg}^{-2}\right.$ compared to an average of $\sim 60,000$ $\mathrm{deg}^{-2}$ over the whole map). Just $\sim 800$ of the objects in this region are detected at all four IRAC bands $\left(\sim 8000 \mathrm{deg}^{-2}\right)$. Of those objects, just 92 IRAC-selected YSO candidates (requiring selection in all four IRAC bands) are unrecovered by our MIPSbased selection and could be legitimate cluster members. There are additional likely YSOs that we list as YSO candidates based on 5.8 or 8 through $70 \mu \mathrm{m}$ properties, but they do not have detections at all four IRAC bands. Such sources without 3.6 or $4.5 \mu \mathrm{m}$ detections would not be selected via the G09 mechanism. The 92 IRAC-selected YSO candidates unrecovered by our MIPS-based selection is the minimum number of additional cluster members. Adding those 92 to the 283 we define (based on MIPS) as the cluster membership, we derive a YSO census of 375 members and a surface density of $\sim 3500 \mathrm{deg}^{-2}$.

To constrain the maximum number of true cluster members, we can look at overall source counts. The number per unit area of four-band IRAC detections (without MIPS detections) on the edges of the map, in regions of much lower $A_{V}$, can be taken as roughly the number of background+foreground counts; these are $\sim 11,000 \mathrm{deg}^{-2}$. Considering those objects with IRAC-1 and 2 detections (but no MIPS-24), there are $~ 70,000$ sources $\mathrm{deg}^{-2}$ on the map edges. It is thus unlikely that all projected IRAConly sources could be cluster members, but the IRAC-selected YSO candidates are far more likely to be members than any given IRAC-only source. Additional observations and analysis are needed to distinguish the true cluster members from the foreground stars.

Of 375 cluster members, just 11 were identified in the literature prior to our Spitzer observations as cluster members (with 303 identified in G09). All of the objects known prior to Spitzer are in the northernmost $\sim 6^{\prime}$ of the cluster, and eight of them are in the northermost $\sim 1.5$ of the cluster, so the size of the cluster is redefined as about 10-100 times larger (in projected area) than previously realized.

Figure 20 shows the projected spatial location of the MIPSselected objects, coded by their SED slope. The Class I and flat objects are more clustered than the Class II and Class III objects. Table 6 lists the relative fractions of Class I, flat, II, and III MIPS-selected YSO candidates in the cluster. If the candidates found using IRAC only are included, the relative fractions are $32 \%, 30 \%, 34 \%$, and $2 \%$, respectively (with $3 \%$ for which no slope can be calculated); the largest increase is in the Class II category, consistent with Figure 15. The dense embedded cluster found in Serpens South (Gutermuth et al. 2008) bears

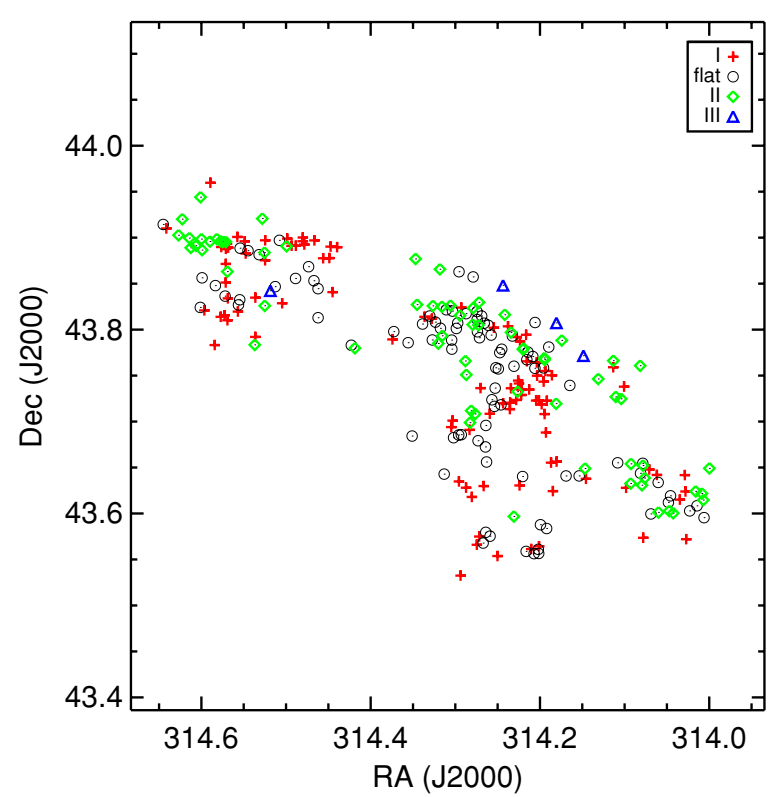

Figure 20. Location of YSOs, color-coded by their SED slope, in the Gulf of Mexico. The Class I and flat objects are more clustered than the Class II and Class III objects.

(A color version of this figure is available in the online journal.)

some morphological similarity to this region. The mechanism used by those authors to select YSOs is very different than what we use, but they find about $60 \%$ Class I+flat objects. In the Gulf, we find that about $62 \%$ of all the YSO candidates are Class I or flat (71\% of just the MIPS-selected sample). Within the NAN complex, the Gulf has the largest fraction of Class I objects. The overwhelming majority of the NAN objects seen in the [24] versus [24]-[70] diagram (Figure 12) are located in the Gulf. We identify this region in the NAN complex to have the most embedded objects (presumably the youngest?), the highest surface density of YSO candidates, and to be where star formation is actively occurring.

Figure 21 shows a sample of 16 SEDs selected out of our proposed cluster membership to represent the range of colors and SED types found in this cluster. One object in this figure is essentially photospheric at all available bands (which may mean that it is a foreground object superimposed on the dense cluster region and erroneously included as a cluster member solely based on its location on the sky), while others in this figure are deeply embedded, appearing only at IRAC+MIPS, not 2MASS or optical. Notably, the object (PTF10qpf) recently identified by Miller et al. (2011) as an FU Ori-like outburst was present in our pre-outburst observations of this cluster.

\subsection{Pelican Cluster}

Herbig (1958) identified a cluster of 12 young objects bright in $\mathrm{H} \alpha$ near IC 5070 (the Pelican Nebula). This cluster was denser than the other clusters Herbig identified in the NAN complex, leading him to conclude that this region hosted the most active region of star formation in the complex. While all of these objects are seen at 2MASS and IRAC bands, none are detected at $24 \mu \mathrm{m}$, and none have [3.6]-[24] excesses; moreover, none are even identified by G09 as IRAC-selected YSOs. There is, however, still a noticeable clumping of Spitzer-identified YSO candidates in this general region; see Figure 16.

Figure 22 shows the Pelican Cluster region in 24, 70, and $160 \mu \mathrm{m}$ as well as a POSS-red image. There are many point 


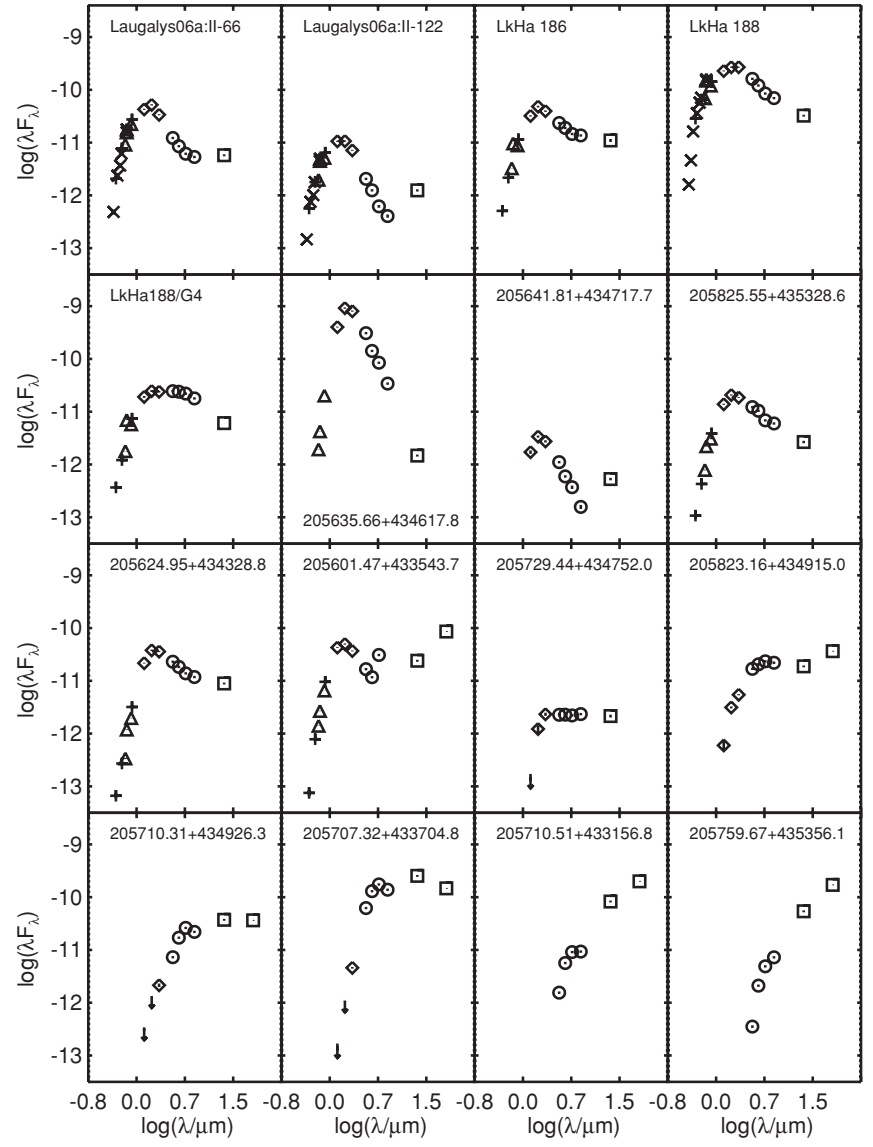

Figure 21. Sample of selected YSO candidate SEDs seen in the Gulf of Mexico cluster; $\lambda F_{\lambda}$ is in erg s $\mathrm{s}^{-1} \mathrm{~cm}^{-2}$, and $\lambda$ is in $\mu \mathrm{m}$. Previously identified YSOs are denoted by their previous name; new objects have IAU-compatible names. Vertical error bars are typically smaller than the points. Symbols denote origin of photometry: $\times$ : Vilnius system, triangles: IPHAS, +: Johnson (from the literature), diamonds: 2MASS, circles: IRAC, squares: MIPS; downwardpointing arrows are upper limits. Note that there is a wide range of SED types, from essentially photospheric (e.g., 205635.66+434617.8) to deeply embedded (e.g., 205759.67+435356.1). sources at $24 \mu \mathrm{m}$; some sources can be seen in the $70 \mu \mathrm{m}$ image, but only three can be identified using our pipeline-most are slightly extended or in regions of extremely bright background. No point sources are discernible at $160 \mu \mathrm{m}$.

The contour defining the cluster membership is shown in Figure 22 as well. Interestingly, unlike the Gulf cluster in Section 6.2 above or in the Pelican's Hat cluster in Section 6.4 below, the definition of the cluster as returned by the formal clustering metrics does not trace an $A_{V}$ contour. Rather, it links two $A_{V} \sim 20$ peaks, seen in the $24 \mu \mathrm{m}$ image as the brightest surface brightness regions. It also overlaps with more than half the projected area of two clusters from Cambrésy et al. (2002), clusters 5 and 6 , though the regions specifically called out by Cambrésy et al. often include regions too bright and nebulous for many MIPS-24 point sources to have been recovered. Similarly, the G09 clusters 5 and 6 are both located within our MIPS-based cluster contour.

Table 6 lists characteristics of this cluster as we now define it. Forty-eight of the 247 cluster members are previously identified as YSOs in the literature, so we have expanded the likely cluster membership by a factor of $\sim 5$. Herbig (1958) identified 13 more $\mathrm{H} \alpha$ sources in this region, but did not explicitly call them out as being part of the IC 5070 sources he identified as a cluster; Ogura et al. (2002) also found several $\mathrm{H} \alpha$ sources here. In contrast to the 12 original Herbig (1958) IC 5070 sources, we detect these Ogura $\mathrm{H} \alpha$ sources at $24 \mu \mathrm{m}$. The fact that nearly $20 \%$ of the cluster membership has already been identified in the literature as young stars is consistent with there being overall lower $A_{V}$ towards this cluster than the Gulf of Mexico and Pelican's Hat clusters identified in this paper. Unlike the Gulf of Mexico cluster, we did not add manually additional objects to the cluster inventory on the basis of $24 \mu \mathrm{m}$ flux density plus clustering. Because the average $A_{V}$ is lower here than in the Gulf, the risk of contamination is higher. However, a high fraction of the IRAC-selected (but not MIPS-recovered) sources are likely to be cluster members, as evidenced by the clustering found in G09 (or, for that matter, Cambrésy et al. 2002).

Figures 17 and 18 and Table 6 show that, although it contains objects of a wide range of colors, the Pelican cluster is almost two-thirds Class II objects; in both Figures 17 and 18, there

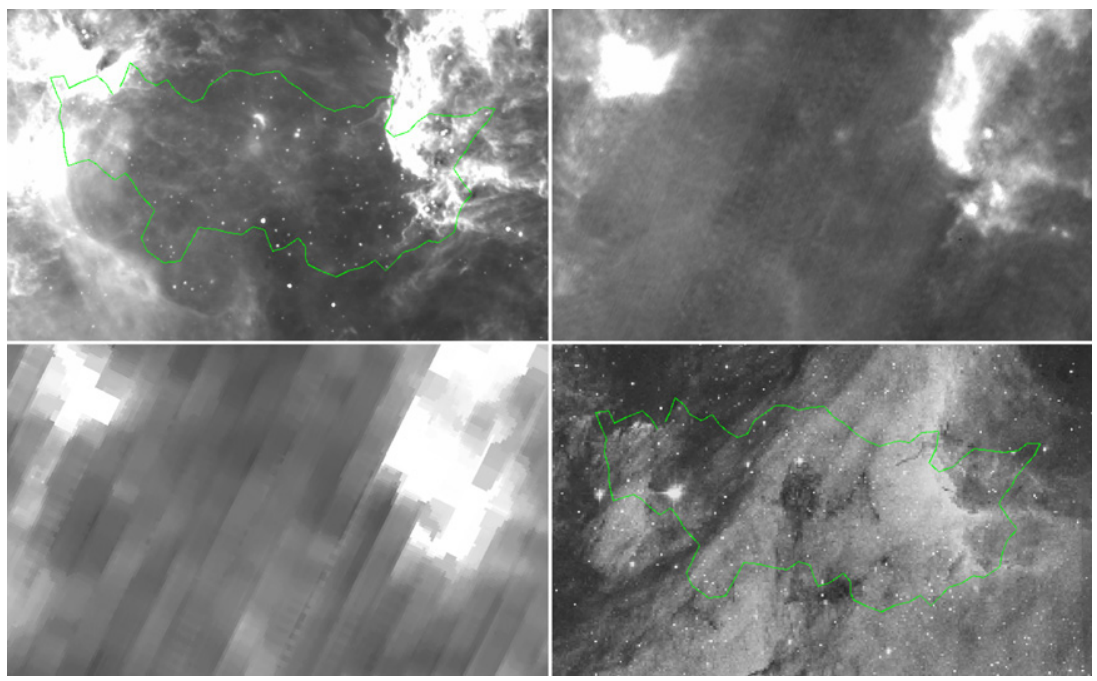

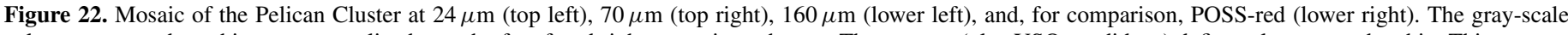

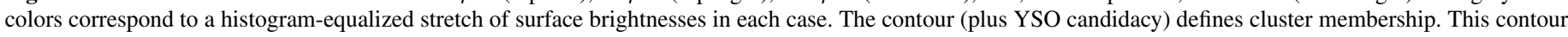
encloses two $A_{V} \sim 20$ peaks (the brightest regions in the $24 \mu \mathrm{m}$ map).

(A color version of this figure is available in the online journal.) 


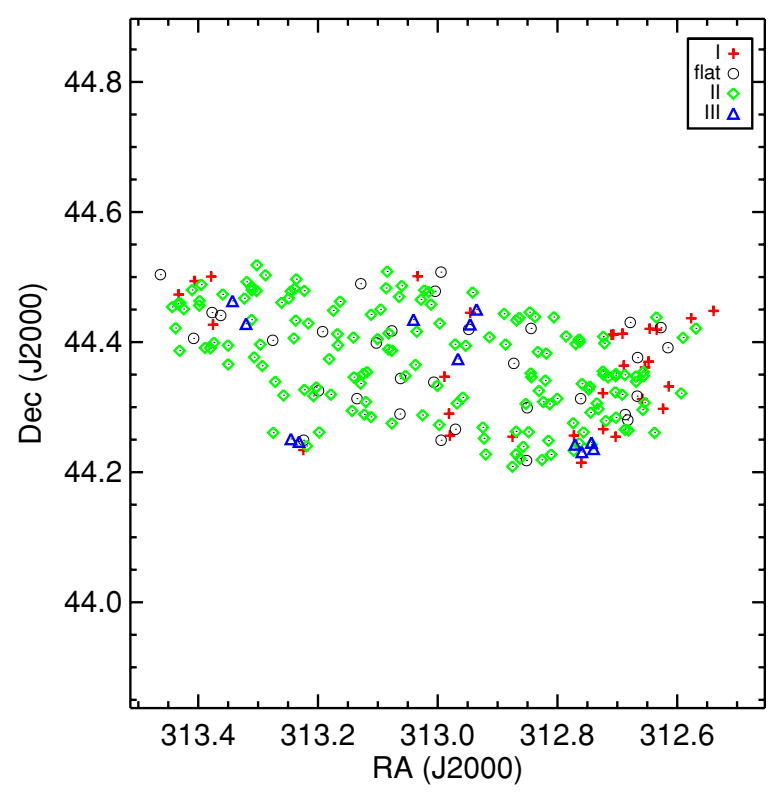

Figure 23. Location of YSOs, color-coded by their SED slope, in the Pelican cluster. The Class I and flat objects are more clustered and located closer to the $A_{V}$ peaks than the Class II and Class III objects.

(A color version of this figure is available in the online journal.)

are regions of higher source density. Among the three clusters under consideration here, this one is significantly more tightly clumped in color space or in class distribution, with a much higher fraction of Class IIs and much lower fraction of Class I or flat objects. This is almost certainly related to the relatively lower $A_{V}$ (and thus less smearing in the color spaces) in the direction of this cluster, but also may be telling us something about the relative degree of embeddedness and possibly the age of this cluster compared to the other two. If this cluster is indeed the oldest of the three clusters discussed here, it has had more time to disperse the surrounding cloud (accounting for the lower $A_{V}$ ) and for the objects to move from their original natal location (accounting for the more spatially dispersed, less tightly clustered locations). Figure 23 shows the (projected) locations of the cluster members. Most of the Class I and flat objects are seen on the east and west edges of the cluster, close to the $A_{V}$ peaks. Figure 24 shows a sample of SEDs selected out of our proposed cluster membership to represent the range of colors and SED types found in this cluster.

\subsection{Pelican's Hat}

North of the Pelican's head, in roughly the same location as would be found a hat on its head, is a previously unidentified dark lane at $24 \mu \mathrm{m}$, similar to but much smaller than the Gulf of Mexico; see Figure 25. This sinuous lane is about $10^{\prime}$ long $(\sim 1.5$ $\mathrm{pc}$ at a distance of $520 \mathrm{pc}$ ), with two bright $24 \mu \mathrm{m}$ sources at the south end, and several other nearby objects detected at $24 \mu \mathrm{m}$. The definition that emerges as part of the cluster formalism above corresponds very closely to an $A_{V} \sim 8$ contour from the Cambrésy et al. (2002) extinction map.

None of the objects found in this region correspond to previously known YSOs or to any other prior identifications. As seen in Figure 25, the region is on the edge of our $70 \mu \mathrm{m}$ map, and so viable data are obtained only for part of the cluster. Texture in the nebulosity (including some nearby patches not formally included in the cluster definition) can be seen. The $160 \mu \mathrm{m}$ image has hints of the same structure.

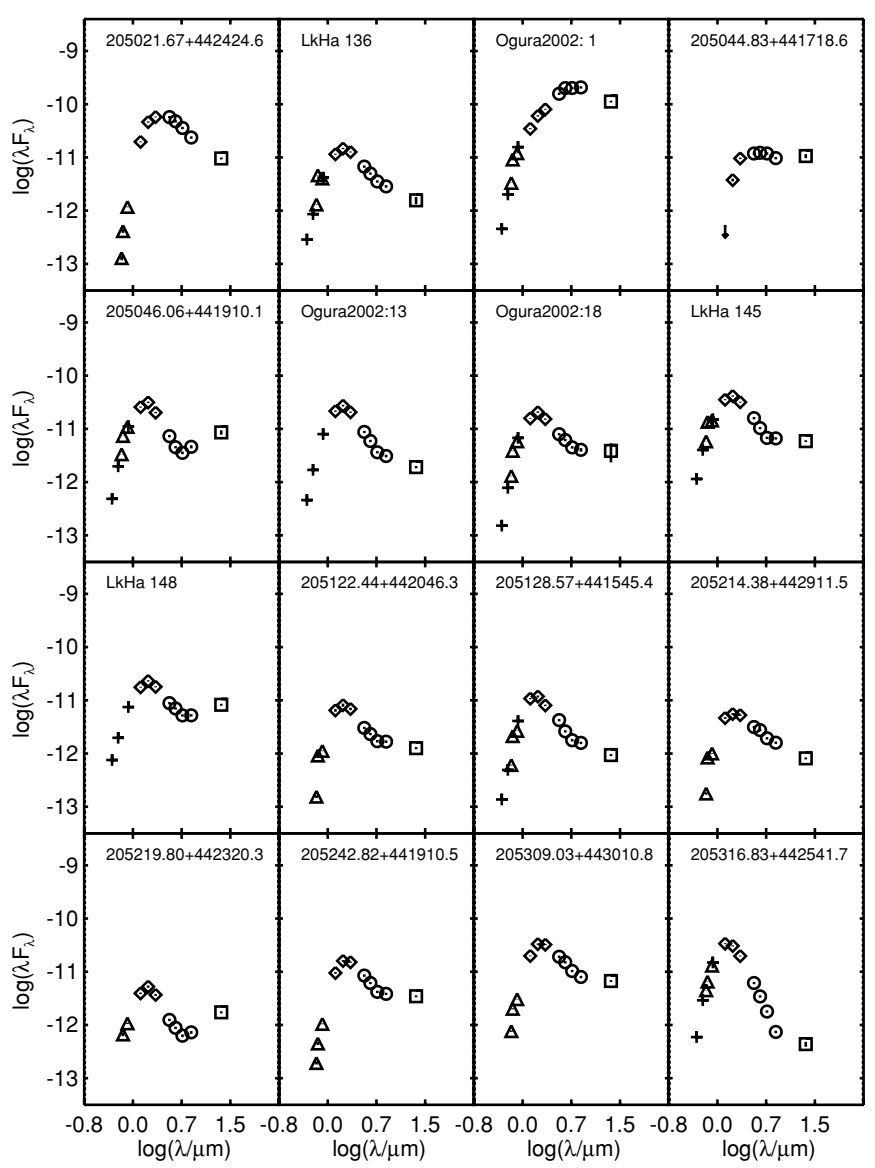

Figure 24. Sample of selected YSO candidate SEDs seen in the Pelican cluster; $\lambda F_{\lambda}$ is in erg $\mathrm{s}^{-1} \mathrm{~cm}^{-2}$, and $\lambda$ is in $\mu \mathrm{m}$. Previously identified YSOs are denoted by their previous name; new objects have IAU-compatible names. Vertical error bars are typically smaller than the points. Symbols denote origin of photometry: $\times$ : Vilnius system, triangles: IPHAS, +: Johnson (from the literature), diamonds: 2MASS, circles: IRAC, and squares: MIPS; downwardpointing arrows are upper limits.

Of the three clusters highlighted in this paper, this is the smallest in projected area, number of sources, and surface density. There are 51 sources we define to be part of this region. Table 6 and Figures 17 and 18 suggest that the cluster may be intermediate in YSO candidate properties, between the Gulf and the Pelican clusters. The relative fraction of objects is greatest in the "flat" class, but within Poisson counting statistics, the relative fractions seen in each of the Class I/flat/Class II/ Class III categories is indistinguishable from the Gulf of Mexico cluster.

Figure 26 shows the projected spatial distribution of YSO candidates; note that there are no Class III objects (whereas the other two clusters have a few), and that each category of object seems to be smoothly distributed. This may be another indication of youth, or this may mean that the actual cluster is larger than our defined area. Figure 27 shows a sample of SEDs selected out of our proposed cluster membership to represent the range of colors and SED types found in this cluster.

There are $\sim 10$ IRAC-selected but not MIPS-recovered YSO candidates in this region, and they are almost all located on the edges of our cluster definition. It is difficult to assess if they should be included in the cluster or not, and we have chosen not to include them.

There are at least two objects nearby but not formally in the cluster definition whose morphology in the $24 \mu \mathrm{m}$ image 


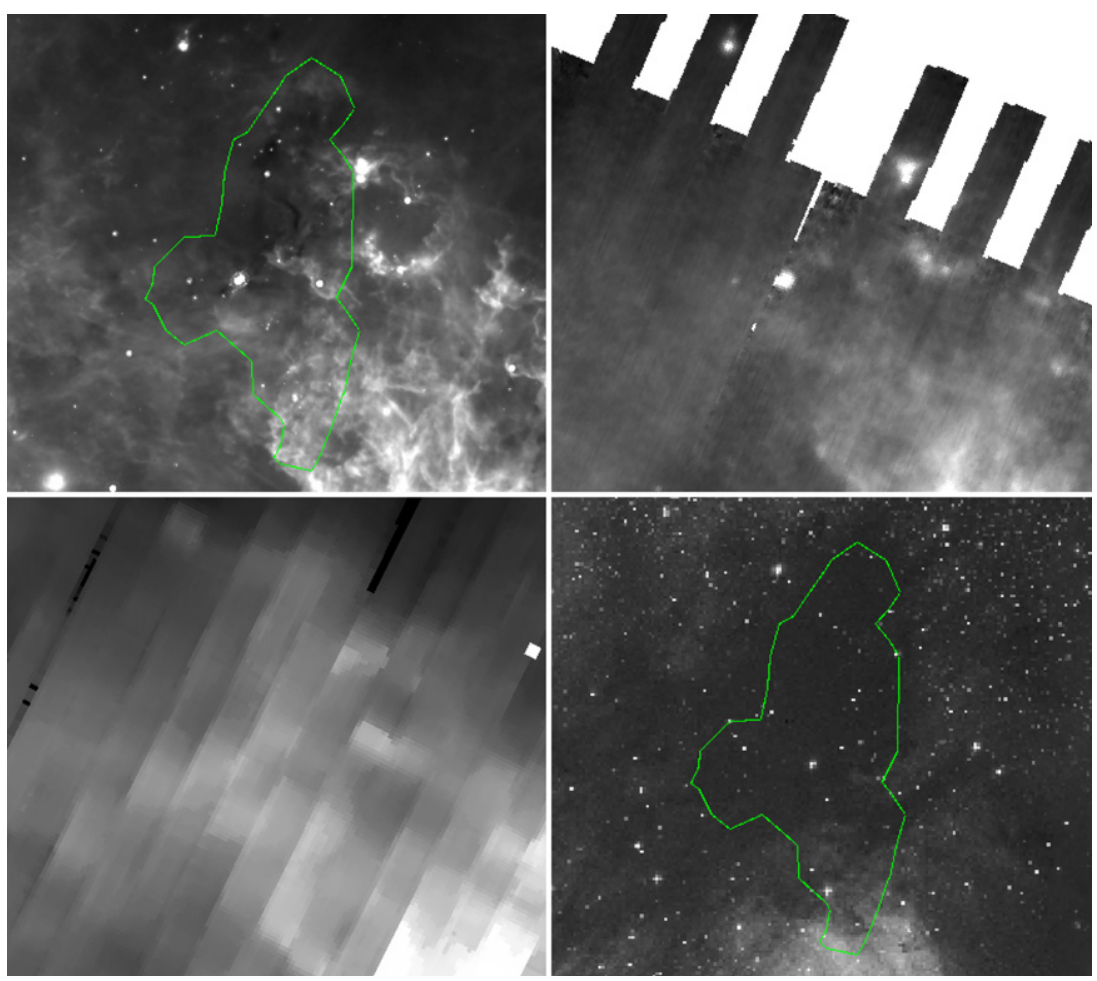

Figure 25. Mosaic of the Pelican's Hat Cluster at $24 \mu \mathrm{m}$ (top left), $70 \mu \mathrm{m}$ (top right), $160 \mu \mathrm{m}$ (lower left), and, for comparison, POSS-red (lower right). The gray-scale colors correspond to a histogram-equalized stretch of surface brightnesses in each case. The contour (plus YSO candidacy) defines cluster membership. This contour is essentially an $A_{V}=8$ contour from the Cambrésy et al. (2002) extinction map.

(A color version of this figure is available in the online journal.)

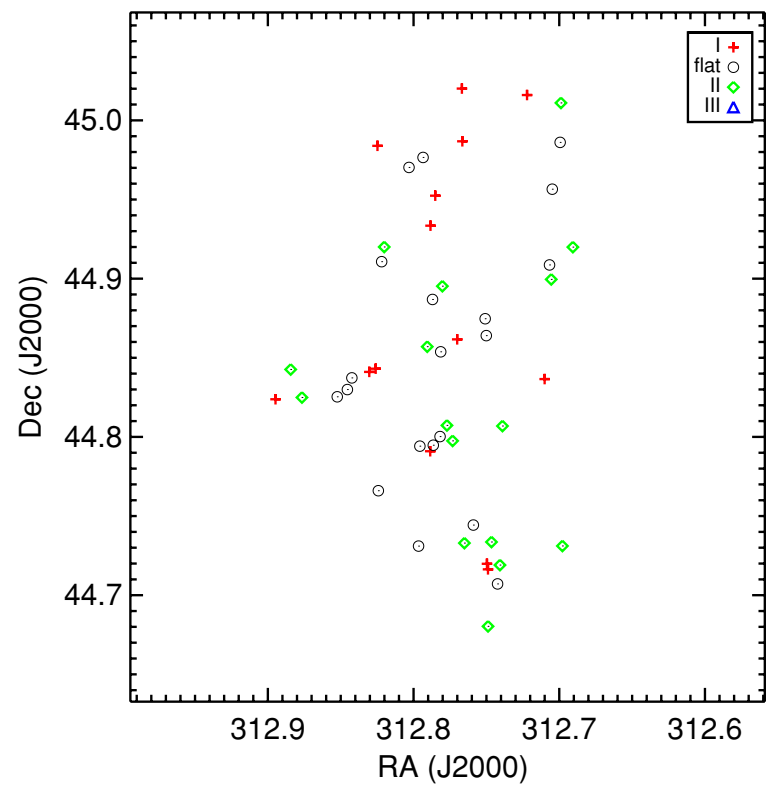

Figure 26. Location of YSOs, color-coded by their SED slope, in the Pelican's Hat cluster. Notation is as in similar earlier figures.

(A color version of this figure is available in the online journal.)

suggest youth; see Figure 25. About $8^{\prime}$ to the northeast of the north end of the dark lane (at 20:51:37, $+45: 05: 15$ or 312.9042, $+45.0875)$, there is a resolved patch of $24 \mu \mathrm{m}$ nebulosity, and $\sim 5.5$ to the west of the north end of the dark lane (at 20:50:35 $+44: 57: 16$, or 312.6458, +44.95444$)$, there is a resolved patch of nebulosity that might be a bipolar nebula, and it is on the edge of a larger circular feature $\sim 5.5$ in diameter. Since so many of the objects in the NAN are young, we hesitate to add these objects (and their apparently associated point sources) to the cluster definition, but they are likely YSOs, possibly from the same local episode of star formation that prompted the formation of objects in the Pelican's Hat cluster.

\section{IONIZING SOURCE(S)}

Many previous authors have sought the ionizing source, or sources, for the NAN; several articles have proposed various objects. Here we summarize that literature and then consider the Spitzer properties of several candidates.

Herbig (1958) considers and rejects the following stars as the exciting source of the nebula: 57 Cygni (too close, not enough reddening), $\mathrm{BD}+44^{\circ} 3627, \mathrm{BD}+42^{\circ} 3914$, and $\mathrm{MWC}$ 1032 (HD 198931) (all projected onto cloud but behind heavy obscuration), HD 199579 (orientation of bright rims not correct, O6 V not bright enough), $\alpha$ Cyg (Deneb; too far away), and IV Cygni association (orientation of bright rims not correct). He concludes, agreeing with Osterbrock (1957), that the source of excitation is likely behind the Gulf of Mexico.

The quest for the ionizing source of the complex continued in Neckel et al. (1980). They dismiss the O6 star HD 199579 as having two or three times too faint flux to account for the observed emission. They suggest an object that now can be identified as 2MASS J20535282+4424015. Their argument for this object being the exciting source includes the fact that it is bright in the IR, that it is $18^{\prime}$ from the radio geometric center of the nebula, and $8^{\prime}$ in front of the strongest radio-bright rim. They conclude that if it is the exciting source, it must have a type earlier than B1.

Wendker et al. (1983) discuss the fact that HD 199579 must be a background object; they assert that to match their long- 


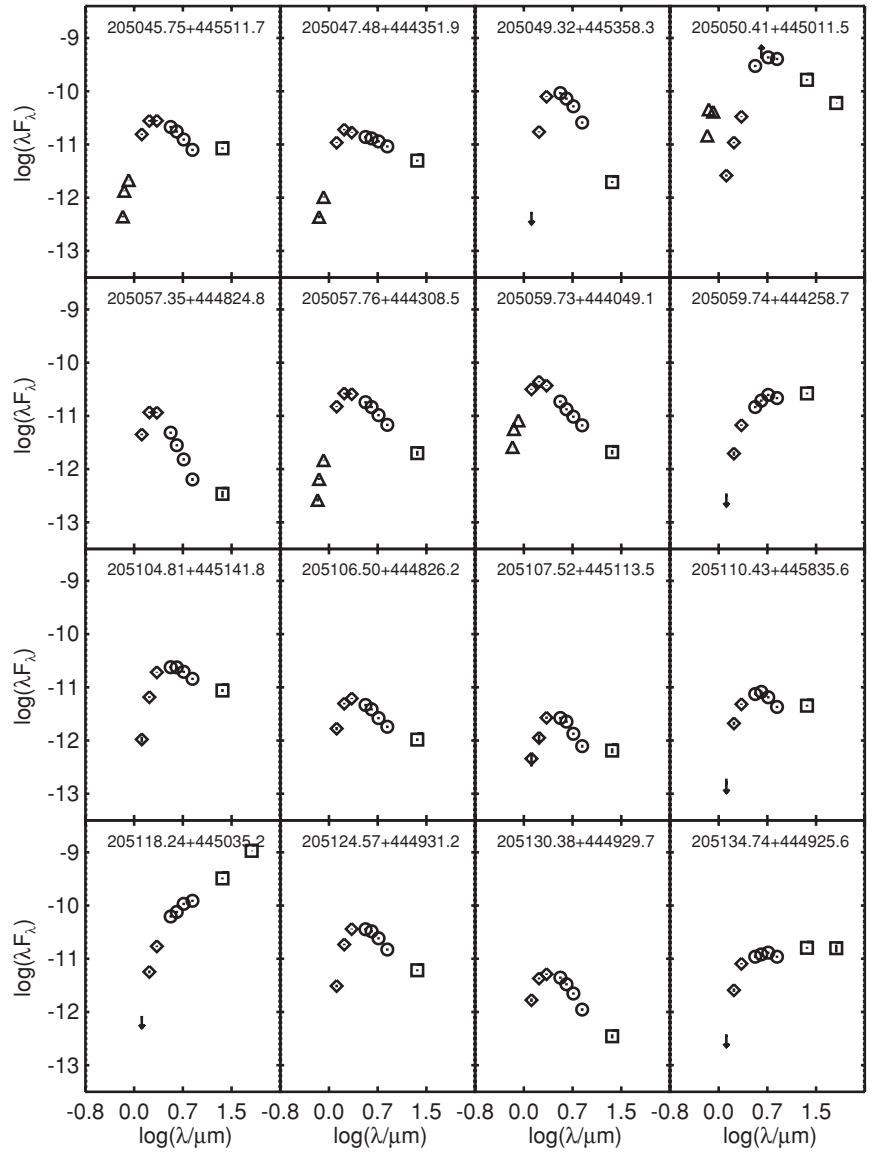

Figure 27. Sample of selected YSO candidate SEDs seen in the Pelican's Hat cluster; $\lambda F_{\lambda}$ is in erg s${ }^{-1} \mathrm{~cm}^{-2}$, and $\lambda$ is in $\mu \mathrm{m}$. All of these new objects have IAU-compatible names. Vertical error bars are typically smaller than the points. Symbols denote origin of photometry: $\times$ : Vilnius system, triangles: IPHAS, + Johnson (from the literature), diamonds: 2MASS, circles: IRAC, and squares: MIPS; downward-pointing arrows are upper limits.

wavelength observations, there must be a group of (as of then unknown) eight $\mathrm{O}$ stars powering the nebula.

Comerón \& Pasquali (2005) examined 19 bright preliminary candidates and concluded that 2MASS J205551.25+435224.6, an $\mathrm{O} 5 \mathrm{~V}$ (or earlier), is likely to be the ionizing source of the nebula. This object is also No. 10 from Bally \& Scoville (1980) and is located near the geometric center of the NAN based on radio observations. However, they were working on the assumption that the complex was at $\sim 610 \mathrm{pc}$, and they conclude based on that distance that an O5 star could be responsible for the observed bulk properties of the NAN. They observe that there are few other comparably early candidates in the immediate vicinity of this object, making this region distinctly different than regions such as the ONC where a cluster of one late $\mathrm{O}$ star and several early B stars powers the nebula.

Most recently, Straižys \& Laugalys (2008b) continue the search, finding 13 stars resembling heavily reddened $\mathrm{O}$ stars in this region, including the Comerón \& Pasqali (2005) source 2MASS J205551.25+435224.6. They confirm that it is an O5 $\mathrm{V}$, and a reasonable candidate for the exciting source of the nebula. Of the remaining 12 candidates, four of them could be $\mathrm{O}$ stars, contributing to the ionizing flux in the region. The rest are dismissed as background oxygen and carbon-rich AGB stars.

We can consider the SEDs of these objects based on the data we have amassed. In our multi-wavelength database, we do not have optical data for all of these objects, but for those that we do have, they are highly reddened. Most are very bright at 2MASS and Spitzer bands, and it could be that in reality all of the sources are highly reddened, but the optical data are saturated in the available surveys so are missing from our database. Several have at least one of their Spitzer flux densities compromised due to saturation (or near-saturation). For the ones where enough of the Spitzer measurements are viable, only one has any excess, MWC 1032 (HD 198931), and it is identified as a YSO candidate in the lists above. Two of the objects from Straižys \& Laugalys (2008b; their numbers 1 and 9) may have low-significance excesses; they were dropped from our list of YSO candidates during the vetting process above.

While it is unsurprising that the SEDs of many if not all of these candidate ionizing sources are highly reddened, it is interesting that one or possibly three have an infrared excess. If the stars are indeed young $\mathrm{O}$ or early $\mathrm{B}$ stars, it seems likely that they have already ablated or otherwise dispersed their circumstellar material, so no excess might be apparent. If the stars are later type B stars, disks could still be present.

Looking at the larger scales depicted in Figure 2, the limbbrightened edges of the MIPS-24 nebulosity appear to be roughly symmetric about the Gulf of Mexico, suggesting that perhaps the illuminating source really is behind the Gulf, and thus may be a challenge to recover. The Comerón \& Pasquali star (2MASS J205551.25+435224.6) is located north of the Gulf of Mexico, very roughly at the "Bahamas," which is roughly consistent with this illumination pattern.

\section{CONCLUSIONS}

We have presented Spitzer/MIPS observations of $\sim 7 \mathrm{deg}^{-2}$ of the North American Nebula molecular cloud complex at 24, 70, and $160 \mu \mathrm{m}$. There is large-scale and complex structure in the ISM observed in all bands. The $160 \mu \mathrm{m}$ emission follows the near-IR-derived $A_{V}$ contours. About 4300 point sources are seen at $24 \mu \mathrm{m}$, and $\sim 100$ point sources are seen at $70 \mu \mathrm{m}$. We merged this catalog to that derived from G09 for the Spitzer/IRAC data, as well as extensive optical and near-IR data.

We have assembled a catalog of the previously known objects in this region, focusing on the $\sim 200$ previously identified YSOs and candidates. (As supplemental material, we provide a list of these objects not recovered by our search.) We matched this catalog to that derived from our Spitzer MIPS and IRAC data. We used a series of color criteria (plus other criteria, such as distance from the center of the complex) to select objects as YSO candidates. Using our MIPS-focused criteria, we identified 1286 candidates, most of which were independently identified using IRAC-focused criteria. Some of the IRAC-selected YSO candidates were not recovered using the MIPS criteria because they were not detected at the less sensitive MIPS bands. When the total number of unique YSO candidates as identified from MIPS and IRAC are combined, we have identified 2076 YSO candidates, about 10 times as many YSOs as were previously known here. Most of our YSO candidates are Class II YSOs.

Since the North American Nebula/Pelican Nebula region is in the Galactic plane and is seen along a spiral arm, contamination rates could be high. Our most likely lingering contaminant is AGB stars; follow-up spectroscopy is needed to confirm the candidates as YSOs, or identify them as contaminants. Additional observations such as X-ray data and variability (monitoring) campaigns will also help in eliminating contaminants.

A clustering analysis of the distribution of YSO candidates suggests at least three clusters, named for their location in the complex: the Gulf of Mexico, the Pelican, and the Pelican's Hat. 
The Gulf of Mexico cluster includes 283 MIPS-selected YSO candidates, and surrounds a dramatic sinuous dust lane, which is dark even out to $70 \mu \mathrm{m}$. It is subject to the highest extinction $\left(A_{V}\right.$ at least $\sim 30$ ) and has the widest range of infrared colors of the three clusters, including the largest excesses and by far the most point-source detections at $70 \mu \mathrm{m}$. Just $3 \%$ of the cluster members were previously identified, and we have defined this cluster as about 10-100 times larger (in projected area) than was previously realized.

The Pelican cluster is comparable in number of objects to the Gulf of Mexico (247 MIPS-selected YSO candidates), but is subject to much less reddening, and has a much higher fraction of less-embedded objects (based on SED shape). It includes a relatively high fraction (20\%) of previously identified objects, but the cluster boundaries are much different than what was previously recognized.

The Pelican's Hat cluster is an entirely new discovery, with no prior identifications of YSOs. It surrounds a dark lane somewhat similar in morphology to (though much smaller than) the Gulf of Mexico. It contains only 51 MIPS-selected YSO candidates, though it is close to objects with interesting morphology in the $24 \mu \mathrm{m}$ image, bearing further study.

We consider various ionizing source candidates and conclude that some of the candidates under consideration may have IR excesses. The illumination pattern is consistent with the ionizing star suggested by Comerón \& Pasquali (2005), but it is also possible that some ionizing source(s) are behind the Gulf of Mexico.

As additional material, we compared our source lists and images to those available from IRAS, MSX, and AKARI, finding that the Spitzer data resolve ISM and source confusion. We also discuss some candidate clusters within and projected onto the NAN which are otherwise unremarkable in our data. Some objects from the superimposed cluster NGC 6997 may be debris disks.

The authors wish to thank the following people: V. Straižys for a helpful article referee report; the c2d team for use of their SWIRE reduction; V. Straižys and collaborators for providing machine-readable tables (and coordinate checks) for several of their publications prior to submission.

This research has made use of NASA's Astrophysics Data System (ADS) Abstract Service, and of the SIMBAD database, operated at CDS, Strasbourg, France. This research has made use of data products from the Two Micron All-Sky Survey (2MASS), which is a joint project of the University of Massachusetts and the Infrared Processing and Analysis Center, funded by the National Aeronautics and Space Administration and the National Science Foundation. These data were served by the NASA/IPAC Infrared Science Archive, which is operated by the Jet Propulsion Laboratory, California Institute of Technology, under contract with the National Aeronautics and Space Administration. This research has made use of the Digitized Sky Surveys, which were produced at the Space Telescope Science Institute under U.S. Government grant NAG W-2166. The images of these surveys are based on photographic data obtained using the Oschin Schmidt Telescope on Palomar Mountain and the UK Schmidt Telescope. The plates were processed into the present compressed digital form with the permission of these institutions. This paper makes use of data obtained as part of the INT Photometric H $\alpha$ Survey of the Northern Galactic Plane (IPHAS) carried out at the Isaac Newton Telescope (INT). The INT is operated on the island of La Palma by the Isaac Newton
Group in the Spanish Observatorio del Roque de los Muchachos of the Instituto de Astrofisica de Canarias. All IPHAS data, processed by the Cambridge Astronomical Survey Unit, were obtained via the database and image access provided by Astrogrid.

The research described in this paper was partially carried out at the Jet Propulsion Laboratory, California Institute of Technology, under contract with the National Aeronautics and Space Administration.

\section{APPENDIX A}

\section{COMPARISON TO IRAS, MSX, AND AKARI}

Because Spitzer goes deeper and has higher resolution than prior infrared missions such as IRAS and $M S X$, and the current mission $A K A R I$, by comparing the data, we can learn how well these other missions did in identifying point sources in a region such as this with complex nebulosity and high source densities, both leading to confusion.

\section{A.1. IRAS}

IRAS flew in 1983 and observed at 12, 25, 60, and $100 \mu \mathrm{m}$. The angular resolution of IRAS ranged from $\sim 0.5$ at $12 \mu \mathrm{m}$ to $\sim 2^{\prime}$ at $100 \mu \mathrm{m}$; source confusion is clearly a problem in regions like the NAN, where the source density varies from $\sim 20 \operatorname{arcmin}^{-2}$ at IRAC bands to $\sim 0.2 \operatorname{arcmin}^{-2}$ at $24 \mu \mathrm{m}$, and the nebular emission is complex at essentially all bands.

Previous results in Perseus from Rebull et al. (2007) comparing MIPS and IRAS data suggest that the IRAS point-source catalog (PSC; Beichman et al. 1988) and faint-source catalog (FSC; Moshir et al. 1992) did reasonably well in identifying true point sources; while the extended emission is complicated in Perseus (as it is in the NAN), the source density is not as high as in the NAN.

IRAS surveyed more than $96 \%$ of the sky, but one of those missing sky segments is in the NAN region. There is a missing "wedge" of IRAS imaging data along the eastern edge of our map. Apparently as a result, the point-source extraction routines were not run over much of the NAN map. There are no FSC sources found at all within $4 \mathrm{deg}^{2}$ radius of our map center. There are only PSC sources found in a narrow strip in the western edge of our map, from ecliptic longitudes $\sim 336^{\circ}$ to $337^{\circ}$.

There are 69 PSC objects that overlap our MIPS map, 28 of which are listed as solid detections (quality flag $=3$ ) at $25 \mu \mathrm{m}$, and they appear in Table 7. Thirteen of these resolve into nebulosity when viewed at $24 \mu \mathrm{m}$; the rest resolve into at least one point source. At $60 \mu \mathrm{m}$, there are 28 solid detections (quality flag $=3$ ), just three of which are detected as point sources in our $70 \mu \mathrm{m}$ map (IRAS 20472+4322, 20467+4340, and $20472+4338$ ); the rest are confused by nebulosity. The nebulosity is complex, and the overall sky brightness is high, in this region.

While MIPS has much more sensitive detectors than IRAS, it also samples a much smaller beamsize, so the achieved surface brightness sensitivity is not tremendously improved over IRAS. Most of the large-scale structure seen in the MIPS maps can also be seen in the IRAS maps. Obviously the tremendous texture found in the MIPS maps is lost in the IRAS maps.

\section{A.2. $M S X$}

The NAN region is included in the $M S X$ survey of the Galactic plane (Price et al. 2001). A U.S. Air Force satellite, MSX flew in 
Table 7

Comparison to IRAS $25 \mu \mathrm{m}$

\begin{tabular}{|c|c|}
\hline IRAS $25 \mu \mathrm{m}$ Source & Notes \\
\hline $20443+4441$ & Point source at $24 \mu \mathrm{m}$ \\
\hline $20445+4436$ & Point source at $24 \mu \mathrm{m}$ \\
\hline $20447+4441$ & Point source at $24 \mu \mathrm{m}$ (cluster?) \\
\hline $20453+4441$ & Point source at $24 \mu \mathrm{m}$ \\
\hline $20458+4416$ & Point source at $24 \mu \mathrm{m}$ \\
\hline $20467+4340$ & Point source at $24 \mu \mathrm{m}$ \\
\hline $20468+4410$ & Nebulosity \\
\hline $20469+4352$ & Nebulosity \\
\hline $20469+4435$ & Point source at $24 \mu \mathrm{m}$ \\
\hline $20472+4338$ & Point source at $24 \mu \mathrm{m}$ \\
\hline $20472+4411$ & Nebulosity \\
\hline $20474+4416$ & Nebulous knot \\
\hline $20475+4431$ & Nebulosity/maybe point source at $24 \mu \mathrm{m}$ \\
\hline $20476+4422$ & Point source at $24 \mu \mathrm{m}$ \\
\hline $20479+4419$ & Nebulosity \\
\hline $20479+4438$ & Nebulosity \\
\hline $20481+4404$ & Point source at $24 \mu \mathrm{m}$ \\
\hline $20485+4407$ & Nebulosity \\
\hline $20485+4423$ & Nebulosity \\
\hline $20489+4410$ & Nebulosity/cluster of point sources at $24 \mu \mathrm{m}$ ? \\
\hline $20490+4351$ & Point source at $24 \mu \mathrm{m}$ \\
\hline $20490+4417$ & Bright nebulosity \\
\hline $20495+4320$ & Nebulosity \\
\hline $20496+4354$ & Point source at $24 \mu \mathrm{m}$ \\
\hline $20499+4337$ & Two point sources at $24 \mu \mathrm{m}$ \\
\hline $20511+4225$ & Nebulous point source at $24 \mu \mathrm{m}$ \\
\hline $20516+4304$ & Point source at $24 \mu \mathrm{m}$ \\
\hline $20524+4227$ & point source at $24 \mu \mathrm{m}$ \\
\hline
\end{tabular}

1996-1997, and observed at $8.28,12.13,14.65$, and $21.3 \mu \mathrm{m}$ at a resolution of $\sim 18^{\prime \prime}$. As for the IRAS maps, hints of the structure found in the ISM can be seen in the lower-spatial-resolution MSX images. The dark lane in the Gulf of Mexico can clearly be seen in the $8.28 \mu \mathrm{m}$ image (and hints of it can even be seen the $12.13 \mu \mathrm{m}$ image). The brightest emission regions discussed in Section 2.3 can be seen in the MSX images as well: the Pelican's neck, the Mexican Riviera, and the bright resolved blobs.

About 900 sources are retrieved from the MSX point-source catalog (Egan et al. 1999) for this region. Simply by overlaying them on the Spitzer images, it can be seen that many are clearly confused by the ridges and structure in the nebulosity, as well as point-source confusion itself, though many do correspond to real point sources seen in the $24 \mu \mathrm{m}$ data. It is somewhat difficult to compare the flux densities quantitatively, as the Spitzer instruments saturate at about the point where the MSX channels are operating at the lower limit of their completeness. There are $\sim 20$ sources in common between $M S X$ Band A $(8.28 \mu \mathrm{m})$ and IRAC $8 \mu \mathrm{m}$, most of which are reasonably well matched in measured flux densities ( $1 \sigma$ scatter for the brighter sources is $17 \%$ ); about $20 \%$ of the IRAC observations clearly resolve source confusion in the MSX data. There are $\sim 40$ sources in common between MSX Band E $(21.34 \mu \mathrm{m})$ and MIPS $24 \mu \mathrm{m}$, the brighter half of which are comparable in measured flux densities ( $1 \sigma$ scatter for the brighter sources is $37 \%$ ); about $40 \%$ clearly resolve source confusion in the $M S X$ data, most of which are below the $2 \mathrm{Jy}$ completeness limit for the MSX Band E data.

\section{A.3. AKARI}

AKARI is a Japanese mission, and it surveyed the whole sky at $9,18,65,90,140$, and $160 \mu \mathrm{m}$ during its cryogenic phase (2006-2007). With a primary mirror of $68.5 \mathrm{~cm}, A K A R I$ 's resolution is slightly worse than Spitzer's; the spatial resolution is $\sim 5^{\prime \prime}$ at the shortest bands and $\sim 90^{\prime \prime}$ at the longest. As of this writing, images from this survey are not available, but catalogs are. We extracted $\sim 1100$ sources in this region from the IRC 9 and $18 \mu \mathrm{m}$ all-sky point-source catalog (Ishihara et al. 2010) and $\sim 2100$ sources in this region from the FIS 65, 90, 150, and $160 \mu \mathrm{m}$ all-sky bright source catalog (Yamamura et al. 2009). Not all of those sources are indicated to be high-quality detections at each band; $96 \%$ of the IRC catalog is a highquality detection at $9 \mu \mathrm{m}$, whereas just $30 \%$ are similar quality detections at $18 \mu \mathrm{m}$. For the FIS catalog, 13\%, 92\%, 31\%, and $15 \%$ are high-quality detections at $65,90,140$, and $160 \mu \mathrm{m}$, respectively.

By empirical inspection of the Spitzer images, the IRC catalog is not often confused by nebulosity; the overwhelming majority of IRC point sources are point sources for Spitzer as well. Unsurprisingly, the Spitzer data are substantially deeper; the AKARI catalogs only go to $\sim 0.1 \mathrm{Jy}$ at $9 \mu \mathrm{m}$. There are $\sim 640$ objects in common between the catalogs, and the flux densities are reasonably well matched between the AKARI $9 \mu \mathrm{m}$ and the Spitzer $8 \mu \mathrm{m}$ channels ( $1 \sigma$ scatter is $27 \%$ ), keeping in mind not only filter variation but intrinsic stellar variability.

In contrast, the FIS catalog seems more often to not recover point sources seen in the Spitzer images. Without the AKARI images to compare, it is difficult to understand many of the FIS catalog detections in this confused and complex region. Only about $20(<1 \%)$ of the FIS catalog detections correspond by eye to $70 \mu \mathrm{m}$ Spitzer point or point-like sources. A significant fraction of the sources are clearly confused by structure in the nebulosity (e.g., there are "chains" of sources along emission ridges).

\section{APPENDIX B}

\section{CLUSTERS NOT PART OF THE NAN}

There are some clusters identified in the literature that are likely foreground objects, or are not recovered in the Spitzer data. We discuss these here.

\section{B.1. NGC 6997}

NGC 6997 has been identified historically as being a cluster superimposed either in front of or behind the NAN complex. Note that this cluster has been commonly confused with NGC 6996; see Laugalys et al. (2006b) and Corwin (2004). NGC 6997 has been recently studied by three papers in the literature. Zdanavičius \& Straižys (1990) identified 15 cluster members, placing the distance at $620 \mathrm{pc}$ (whereas the NAN is taken in that work to be at $550 \mathrm{pc}$ ) and the age at $\sim 10^{8}$ years. Villanova et al. (2004) double the cluster spatial size, finding $\sim 50$ new probable or likely members, and placing the cluster at $\sim 760 \mathrm{pc}$ and $\sim 350$ Myr, in front of their distance for the NAN complex (which they take to be $1 \mathrm{kpc}$ ). Laugalys et al. (2006b) find $\sim 50$ more members, place the cluster at 780-790 $\mathrm{pc}$ (now again behind the NAN), and estimate the age at $3 \times 10^{8}$ years. It is identified in Figure 16 above.

For our discussion here, we do not attempt to redefine the cluster boundaries; we simply accept as legitimate members the 112 objects identified in these papers (and detected in our Spitzer survey) as cluster members, and it does not matter if the cluster is in front of or behind the NAN. On the whole, the stellar population of NGC 6997 indeed looks different than the rest of the cloud. None of the stars currently identified as 


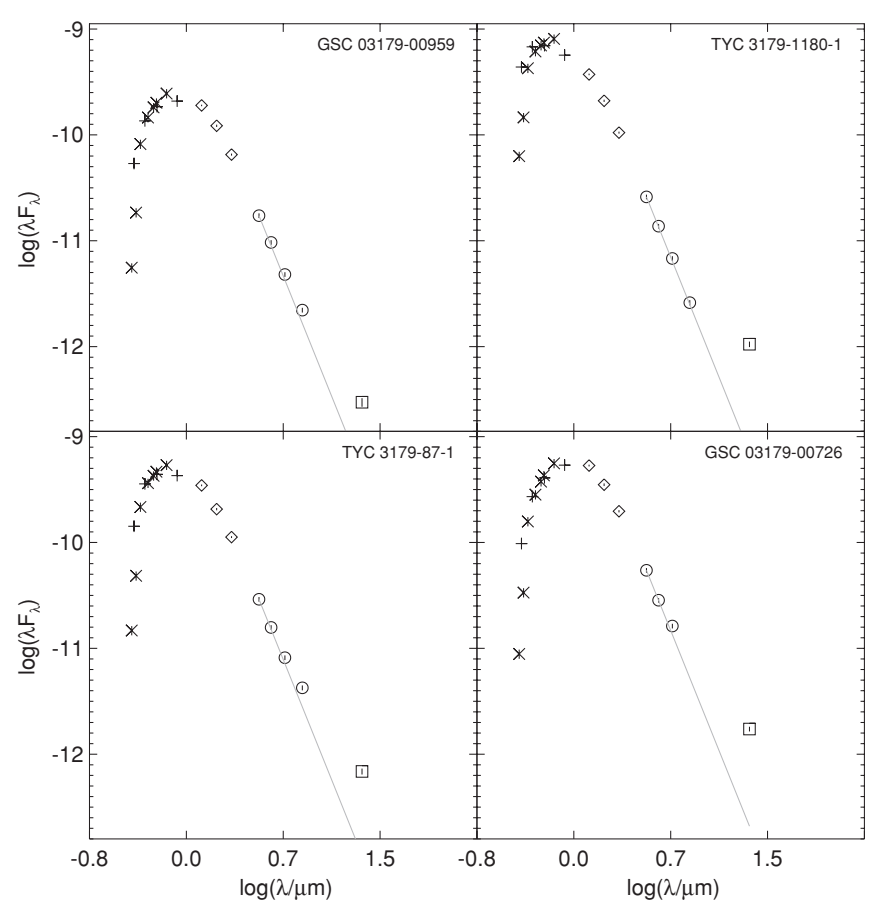

Figure 28. SEDs for the four high-confidence YSO candidates which have also been identified as NGC 6997 members; $\lambda F_{\lambda}$ is in erg s${ }^{-1} \mathrm{~cm}^{-2}$, and $\lambda$ is in $\mu \mathrm{m}$. Notation is as in prior SED figures; the gray line from $3.6 \mu \mathrm{m}$ traces the flux expected from a photosphere. If these objects are really NGC 6997 members, and if the cluster is $\sim 350 \mathrm{Myr}$ old, then these are candidate debris disks, though the apparent IR excess could be due to source confusion (including with a low-mass companion).

NGC 6997 members have IRAC excesses, consistent with the cluster being older than (and probably unrelated to) the NAN complex. Twelve of the 112 objects are seen at $24 \mu \mathrm{m}$, and none at $70 \mu \mathrm{m}$. Out of these 12 objects, four are identified as high-confidence YSO candidates. The SEDs for the four high-confidence YSO candidates appear in Figure 28; they are GSC 03179-00959 (205622.0+443537.2), TYC 3179-1180-1 (205624.6+443921.3), TYC 3179-87-1 (205633.1+443810.3), and GSC 03179-00726 (205639.5+443821.6). There are two more lower-confidence YSO candidates (GSC 03179$01093=205633.3+443622.3$ and GSC 03179-00980 = 205637.1+444009.2). These latter two objects have much lowersignificance excesses at $24 \mu \mathrm{m}$. All of these YSO candidates appear in the complete tables of YSO candidates above.

If these stars are truly members of NGC 6997, then these observed excesses could be only apparent excesses, due to source confusion or an unresolved lower-mass companion, or they could be real excesses. If they are real, then they are likely debris disks. The presence of such debris disks is broadly consistent with the age of the cluster being $\sim 350$ Myr. Cieza et al. (2008) find, for the Hyades ( $650 \mathrm{Myr})$, that none of the FGK stars have debris disks, but that 2 of 11 A stars have debris disks. For these four high-confidence YSO candidates in NGC 6997, the spectral types from the literature are G7 V, B5 V, A3 V, and A7 III:, respectively; again, this is broadly consistent with the literature. A more complete study of the membership of this cluster would allow a more complete estimate of the disk fraction of this cluster.

We conclude that this cluster (as defined in the literature) is sufficiently different in mid-infrared properties from the NAN that it is unlikely to be related to the NAN, or at least much older than the rest of the complex.

\section{B.2. Collinder 428}

Collinder 428 has been identified historically as being a cluster on the far east side of the complex that is likely unrelated to the NAN complex. Laugalys et al. (2007) concluded that the dust cloud in the direction of Collinder 428 is at the same distance as the NAN complex and that the star grouping is not a real cluster but rather a relatively transparent window in the dust cloud, and therefore not a separate object.

The stars in this region are on the edge of our map, and none of them are identified as YSO candidates using either IRAC or MIPS. Such a low disk fraction implies that if Collinder 428 is a real cluster, it is not part of the NAN complex. This is consistent with the Laugalys et al. (2007) interpretation that the stellar grouping is illusory due to a window in the dust cloud and not a separate entity.

\section{B.3. Remaining Cambrésy et al. (2002) Clusters}

Cambrésy et al. (2002) identified eight clusters based on 2MASS data. Clusters 1, 2, and $3 \mathrm{a} / \mathrm{b}$ are part of the Gulf of Mexico, and cluster 5 is part of the Pelican cluster, as described above. We now discuss the remaining clusters. We do not define for further consideration any additional clusters associated with any of these objects, for reasons that will become apparent.

Cluster 4 appears in the $24 \mu \mathrm{m}$ image as wispy faint $(\sim 40$ MJy sr ${ }^{-1}$ ) nebulosity, with the nearest very faint point source $2^{\prime}$ away $(0.3 \mathrm{pc}$ at $520 \mathrm{pc})$, and the nearest point source of a brightness range comparable to the clusters above nearly $\sim 7.5$ away $(1.3 \mathrm{pc})$.

Cluster 6 appears in the $24 \mu \mathrm{m}$ image as the heart of a roughly arc-shaped patch of bright $\left(\lesssim 650 \mathrm{MJy} \mathrm{sr}^{-1}\right)$ feathery nebulosity roughly $11^{\prime} \times 3^{\prime}(1.7 \times 0.5 \mathrm{pc}$ at $520 \mathrm{pc})$. No point sources can be discerned on top of the nebulosity, though some faint point sources are seen close to the nebulosity.

Cluster 7 appears in the $24 \mu \mathrm{m}$ image as a circular patch of bright (250-650 $\left.\mathrm{MJy} \mathrm{sr}^{-1}\right)$ nebulosity $\sim 4^{\prime}(0.6 \mathrm{pc}$ at $520 \mathrm{pc})$ in diameter. No point sources can be detected on top of this nebulosity.

Cluster 8, seen in 2MASS images as a tight, red clumping, appears as a single very bright point source at $24 \mu \mathrm{m}$, with no particular higher density of surrounding point sources at $24 \mu \mathrm{m}$.

\section{APPENDIX C}

\section{YSO CANDIDATES NOT RECOVERED BY OUR MIPS-BASED SEARCH}

There are two categories of objects that our primarily MIPSbased search does not recover: objects seen to have an IRAC excess but not detected at MIPS bands (likely to be primarily the lower mass members of the cluster), and objects previously identified as YSO candidates that do not have IR excesses (these were likely to have been identified as YSOs using other methods than the search for IR excesses). Here, for completeness, we present flux density tables for these objects.

\section{C.1. IRAC-only YSOs}

In Table 8, we list the Spitzer fluxes (and cross-identifications) for those objects identified using the G09 methodology but not rediscovered using the MIPS-based selection mechanisms here.

\section{C.2. Unrecovered YSO Candidates}

In Table 9, we list the Spitzer fluxes (and cross-identifications) for those objects identified by the literature as YSO candidates 
IRAC-selected YSOs Not Recovered by Our MIPS-based Search

\begin{tabular}{|c|c|c|c|c|c|c|c|c|c|c|c|c|}
\hline Name & R.A. (J2000) & Decl. (J2000) & 2MASS Name & Alt. Name & Sp. Ty. & Sp. Ty. Src & [3.6] (mag) & [4.5] (mag) & [5.8] (mag) & [8.0] (mag) & [24] (mag) & [70] (mag) \\
\hline $204717.51+434749.8$ & 311.822968 & 43.797180 & $20471748+4347498$ & & & & $12.37 \pm 0.05$ & $11.99 \pm 0.06$ & $11.64 \pm 0.06$ & $11.10 \pm 0.06$ & $\ldots$ & \\
\hline $204722.36+435011.0$ & 311.843170 & 43.836414 & $20472236+4350114$ & .. & $\ldots$ & $\ldots$ & $11.86 \pm 0.05$ & $11.65 \pm 0.05$ & $11.47 \pm 0.06$ & $10.71 \pm 0.06$ & 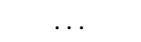 & . \\
\hline $204723.59+434439.5$ & 311.848297 & 43.744324 & $20472359+4344397$ & LkHa 371 & A3 & Terranegra94 & $7.61 \pm 0.05$ & $7.17 \pm 0.05$ & $6.62 \pm 0.05$ & $5.46 \pm 0.05$ & $\ldots$ & \\
\hline $204725.20+434832.0$ & 311.855011 & 43.808903 & $20472521+4348322$ & & $\cdots$ & & $10.01 \pm 0.05$ & $9.47 \pm 0.05$ & $9.03 \pm 0.05$ & $8.13 \pm 0.05$ & .. & . \\
\hline $204726.00+434908.9$ & 311.858368 & 43.819164 & $20472600+4349093$ & . & $\cdots$ & $\ldots$ & $14.13 \pm 0.06$ & $13.84 \pm 0.06$ & $13.63 \pm 0.10$ & $12.98 \pm 0.11$ & ... & $\cdots$ \\
\hline $204727.03+434912.7$ & 311.862640 & 43.820198 & $20472705+4349129$ & $\cdots$ & $\ldots$ & .. & $13.78 \pm 0.06$ & $13.57 \pm 0.06$ & $13.34 \pm 0.09$ & $13.01 \pm 0.14$ & . & $\cdots$ \\
\hline $204727.89+435022.7$ & 311.866211 & 43.839642 & $20472789+4350227$ & $\cdots$ & $\ldots$ & $\cdots$ & $13.61 \pm 0.06$ & $13.38 \pm 0.06$ & $13.04 \pm 0.07$ & $12.62 \pm 0.08$ & .. & $\cdots$ \\
\hline $204730.40+434612.1$ & 311.876679 & 43.770054 & $20473041+4346121$ & .. & $\ldots$ & .. & $11.10 \pm 0.05$ & $10.43 \pm 0.05$ & $9.94 \pm 0.06$ & $9.46 \pm 0.06$ & $\cdots$ & . \\
\hline $204734.18+434723.9$ & 311.892456 & 43.789978 & $20473417+4347239$ & $\ldots$ & $\ldots$ & $\ldots$ & $11.53 \pm 0.06$ & $11.14 \pm 0.06$ & $10.50 \pm 0.06$ & $9.74 \pm 0.06$ & $\ldots$ & $\ldots$ \\
\hline $204735.33+434446.0$ & 311.897247 & 43.746121 & $20473531+4344459$ & $\ldots$ & $\ldots$ & $\ldots$ & $13.83 \pm 0.06$ & $13.56 \pm 0.06$ & $13.25 \pm 0.08$ & $12.47 \pm 0.09$ & $\cdots$ & $\ldots$ \\
\hline
\end{tabular}

(This table is available in its entirety in a machine-readable form in the online journal. A portion is shown here for guidance regarding its form and content.)

Table 9

Previously Suggested YSOs Not Recovered by Our MIPS or IRAC Searches

\begin{tabular}{|c|c|c|c|c|c|c|c|c|c|c|c|c|}
\hline Name & R.A. (J2000) & Decl. (J2000) & 2MASS Name & Alt. Name & Sp. Ty. & Sp. Ty. Src & [3.6] (mag) & [4.5] (mag) & [5.8] (mag) & [8.0] (mag) & [24] (mag) & [70] (mag) \\
\hline $204624.97+445545.8$ & 311.604070 & 44.929396 & $20462500+4455449$ & GSC 03178-00815 & $\mathrm{Be}$ & MerrillBurwell49 & & & & $\cdots$ & $4.84 \pm 0.04$ & $\cdots$ \\
\hline $204636.80+434434.9$ & 311.653351 & 43.743038 & $20463682+4344353$ & LkHa 131 & O8e & Terranegra94 & $9.67 \pm 0.05$ & $\ldots$ & $9.15 \pm 0.05$ & $\ldots$ & $\ldots$ & . \\
\hline $204641.54+434648.5$ & 311.673096 & 43.780155 & $20464155+4346488$ & LkHa 132 & B4 & CohenKuhi79 & $11.64 \pm 0.05$ & & $11.31 \pm 0.06$ & & $\ldots$ & \\
\hline $204645.64+434511.4$ & 311.690185 & 43.753174 & $20464565+4345114$ & AS 441 & B7.5 & Terranegra94 & $8.15 \pm 0.05$ & & $6.53 \pm 0.05$ & & & \\
\hline $204728.16+445711.4$ & 311.867361 & 44.953191 & $20472816+4457114$ & GSC 03178-00107 & G-K & Welin73 & & & & & $7.32 \pm 0.05$ & \\
\hline $204737.47+434724.8$ & 311.906128 & 43.790226 & $20473745+4347249$ & AS 442 & B8.5 & Terranegra94 & $<6.62$ & $<6.10$ & $3.97 \pm 0.05$ & $<3.52$ &. & \\
\hline $204803.23+444541.6$ & 312.013489 & 44.761562 & $20480324+4445417$ & BD+44 3587 & B7 & Welin73 & $8.74 \pm 0.05$ & 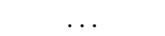 & $8.70 \pm 0.05$ & & $8.74 \pm 0.07$ & \\
\hline $204804.78+434725.5$ & 312.019928 & 43.790432 & $20480478+4347258$ & LkHa 134 & B7 & Terranegra94 & $<6.62$ & $6.16 \pm 0.05$ & $5.55 \pm 0.05$ & $3.80 \pm 0.05$ & $\ldots$ & \\
\hline $204911.58+452440.1$ & 312.298279 & 45.411140 & $20491158+4524398$ & $\mathrm{BD}+44 \mathrm{~d} 3594$ & $\mathrm{~B} 0 \mathrm{e}$ & MerrillBurwell49 & $\ldots$ & $7.11 \pm 0.05$ & & $6.48 \pm 0.05$ & $\cdots$ & $\cdots$ \\
\hline $204933.77+450336.4$ & 312.390747 & 45.060131 & $20493376+4503364$ & GSC 03575-06527 & $\mathrm{F} 4$ & Welin73 & $9.82 \pm 0.05$ & $9.84 \pm 0.05$ & $9.81 \pm 0.05$ & $9.83 \pm 0.05$ & $\ldots$ & $\ldots$ \\
\hline
\end{tabular}

(This table is available in its entirety in a machine-readable form in the online journal. A portion is shown here for guidance regarding its form and content.) 
but not rediscovered using either the MIPS-based selection mechanism that was the primary goal of this paper, or the IRAC-based selection mechanism from G09 and reported in the prior table. These objects may or may not be legitimate NAN members; we cannot determine this from our data set.

Note that six objects were identified in Witham et al. (2008) as bright in $\mathrm{H} \alpha$ in this region, but were not identified by us here as having an IR excess. All six of these objects are undetected at MIPS bands and on the edges of the IRAC maps such that they are only detected at two of the four IRAC bands. As such, they would not have been identified by our YSO identification approaches. They are objects 204654.78+434806.6, 204703.24+434930.0, 204704.82+ $434911.2,205934.89+452355.9,205938.69+452414.5$, and $210029.50+443655.4$.

\section{REFERENCES}

Allen, L. E., et al. 2004, ApJS, 154, 363

Bally, J., \& Reipurth, B. 2003, AJ, 126, 893

Bally, J., \& Scoville, N. 1980, ApJ, 239, 121

Beichman, C. A., Neugebauer, G., Habing, H., Clegg, P., \& Chester, T. 1988, IRAS Catalog and Explanatory Supplement (Pasadena, CA: IPAC)

Billot, N., Noriega-Crespo, A., Carey, S., Guieu, S., Shenoy, S., Paladini, R., \& Latter, W. 2010, ApJ, 712, 797

Blum, R., et al. 2006, AJ, 132, 2034

Briggs, K., et al. 2007, A\&A, 468, 413

Cambrésy, L., Beichman, C., Jarrett, T., \& Cutri, R. 2002, AJ, 123, 2559

Carey, S., et al. 2009, PASP, 121, 76

Cieza, L., Swift, J., Mathews, G., \& Williams, J. 2008, ApJ, 679, 720

Clarke, C., \& Bouvier, J. 2000, MNRAS, 319, 457

Cohen, M., \& Kuhi, L. 1979, ApJS, 41, 743

Comerón, F., \& Pasquali, A. 2005, A\&A, 430, 541

Corbally, C., Straižys, V., \& Laugalys, V. 2009, Baltic Astron., 18, 111

Corwin, H. G. 2004, History and Accurate Positions for the NGC/IC Objects, CDS Catalog VII/239A, http://vizier.cfa.harvard.edu/viz-bin/VizieR?source $=\mathrm{VII} \% 2 \mathrm{~F} 239 \mathrm{~A}$

Covey, K., et al. 2011, AJ, 141, 40

Désert, F.-X., Boulanger, F., \& Puget, J. L. 1990, A\&A, 237, 215

Drew, J., et al. 2005, MNRAS, 362, 753

Egan, M. P., Price, S. D., Moshir, M., Cohen, M., \& Tedesco, E. 1999, The Midcourse Space Experiment Point Source Catalog Version 1.2 Explanatory Guide, Technical Report AFRL-VS-TR-1999-1522 (Hanscom AFB, MA: Air Force Res. Lab.)

Engelbracht, C., et al. 2007, PASP, 119, 994

Evans, N., et al. 2003, PASP, 115, 965

Evans, N., et al. 2009, ApJS, 181, 321

Fazio, G., et al. 2004, ApJS, 154, 10

Feldt, C. 1993, A\&A, 276, 531

Feldt, C., \& Wendker, H. 1993, A\&AS, 100, 287

Flagey, N., et al. 2009, ApJ, 701, 1450

Gautier, T. N., et al. 2007, ApJ, 667, 527

Gordon, K., et al. 2005, PASP, 117, 503

Guieu, S., et al. 2009, ApJ, 697, 787 (G09)

Gutermuth, R., Megeath, S. T., Myers, P. C., Allen, L. E., Pipher, J. L., \& Fazio, G. G. 2009, ApJS, 184, 18

Gutermuth, R., et al. 2008, ApJ, 674, 336

Harvey, P., Merín, B., Huard, T., Rebull, L., Chapman, N., Evans, N., \& Myers, P. 2007a, ApJ, 663, 1149

Harvey, P., et al. 2007b, ApJ, 663, 1139

Harvey, P., et al. 2006, ApJ, 644, 307

Herbig, G. 1958, ApJ, 128, 259

Ishihara, D., et al. 2010, A\&A, 514, A1

Kenyon, S., Gómez, M., \& Whitney, B. 2008, in Handbook of Star Forming Regions, Vol. I: The Northern Sky, ed. B. Reipurth (ASP Monograph Publications, Vol. 4; San Francisco, CA: ASP), 405
Kraus, A., \& Hillenbrand, L. 2007, ApJ, 662, 413

Laugalys, V., \& Straižys, V. 2002, Baltic Astron., 11, 205

Laugalys, V., Straižys, V., Vrba, F. J., Boyle, R. P., Davis Philip, A. G., \& Kazlauskas, A. 2006a, Baltic Astron., 15, 483

Laugalys, V., Straižys, V., Vrba, F. J., Boyle, R. P., \& Davis Philip, A. G. 2006b, Baltic Astron., 15, 327

Laugalys, V., Straižys, V., Vrba, F. J., Černis, K., Kazlauskas, A., Boyle, R. P., \& Davis hilip, A. G. 2007, Baltic Astron., 16, 349

Lonsdale, C., et al. 2003, PASP, 115, 897

Makovoz, D., \& Marleau, F. 2005, PASP, 117, 1113

Marcy, G. 1980, AJ, 85, 230

Megeath, S. T., et al. 2005, in IAU Symp. 227, Massive Star Birth: A Crossroads of Astrophysics, ed. R. Cesaroni et al. (Cambridge: Cambridge Univ. Press), 383

Menten, K. M., Reid, M. J., Forbrich, J., \& Brunthaler, A. 2007, A\&A, 474, 515

Merrill, P., \& Burwell, C. 1949, ApJ, 110, 387

Merrill, P., \& Burwell, C. 1950, ApJ, 112, 72

Miller, et al. 2011, ApJ, 730, 80

Moshir, M., Kopman, G., \& Conrow, T. 1992, IRAS Faint Source Survey and Explanatory Supplement (Pasadena, CA: IPAC)

Muench, A., Getman, K., Hillenbrand, L., \& Preibisch, T. 2008, in Handbook of Star Forming Regions, Vol. I: The Northern Sky, ed. B. Reipurth (ASP Monograph Publications, Vol. 4; San Francisco, CA: ASP), 483

Neckel, T., Harris, Q., \& Eiroa, C. 1980, A\&A, 92, L9

Ogura, K., Sugitani, K., \& Pickles, A. 2002, AJ, 123, 2597

Oliveira, I., et al. 2009, ApJ, 691, 672

Osterbrock, D. 1957, ApJ, 125, 622

Paradis, D., et al. 2010, A\&A, 520, 8

Price, S., Egan, M. P., Carey, S. J., Mizuno, D. R., \& Kuchar, T. A. 2001, AJ, 121, 2819 2001, AJ, 121, 2819

Rebull, L., et al. 2007, ApJS, 171, 447

Rebull, L., et al. 2010, ApJS, 186, 259

Reipurth, B., \& Schneider, N. 2008, in Handbook of Star Forming Regions, Vol. I: The Northern Sky, ed. B. Reipurth (ASP Monograph Publications, Vol. 4; San Francisco, CA: ASP), 36

Rieke, G., et al. 2004, ApJS, 154, 25

Robberto, M., Song, J., Mora Carrillo, G., Beckwith, S. V. W., Makidon, R. B., \& Panagia, N. 2004, ApJ, 606, 952

Robitaille, T., et al. 2008, AJ, 136, 2413

Shevchenko, V., Grankin, K. N., Ibragimov, M. A., Mel'Nikov, S. Yu., \& Yakubov, S. D. 1993, Ap\&SS, 202, 121

Skrutskie, M., et al. 2006, AJ, 131, 1163

Straižys, V., Corbally, C., \& Laugalys, V. 1999, Baltic Astron., 8, 355

Straižys, V., Corbally, C., \& Laugalys, V. 2008, Baltic Astron., 17, 125

Straižys, V., Kazlauskas, A., Vansevičius, V., \& Cernis, K. 1993, Baltic Astron., 2,171

Straižys, V., \& Laugalys, V. 2008a, Baltic Astron., 17, 1

Straižys, V., \& Laugalys, V. 2008b, Baltic Astron., 17, 143

Straižys, V., \& Laugalys, V. 2009, Baltic Astron., 18, 141

Straižys, V., Meištas, E., \& Vansevičius, V. 1989, Vilnius Observatory Bulletin, 83,3

Surace, J. A., et al. 2004, VizieR On-line Data Catalog II/255 (Pasadena, CA: Spitzer Science Center)

Terranegra, L., Chavarria-K, C., Diaz, S., \& Gonzalez-Patino, D. 1994, A\&AS, 104,557

Tielens, A. G. G. M. 2008, ARA\&A, 46, 289

Torres, R. M., Loinard, L., Mioduszewski, A. J., \& Rodríguez, L. F. 2007, ApJ, 671,1813

Villanova, S., Baume, G., Carraro, G., \& Geminale, A. 2004, A\&A, 419, 149

Welin, G. 1973, A\&AS, 9, 183

Wendker, H., Benz, D., \& Baars, J. 1983, A\&A, 124, 116

Werner, M., Uchida, K. I., Sellgren, K., Marengo, M., Gordon, K. D., Morris, P. W., Houck, J. R., \& Stansberry, J. A. 2004a, ApJS, 154, 309

Werner, M., et al. 2004b, ApJS, 154, 1

Wilking, B. A., Bontemps, S., Schuler, R. E., Greene, T. P., \& André, P. 2001, ApJ, 551, 357

Witham, A., Knigge, C., Drew, J. E., Greimel, R., Steeghs, D., Gänsicke, B. T., Groot, P. J., \& Mampaso, A. 2008, MNRAS, 384, 1277

Yamamura, I., et al. 2009, in ASP Conf. Ser. 418, AKARI, a Light to Illuminate the Misty Universe, ed. T. Onaka et al. (San Francisco, CA: ASP), 3

Zdanavičius, K., \& Straižys, V. 1990, Ap\&SS, 173, 309 